\title{
Detailed chemical composition of classical Cepheids in the LMC cluster NGC 1866 and in the field of the SMC ${ }^{\star}$
}

\author{
B. Lemasle ${ }^{1}$, M. A. T. Groenewegen ${ }^{2}$, E. K. Grebel ${ }^{1}$, G. Bono ${ }^{3,4}$, G. Fiorentino ${ }^{5}$, P. François ${ }^{6,7}$, L. Inno ${ }^{8}$, \\ V. V. Kovtyukh ${ }^{9,10}$, N. Matsunaga ${ }^{11}$, S. Pedicelli ${ }^{3,12}$, F. Primas ${ }^{12}$, J. Pritchard ${ }^{12}$, \\ M. Romaniello ${ }^{12,13}$, and R. da Silva ${ }^{3,4,14}$ \\ 1 Astronomisches Rechen-Institut, Zentrum für Astronomie der Universität Heidelberg, Mönchhofstr. 12-14, 69120 Heidelberg, \\ Germany \\ e-mail: lemasle@uni-heidelberg.de \\ 2 Koninklijke Sterrenwacht van België, Ringlaan 3, 1180 Brussels, Belgium \\ e-mail: martin.groenewegen@oma.be \\ 3 Dipartimento di Fisica, Università di Roma Tor Vergata, via della Ricerca Scientifica 1, 00133 Rome, Italy \\ ${ }^{4}$ INAF-Osservatorio Astronomico di Roma, via Frascati 33, 00078 Monte Porzio Catone, Rome, Italy \\ 5 INAF-Osservatorio Astronomico di Bologna, via Gobetti 93/3, 40129 Bologna, Italy \\ 6 GEPI, Observatoire de Paris, CNRS, Université Paris Diderot, place Jules Janssen, 92190 Meudon, France \\ 7 UPJV, Université de Picardie Jules Verne, 33 rue St. Leu, 80080 Amiens, France \\ 8 Max-Planck-Institut für Astronomie, 69117 Heidelberg, Germany \\ 9 Astronomical Observatory, Odessa National University, Shevchenko Park, 65014 Odessa, Ukraine \\ 10 Isaac Newton Institute of Chile, Odessa Branch, Shevchenko Park, 65014 Odessa, Ukraine \\ 11 Department of Astronomy, School of Science, The University of Tokyo, 7-3-1 Hongo, Bunkyo-ku, 113-0033 Tokyo, Japan \\ 12 European Southern Observatory, Karl-Schwarzschild-Str. 2, 85748 Garching bei München, Germany \\ 13 Excellence Cluster Universe, Boltzmannstr. 2, 85748 Garching bei München, Germany \\ 14 ASI Science Data Center, via del Politecnico snc, 00133 Rome, Italy
}

Received 14 June 2017 / Accepted 7 September 2017

\begin{abstract}
Context. Cepheids are excellent tracers of young stellar populations. They play a crucial role in astrophysics as standard candles. The chemistry of classical Cepheids in the Milky Way is now quite well-known, however despite a much larger sample, the chemical composition of Magellanic Cepheids has been only scarcely investigated.

Aims. For the first time, we study the chemical composition of several Cepheids located in the same populous cluster: NGC 1866, in the Large Magellanic Cloud (LMC). To also investigate the chemical composition of Cepheids at lower metallicity, we look at four targets located in the Small Magellanic Cloud (SMC). Our sample allows us to increase the number of Cepheids with known metallicities in the LMC/SMC by $20 \% / 25 \%$ and the number of Cepheids with detailed chemical composition in the LMC/SMC by $46 \% / 50 \%$.

Methods. We use canonical spectroscopic analysis to determine the chemical composition of Cepheids and provide abundances for a good number of $\alpha$, iron-peak, and neutron-capture elements.

Results. We find that six Cepheids in the LMC cluster NGC 1866 have a very homogeneous chemical composition, also consistent with red giant branch (RGB) stars in the cluster. Period-age relations that include no or average rotation indicate that all the Cepheids in NGC 1866 have a similar age and therefore belong to the same stellar population. Our results are in good agreement with theoretical models accounting for luminosity and radial velocity variations. Using distances based on period-luminosity relations in the near- or mid-infrared, we investigate for the first time the metallicity distribution of the young population in the SMC in the depth direction. Preliminary results show no metallicity gradient along the SMC main body, but our sample is small and does not contain Cepheids in the inner few degrees of the SMC.
\end{abstract}

Key words. stars: variables: Cepheids - Magellanic Clouds - galaxies: star clusters: individual: NGC 1866

\section{Introduction}

Classical Cepheids are the first step on the ladder of the extragalactic distance scale. Cepheid distances were first computed from period-luminosity (PL) relations in the optical bands, but the metallicity dependence of the optical PLrelations (e.g., Romaniello et al. 2008) and the interstellar

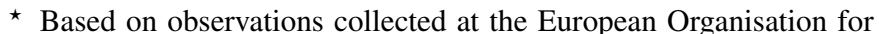
Astronomical Research in the Southern Hemisphere under ESO programme 082.D-0792(B).
}

absorption led researchers to prefer period-luminosity or periodWesenheit (PW) relations in the near-infrared (e.g., Bono et al. 2010; Feast et al. 2012; Ripepi et al. 2012; Gieren et al. 2013; Inno et al. 2013; Bhardwaj et al. 2016) where the Wesenheit index is a reddening-free quantity (Madore 1982). In recent years, these relations have been extended to the mid-infrared (e.g., Monson et al. 2012; Ngeow et al. 2012, 2015; Scowcroft et al. 2013; Rich et al. 2014); most of them are tied to very accurate parallax measurements for the closest Cepheids (Benedict et al. 2007; van Leeuwen et al. 2007). 
Independent distances to Cepheids can also be obtained with the Baade-Wesselink (BW) method, which combines the absolute variation of the radius of the star with the variation of its angular diameter. The former is obtained by integrating the pulsational velocity curve of the Cepheid that is derived from its radial velocity curve via the projection factor $(p)$. The latter uses surface-brightness (SB) relations to transform variations of the color of the Cepheid to variations of its angular diameter. SB relations were first derived in the optical bands (e.g., Wesselink 1969; Barnes \& Evans 1976) and extended to the near-infrared by Welch (1994), and Fouqué et al. (1997). Extremely accurate angular diameter variations can be obtained from interferometry (e.g., Mourard et al. 1997; Kervella et al. 2004) but this technique is currently limited to the closest Cepheids.

Published values of the $p$-factor consistently cluster around 1.3. However, the exact value of the $p$-factor and its dependence on the pulsation period remain uncertain at the level of 5-10\% (Kervella et al. 2017). In a series of papers, Storm et al. (2004a,b), Gieren et al. (2005), Fouqué et al. (2007) and Storm et al. $(2011 \mathrm{a}, \mathrm{b})$ found that the $p$-factor strongly depends on the period. Similar conclusions were obtained independently by Groenewegen et al. (2008, 2013). Using hydrostatic, spherically symmetric models of stellar atmospheres, Neilson et al. (2012) indicate that the $p$-factor varies with the period, but the dependence derived is not compatible with the observational results of, for example, Nardetto et al. (2014) and Storm et al. (2011a,b). To overcome these issues, Mérand et al. (2015) implemented a new flavor of the Baade-Wesselink method: they fit simultaneously all the photometric, interferometric, and radial velocity measurements in order to obtain a global model of the stellar pulsation. Applying this method to the Cepheids for which trigonometric parallaxes are available, Breitfelder et al. (2016) found a constant value of the $p$-factor, with no dependence on the pulsation period.

Among the aforementioned studies that include LMC/SMC Cepheids, only those of Groenewegen et al. (2008, 2013) rely on abundance determinations for individual Cepheids while the others use either the (oxygen) abundances derived in nearby HII regions or a mean, global abundance for a given galaxy. Because the determination of nebular abundances is still affected by uncertainties as pointed out by Kewley et al. (2008; but see, e.g., Pilyugin et al. 2016), and because the correlation between oxygen and iron varies from galaxy to galaxy, it is of crucial importance to have direct metallicity measurements in Cepheids. This task is now well achieved for Milky Way Cepheids (see Lemasle et al. 2007, 2008, 2013; Luck et al. 2011; Luck \& Lambert 2011; Genovali et al. 2013, 2014, 2015, and references therein). Despite the large number of Cepheids discovered in the Magellanic Clouds (3375/4630 in the LMC/SMC, respectively) by microlensing surveys such as OGLE (the Optical Gravitational Lensing Experiment; Udalski et al. 2015), only a few dozen have been followed up with high-resolution spectroscopy in order to determine their metallicities (Romaniello et al. 2005, 2008) or chemical composition (Luck \& Lambert 1992; Luck et al. 1998). In this context, it is worth mentioning that by transforming a hydrodynamical model of $\delta$ Cephei into a consistent model of the same star in the LMC, Nardetto et al. (2011) found a weak dependence of the $p$-factor on metallicity (1.5\% difference between LMC and solar metallicities).

NGC 1866 is of specific interest in that respect, as it is a young (age range of 100-200 Myr), massive cluster in the outskirts of the LMC that is known to harbor a large number (23) of Cepheids (e.g., Welch \& Stetson 1993). Many studies investigated the pulsational and evolutionary properties of the intermediate-mass stars in NGC 1866 (e.g., Bono et al. 1997; Fiorentino et al. 2007; Marconi et al. 2013; Musella et al. 2016) or the multiple stellar populations in LMC clusters (Milone et al. 2017). The focus on pulsating stars in NGC 1866 is obviously driven by the need to improve the extragalactic distance scale using either period-luminosity relations or the Baade-Wesselink methods (e.g., Storm et al. 2011a,b; Molinaro et al. 2012).

It is therefore quite surprising that the chemical composition of NGC 1866 stars has been investigated only in a few highresolution spectroscopic studies: Hill et al. (2000) analyzed a few elements in three red giant branch (RGB) stars in NGC 1866 and reported $[\mathrm{Fe} / \mathrm{H}]=-0.50 \pm 0.1 \mathrm{dex}$. Mucciarelli et al. (2011) derived the detailed chemical composition of 14 members of NGC 1866 and of 11 additional LMC field stars. They found an average $[\mathrm{Fe} / \mathrm{H}]=-0.43$ dex for NGC 1866. Colucci et al. (2011, 2012) determined the age and metallicity of NGC 1866 via high-resolution integrated-light spectroscopy and extended their work to other elements in Colucci \& Bernstein (2012). The study of Colucci et al. (2012) also includes three stellar targets in NGC 1866 for comparison purposes, with metallicities ranging from -0.31 to -0.39 dex.

In this paper, we focus on the chemical properties of six Cepheids in NGC 1866 and four field Cepheids in the SMC, and investigate what their chemical composition tells us about the stellar populations they belong to. Our sample increases the number of Cepheids with known metallicities in the LMC/SMC by $20 \% / 25 \%$ and the number of Cepheids with known detailed chemical composition in the LMC/SMC by $46 \% / 50 \%$. The Baade-Wesselink analysis will be presented in a companion paper.

\section{Observations}

We selected stars for which both optical \& near-infrared light curves and radial velocity measurements of good quality are already available, but for which no direct determination of the metallicity exists. We selected six Cepheids in the LMC NGC 1866 cluster and four field Cepheids in the SMC. The LMC cluster stars were observed with the FLAMES/UVES highresolution spectrograph (Pasquini et al. 2002) while the SMC field stars were observed with the UVES high-resolution spectrograph (Dekker et al. 2000). We used the red arm (CD \#3) standard template centered on $580 \mathrm{~nm}$ which offers a resolution of 47000 and covers the 476-684 nm wavelength range with a $5 \mathrm{~nm}$ gap around the central wavelength. We used the ESO reflex pipeline (Freudling et al. 2013) ${ }^{1}$ to perform the basic data reduction of the spectra. The heliocentric corrections of the radial velocities were computed with the IRAF task rvcorrect. The observing log is listed in Table 1. For the FLAMES/UVES sample, the weather conditions deteriorated during the night. We therefore analyzed only the first three spectra of a series of six for each star, as they reached a higher signal-to-noise ratio $(\mathrm{S} / \mathrm{N})$. The $\mathrm{S} / \mathrm{N}$ values are listed in Table 3 .

The phases were computed by adopting the period and the epoch of maximum light from OGLE IV (Udalski et al. 2015) as a zero point reference, except for HV 12202 for which no OGLE IV data are available. For this star, we used the values provided by Molinaro et al. (2012). The computations were made using

\footnotetext{
$1 \mathrm{ftp}$ ://ftp.eso.org/pub/dfs/pipelines/uves/ uves-fibre-pipeline-manual-18.8.1.pdf; ftp://ftp.eso.org/pub/dfs/pipelines/uves/ uves-pipeline-manual-22.14.1.pdf
} 
Table 1. Observing log.

\begin{tabular}{|c|c|c|c|c|}
\hline Target & Date & MJD & $\begin{array}{c}\text { Airmass } \\
\text { (start) }\end{array}$ & $\begin{array}{l}\text { Exp. time } \\
\text { (s) }\end{array}$ \\
\hline NGC 1866 & 2008-12-06Т00:35:23.385 & 54806.02457622 & 1.848 & 4800 \\
\hline NGC 1866 & 2008-12-06Т01:56:13.752 & 54806.08071473 & 1.541 & 4800 \\
\hline NGC 1866 & 2008-12-06Т03:17:03.638 & 54806.13684767 & 1.381 & 4800 \\
\hline NGC 1866 & 2008-12-06Т04:50:18.350 & 54806.20160128 & 1.320 & 4800 \\
\hline NGC 1866 & 2008-12-06Т06:11:08.646 & 54806.25773897 & 1.358 & 4800 \\
\hline NGC 1866 & 2008-12-06Т07:31:58.631 & 54806.31387305 & 1.490 & 3600 \\
\hline \multirow{3}{*}{ HV 822} & 2008-11-15Т00:57:58.745 & 54785.04026326 & 1.531 & 1000 \\
\hline & 2008-11-15T01:15:28.075 & 54785.05240828 & 1.523 & 1000 \\
\hline & 2008-11-15T01:32:57.446 & 54785.06455378 & 1.519 & 1000 \\
\hline \multirow[t]{3}{*}{ HV 1328} & 2008-11-15Т00:11:13.084 & 54785.00779033 & 1.569 & 800 \\
\hline & 2008-11-15T00:25:22.587 & 54785.01762254 & 1.556 & 800 \\
\hline & 2008-11-15Т00:39:31.570 & 54785.02744873 & 1.545 & 800 \\
\hline \multirow[t]{3}{*}{ HV 1333} & 2008-11-15T01:53:56.155 & 54785.07912217 & 1.531 & 1200 \\
\hline & 2008-11-15T02:14:45.620 & 54785.09358357 & 1.537 & 1200 \\
\hline & 2008-11-15T02:35:34.996 & 54785.10804394 & 1.547 & 1200 \\
\hline \multirow[t]{3}{*}{ HV 1335} & 2008-11-15T03:03:49.091 & 54785.12765152 & 1.569 & 1300 \\
\hline & 2008-11-15T03:26:18.517 & 54785.14326987 & 1.594 & 1300 \\
\hline & 2008-11-15T03:48:48.862 & 54785.15889887 & 1.625 & 1300 \\
\hline
\end{tabular}

Notes. The first six lines are spectra taken with the FLAMES/UVES multi-object spectrograph. The other spectra were taken with the UVES spectrograph.

heliocentric Julian dates (HJD), that is, 0.5 days were added to the modified Julian dates (MJD) and the light travel time between the Earth and the Sun was taken into account. The HJDs were double-checked using the IRAF task rvcorrect.

\section{Chemical abundances}

\subsection{Data analysis}

In our spectra, we measured the equivalent widths of the absorption lines with DAOSPEC (Stetson \& Pancino 2008): DAOSPEC fits lines with saturated Gaussians and all the lines detected are cross-correlated with a list of lines provided by the user. For each individual measurement of an equivalent width (EW), DAOSPEC provides the standard error, $\sigma_{\mathrm{EW}}$, on the measurement and a quality parameter, $Q$, that becomes higher in the regions where the quality of the spectrum decreases or for strong lines that deviate from a Gaussian profile. We selected only lines with $\sigma_{\mathrm{EW}} \leq 10 \%$ and $Q \leq 1.25$. For both the determination of the atmospheric parameters and the computation of the abundances, we considered only the lines with $20 \leq E W \leq 130 \mathrm{~m} \AA$.

The equivalent width method was favored as it enables a more homogeneous continuum placement, especially for spectra with a relatively low $\mathrm{S} / \mathrm{N}$ like ours (see examples in Fig. 1). The hyperfine structure can therefore not be taken into account. Current studies indicate that the effects of hyperfine structure splitting (hfs) are negligible or small for $\mathrm{Y}, \mathrm{Zr}, \mathrm{Nd}$, and $\mathrm{Eu}$ in Cepheids (da Silva et al. 2016), but not for Mn (Lemasle et al., in prep.) or to a lesser extent $\mathrm{La}$ (da Silva et al. 2016). A more detailed discussion about the hfs is provided in Sect. 3.6.

\subsection{Radial velocities}

For the NGC 1866 sample, the accuracy of the radial velocity determined by DAOSPEC is in general better than $\pm 2 \mathrm{~km} \mathrm{~s}^{-1}$, with a mean error in the individual velocities measurement of

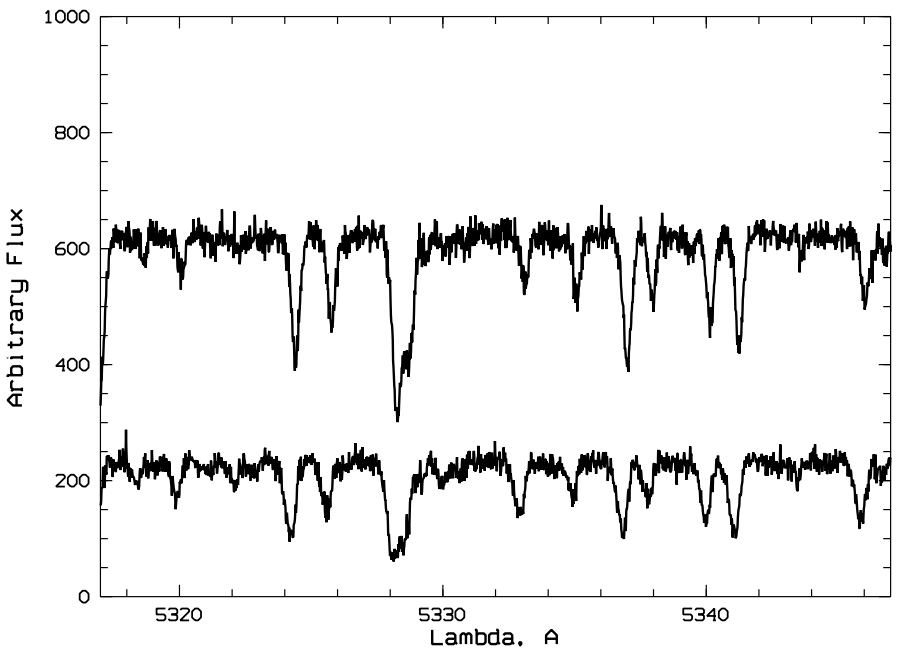

Fig. 1. Excerpts of spectra covering the 5317-5347 $\AA$ range. Top: HV $1328($ SMC) at MJD $=54785.01762254(S / N \approx 20)$. Bottom: HV 12198 (NGC 1866) at $54806.13684767(S / N \approx 30)$.

$1.157 \mathrm{~km} \mathrm{~s}^{-1}$. Thanks to a higher $\mathrm{S} / \mathrm{N}$, the radial velocities for the SMC sample are even more accurate, with a mean error of $0.804 \mathrm{~km} \mathrm{~s}^{-1}$. Our measurements are listed in Table 2 . Comments in footnotes come from the OGLE-III database (Soszyński et al. 2010). We note that in both cases the radial velocities obtained from the lower (L) and the upper (U) chip of the UVES red arm are in excellent agreement. The averaged radial velocities and the heliocentric corrections (computed with the IRAF task rvcorrect, with a negligible uncertainty of $\approx 0.005 \mathrm{~km} \mathrm{~s}^{-1}$ ) are also listed in Table 2 .

Because this was one of our target selection criteria, there is an extensive amount of radial velocity data available for the Cepheids in our sample. From these data it was possible to ascertain that our NGC 1866 Cepheids are indeed cluster mem- 
Table 2. Radial velocities for our targets in the LMC cluster NGC 1866 and in the field of the SMC.

\begin{tabular}{|c|c|c|c|c|c|c|c|}
\hline \multicolumn{8}{|c|}{ Targets in the LMC cluster NGC 1866} \\
\hline Target & $\begin{array}{l}\text { Period }(P) \\
\quad(\mathrm{d})\end{array}$ & Phase $\phi$ & $\begin{array}{c}\operatorname{Vr}_{\mathrm{L}}^{a} \\
\left(\mathrm{~km} \mathrm{~s}^{-1}\right)\end{array}$ & $\begin{array}{c}\mathrm{Vr}_{\mathrm{U}}^{b} \\
\left(\mathrm{~km} \mathrm{~s}^{-1}\right)\end{array}$ & $\begin{array}{c}\mathrm{Vr} \text { (averaged) } \\
\left(\mathrm{km} \mathrm{s}^{-1}\right)\end{array}$ & $\begin{array}{l}\text { Heliocentric correction } \\
\left(\mathrm{km} \mathrm{s}^{-1}\right)\end{array}$ & $\begin{array}{l}\text { Vr corrected } \\
\left(\mathrm{km} \mathrm{s}^{-1}\right)\end{array}$ \\
\hline \multirow[t]{3}{*}{ HV 12197} & 3.1437642 & 0.081 & $283.399 \pm 1.919$ & $282.260 \pm 3.202$ & $283.098 \pm 1.646$ & -2.205 & $280.893 \pm 1.646$ \\
\hline & & 0.099 & $284.238 \pm 1.390$ & $284.180 \pm 1.641$ & $284.214 \pm 1.061$ & -2.242 & $281.972 \pm 1.061$ \\
\hline & & 0.117 & $285.289 \pm 1.567$ & $284.997 \pm 1.378$ & $285.124 \pm 1.035$ & -2.294 & $282.830 \pm 1.035$ \\
\hline \multirow[t]{3}{*}{ HV 12198} & 3.5227781 & 0.643 & $315.940 \pm 1.384$ & $315.921 \pm 1.552$ & $315.932 \pm 1.033$ & -2.190 & $313.742 \pm 1.033$ \\
\hline & & 0.659 & $316.662 \pm 1.031$ & $316.566 \pm 1.332$ & $316.626 \pm 0.815$ & -2.227 & $314.399 \pm 0.815$ \\
\hline & & 0.675 & $316.960 \pm 1.305$ & $316.943 \pm 1.097$ & $316.950 \pm 0.840$ & -2.279 & $314.671 \pm 0.840$ \\
\hline \multirow[t]{3}{*}{ HV 12199} & 2.6391571 & 0.928 & $289.626 \pm 2.129$ & $289.346 \pm 4.279$ & $289.570 \pm 1.906$ & -2.199 & $287.371 \pm 1.906$ \\
\hline & & 0.949 & $284.720 \pm 1.187$ & $284.655 \pm 2.177$ & $284.705 \pm 1.042$ & -2.236 & $282.469 \pm 1.042$ \\
\hline & & 0.970 & $281.507 \pm 1.528$ & $281.312 \pm 1.867$ & $281.429 \pm 1.182$ & -2.288 & $279.141 \pm 1.182$ \\
\hline \multirow[t]{3}{*}{ HV 12202} & 3.101207 & 0.807 & $319.318 \pm 2.632$ & $318.444 \pm 4.291$ & $319.079 \pm 2.244$ & -2.180 & $316.899 \pm 2.244$ \\
\hline & & 0.825 & $316.907 \pm 2.040$ & $316.018 \pm 2.053$ & $316.465 \pm 1.447$ & -2.217 & $314.248 \pm 1.447$ \\
\hline & & 0.843 & $313.635 \pm 1.472$ & $312.713 \pm 3.442$ & $313.492 \pm 1.353$ & -2.269 & $311.223 \pm 1.353$ \\
\hline \multirow[t]{3}{*}{ HV 12203} & 2.9541342 & 0.765 & $323.664 \pm 1.902$ & $323.307 \pm 2.098$ & $323.503 \pm 1.409$ & -2.180 & $321.323 \pm 1.409$ \\
\hline & & 0.784 & $322.905 \pm 1.775$ & $322.400 \pm 1.448$ & $322.602 \pm 1.122$ & -2.217 & $320.385 \pm 1.122$ \\
\hline & & 0.803 & $320.983 \pm 1.407$ & $321.260 \pm 1.702$ & $321.095 \pm 1.084$ & -2.269 & $318.826 \pm 1.084$ \\
\hline \multirow[t]{3}{*}{ HV 12204} & 3.4387315 & 0.519 & $292.454 \pm 0.524$ & $292.107 \pm 0.955$ & $292.374 \pm 0.459$ & -2.163 & $290.211 \pm 0.459$ \\
\hline & & 0.535 & $293.664 \pm 0.919$ & $293.164 \pm 0.751$ & $293.364 \pm 0.582$ & -2.200 & $291.164 \pm 0.582$ \\
\hline & & 0.551 & $294.177 \pm 0.713$ & $294.166 \pm 0.895$ & $294.173 \pm 0.558$ & -2.252 & $291.921 \pm 0.558$ \\
\hline \multicolumn{8}{|c|}{ Targets in the SMC } \\
\hline \multirow[t]{3}{*}{$\mathrm{HV} 822^{c}$} & 16.7419693 & 0.998 & $101.288 \pm 1.736$ & $101.438 \pm 2.309$ & $101.342 \pm 1.388$ & -12.692 & $88.650 \pm 1.388$ \\
\hline & & 0.999 & $101.143 \pm 1.931$ & $101.027 \pm 1.672$ & $101.077 \pm 1.264$ & -12.701 & $88.376 \pm 1.264$ \\
\hline & & 0.999 & $101.450 \pm 0.939$ & $100.845 \pm 1.543$ & $101.286 \pm 0.802$ & -12.709 & $88.577 \pm 0.802$ \\
\hline \multirow[t]{3}{*}{ HV $1328^{d}$} & 15.8377104 & 0.883 & $121.800 \pm 1.013$ & $121.771 \pm 1.605$ & $121.792 \pm 0.857$ & -12.808 & $108.984 \pm 0.857$ \\
\hline & & 0.884 & $121.592 \pm 0.626$ & $121.509 \pm 0.615$ & $121.550 \pm 0.439$ & -12.814 & $108.736 \pm 0.439$ \\
\hline & & 0.884 & $121.402 \pm 1.069$ & $121.716 \pm 0.876$ & $121.590 \pm 0.678$ & -12.821 & $108.769 \pm 0.678$ \\
\hline \multirow[t]{3}{*}{ HV 1333} & 16.2961015 & 0.659 & $179.553 \pm 1.333$ & $179.786 \pm 0.735$ & $179.732 \pm 0.644$ & -12.742 & $166.990 \pm 0.644$ \\
\hline & & 0.660 & $179.816 \pm 1.267$ & $179.909 \pm 0.930$ & $179.876 \pm 0.750$ & -12.752 & $167.124 \pm 0.750$ \\
\hline & & 0.661 & $179.486 \pm 1.555$ & $179.972 \pm 0.827$ & $179.865 \pm 0.730$ & -12.761 & $167.104 \pm 0.730$ \\
\hline \multirow[t]{3}{*}{ HV 1335} & 14.3813503 & 0.318 & $163.310 \pm 0.763$ & $163.376 \pm 1.360$ & $163.326 \pm 0.665$ & -12.750 & $150.576 \pm 0.665$ \\
\hline & & 0.319 & $162.985 \pm 1.154$ & $163.194 \pm 1.102$ & $163.094 \pm 0.797$ & -12.760 & $150.334 \pm 0.797$ \\
\hline & & 0.320 & $163.352 \pm 1.466$ & $163.198 \pm 0.877$ & $163.239 \pm 0.753$ & -12.769 & $150.470 \pm 0.753$ \\
\hline
\end{tabular}

Notes. The radial velocities derived for the lower (L) and upper (U) chips of the UVES red arm are listed in Cols. 4 and 5. The averaged values are listed in Col. 6, the barycentric corrections in Col. 7 and the final values for the radial velocity (after correction) in Col. 8. ${ }^{(a)}$ Red arm lower chip. ${ }^{(b)}$ Red arm upper chip. ${ }^{(c)}$ Secondary period of 1.28783 d (OGLE-III database). ${ }^{(d)}$ Secondary period of $14.186 \mathrm{~d}$ (OGLE-III database).

bers. Excluding variable stars, Mucciarelli et al. (2011) report an average heliocentric velocity of $v=298.5 \pm 0.4 \mathrm{~km} \mathrm{~s}^{-1}$ with a dispersion of $\sigma=1.6 \mathrm{~km} \mathrm{~s}^{-1}$. For both the LMC and SMC targets, our radial velocity measurements are in excellent agreement with the expected values at the given pulsation phase obtained from the radial velocity curves published in the literature (Welch et al. 1991; Storm et al. 2004a, 2005; Molinaro et al. 2012; Marconi et al. 2013, 2017).

Systematic shifts between different samples are generally attributed to the orbital motion in a binary system. Two stars in our sample (HV 12202 and HV 12204) were identified as spectroscopic binaries (Welch et al. 1991; Storm et al. 2005). As far as HV 12202 is concerned, our measurements are in good agreement with all the data compiled by Storm et al. (2005) except for their CTIO data and the latest part of the Welch et al. (1991) data, and therefore support the shifts of $+18 \mathrm{~km} \mathrm{~s}^{-1}$ (respectively $+21 \mathrm{~km} \mathrm{~s}^{-1}$ ) applied to these datasets in order to provide a homogeneous radial velocity curve. For the same purpose, the latest data from Welch et al. (1991) had to be shifted by $+7 \mathrm{~km} \mathrm{~s}^{-1}$ and our measurements should be shifted by $\approx+15 \mathrm{~km} \mathrm{~s}^{-1}$ in the case of HV 12204 . Binarity is a common feature for Milky Way Cepheids (more than $50 \%$ of them are binaries, see Szabados 2003), but there is a strong observational bias with distance and indeed the number of known binaries is much lower for the farther, fainter Cepheids in the Magellanic Clouds (Szabados \& Nehéz 2012). It should be noted that Anderson (2014) found modulations in the radial velocity curves of four Galactic Cepheids. However, the order of magnitude of the effect ranges from several hundred $\mathrm{m} \mathrm{s}^{-1}$ to a few $\mathrm{km} \mathrm{s}^{-1}$ and cannot account for the differences reported here in the case of HV 12202 and HV 12204.

\subsection{Atmospheric parameters}

As Cepheids are variable stars, simultaneous photometric and spectroscopic observations are in general not available and the atmospheric parameters are usually derived from the spectra only. Kovtyukh \& Gorlova (2000) have developed an accurate method to derive the effective temperature $T_{\text {eff }}$ from the depth ratio of carefully chosen pairs of lines that have been used extensively in Cepheids studies (Andrievsky et al. 2002a; Luck \& Lambert 2011). 
B. Lemasle et al.: Detailed chemical composition of classical Cepheids in the Magellanic Clouds

Table 3. Coordinates, properties and atmospheric parameters for the Cepheids in our sample.

\begin{tabular}{|c|c|c|c|c|c|c|c|c|c|c|c|}
\hline \multicolumn{12}{|c|}{ Targets in the LMC cluster NGC 1866} \\
\hline Cepheid & $\begin{array}{l}\text { RA (J2000) } \\
\quad(\mathrm{dms})\end{array}$ & $\begin{array}{c}\text { Dec }(\mathrm{J} 2000) \\
(\mathrm{dms})\end{array}$ & $\begin{array}{c}V \\
(\mathrm{mag})\end{array}$ & $\begin{array}{c}P \\
(d) \\
\end{array}$ & $\phi$ & $\begin{array}{c}T_{\text {eff }}(\mathrm{LDR}) \\
(\mathrm{K})\end{array}$ & $\begin{array}{l}T_{\text {eff }} \\
(\mathrm{K})\end{array}$ & $\begin{array}{l}\log g \\
(\operatorname{dex})\end{array}$ & $\begin{array}{c}V_{t} \\
\left(\mathrm{~km} \mathrm{~s}^{-1}\right) \\
\end{array}$ & $\begin{array}{c}{[\mathrm{Fe} / \mathrm{H}]} \\
(\mathrm{dex})\end{array}$ & $\begin{array}{c}S / N \\
(5228 / 5928 \AA) \\
\end{array}$ \\
\hline \multirow[t]{3}{*}{ HV 12197} & 051313.0 & -653048 & 16.116 & 3.1437642 & 0.081 & $6060 \pm 97(3)$ & 6150 & 1.5 & 3.1 & -0.35 & $16 / 15$ \\
\hline & & & & 3.1437642 & 0.099 & & 6150 & 1.5 & 3.2 & -0.35 & $28 / 27$ \\
\hline & & & & 3.1437642 & 0.117 & & 6100 & 1.5 & 3.1 & -0.35 & $27 / 25$ \\
\hline \multirow[t]{3}{*}{ HV 12198} & 051326.7 & -652705 & 15.970 & 3.5227781 & 0.643 & $5634 \pm 85(6)$ & 5625 & 1.4 & 3.4 & -0.35 & $13 / 19$ \\
\hline & & & & 3.5227781 & 0.659 & & 5625 & 1.5 & 3.6 & -0.35 & $20 / 26$ \\
\hline & & & & 3.5227781 & 0.675 & & 5625 & 1.4 & 3.6 & -0.35 & $21 / 23$ \\
\hline \multirow[t]{3}{*}{ HV 12199} & 051319.0 & -652930 & 16.283 & 2.6391571 & 0.928 & - & 6550 & 2.2 & 3.2 & -0.30 & $15 / 14$ \\
\hline & & & & 2.6391571 & 0.949 & & 6600 & 2.1 & 3.0 & -0.30 & $29 / 31$ \\
\hline & & & & 2.6391571 & 0.970 & & 6650 & 2.0 & 3.1 & -0.35 & $26 / 32$ \\
\hline \multirow[t]{3}{*}{ HV 12202} & 051339.0 & -652900 & 16.08 & 3.101207 & 0.807 & $5712 \pm 100(6)$ & 5775 & 1.6 & 3.1 & -0.40 & $17 / 14$ \\
\hline & & & & 3.101207 & 0.825 & & 5900 & 1.6 & 3.1 & -0.40 & $20 / 25$ \\
\hline & & & & 3.101207 & 0.843 & & 5900 & 1.5 & 2.9 & -0.40 & $20 / 24$ \\
\hline \multirow[t]{3}{*}{ HV 12203} & 051340.0 & -652936 & 16.146 & 2.9541342 & 0.765 & $5856 \pm 117(9)$ & 5850 & 1.7 & 3.5 & -0.35 & $16 / 19$ \\
\hline & & & & 2.9541342 & 0.784 & & 5800 & 1.2 & 3.3 & -0.35 & $17 / 26$ \\
\hline & & & & 2.9541342 & 0.803 & & 5800 & 1.6 & 3.4 & -0.35 & $19 / 24$ \\
\hline \multirow[t]{3}{*}{ HV 12204} & 051358.0 & -652848 & 15.715 & 3.4387315 & 0.519 & $5727 \pm 98(11)$ & 5700 & 1.2 & 2.8 & -0.35 & $19 / 23$ \\
\hline & & & & 3.4387315 & 0.535 & & 5725 & 1.3 & 2.9 & -0.35 & $22 / 31$ \\
\hline & & & & 3.4387315 & 0.551 & & 5700 & 1.2 & 2.9 & -0.35 & $21 / 28$ \\
\hline \multicolumn{12}{|c|}{ Targets in the SMC } \\
\hline \multirow[t]{3}{*}{ HV 822} & 004155.5 & -733223 & 14.524 & 16.7419693 & 0.998 & - & 6400 & 1.8 & 2.7 & -0.75 & $33 / 48$ \\
\hline & & & & 16.7419693 & 0.999 & & 6400 & 1.8 & 2.7 & -0.75 & $41 / 46$ \\
\hline & & & & 16.7419693 & 0.999 & & 6400 & 1.8 & 2.7 & -0.75 & $35 / 42$ \\
\hline \multirow[t]{3}{*}{ HV 1328} & 003254.9 & -734919 & 14.115 & 15.8377104 & 0.883 & $6325 \pm 98(5)$ & 6100 & 1.9 & 2.6 & -0.60 & $26 / 37$ \\
\hline & & & & 15.8377104 & 0.884 & & 6100 & 1.9 & 2.6 & -0.60 & $31 / 37$ \\
\hline & & & & 15.8377104 & 0.884 & & 6100 & 1.9 & 2.6 & -0.60 & $29 / 40$ \\
\hline \multirow[t]{3}{*}{ HV 1333} & 003603.5 & -735558 & 14.729 & 16.2961015 & 0.659 & $5192 \pm 102(8)$ & 5175 & 0.4 & 3.2 & -0.90 & $18 / 26$ \\
\hline & & & & 16.2961015 & 0.660 & & 5200 & 0.4 & 2.8 & -0.80 & $19 / 25$ \\
\hline & & & & 16.2961015 & 0.661 & & 5175 & 0.4 & 3.2 & -0.90 & $16 / 30$ \\
\hline \multirow[t]{3}{*}{ HV 1335} & 003655.7 & -735628 & 14.762 & 14.3813503 & 0.318 & $5566 \pm 156(6)$ & 5600 & 0.6 & 2.6 & -0.80 & $25 / 29$ \\
\hline & & & & 14.3813503 & 0.319 & & 5675 & 0.8 & 2.6 & -0.75 & $28 / 29$ \\
\hline & & & & 14.3813503 & 0.320 & & 5600 & 0.6 & 2.7 & -0.80 & $25 / 29$ \\
\hline
\end{tabular}

Notes. $V$ magnitudes and periods are from OGLE IV, except for HV 12202, for which they have been found in Molinaro et al. (2012) and Musella et al. (2016). Column 7 refers to the $T_{\text {eff }}$ derived from the line depth ratio method (LDR, Kovtyukh \& Gorlova 2000) while Col. 8 is the $T_{\text {eff }}$ derived from the excitation equilibrium. The last column lists the $\mathrm{S} / \mathrm{N}$ around 5228 and $5928 \AA$, respectively.

As the red CCD detector of UVES is made of two chips side by side (lower: $\mathrm{L}$; upper: $\mathrm{U}$ ), there is a gap of $\approx 50 \AA$ around the central wavelength ( $580 \mathrm{~nm}$ in our case) and we could not use the lines falling in this spectral domain. Moreover, the line depth ratios have been calibrated for Milky Way Cepheids that are more metal-rich than the Magellanic Cepheids (especially in the case of the SMC) $)^{2}$. It also turns out that several stars in our sample were observed at a phase where they reach higher $T_{\text {eff }}(>6000 \mathrm{~K})$ during the pulsation cycle. The combination of a high $T_{\text {eff }}$ and a rather low metallicity made it very challenging to measure the depth of some lines, and in particular the weak line of the pairs. As a result, we could only use a limited number of line depth ratios (typically 5-10 out of 32) to determine $T_{\text {eff }}$. Moreover, for two stars (HV 12199 and HV 822), we were unable to determine $T_{\text {eff }}$ from the line depth ratio as their temperature $(>6400 \mathrm{~K})$ at

2 The metallicity of Milky Way Cepheids continuously decreases from $+0.4-+0.5$ dex in the inner disk (e.g., Andrievsky et al. 2002b; Pedicelli et al. 2010; Martin et al. 2015; Andrievsky et al. 2016) to $\approx-0.4$ dex in the outer disk (e.g., Luck et al. 2003; Lemasle et al. 2008). Current high-resolution spectroscopic studies indicate that Cepheids have metallicities ranging from -0.62 to -0.10 dex (Luck \& Lambert 1992; Luck et al. 1998; Romaniello et al. 2008) in the LMC and from -0.87 to -0.63 dex in the SMC. the time of the observations fell above the range of temperatures where most ratios are calibrated ${ }^{3}$.

To ensure the determination of $T_{\text {eff }}$, we double-checked that lines with both high and low $\chi_{\mathrm{ex}}$ values properly fit the curve of growth (see Appendix D) and that the Fe I abundances are independent from the excitation potential of the lines. In a canonical spectroscopic analysis, we determined the surface gravity $\log g$ and the microturbulent velocity $V_{t}$ by imposing that the ionization balance between Fe I and Fe II is satisfied and that the Fe I abundance is independent from the EW of the lines. On average we have at our disposal $42 \mathrm{Fe} \mathrm{I} / 7 \mathrm{Fe}$ II lines in the NGC 1866 Cepheids and $42 \mathrm{Fe} \mathrm{I/11} \mathrm{Fe} \mathrm{II} \mathrm{lines} \mathrm{in} \mathrm{the} \mathrm{SMC} \mathrm{Cepheids.} \mathrm{We}$ note that the adopted $T_{\text {eff }}$ values are in general in very good agreement with those derived from the line depth ratios. The atmospheric parameters are listed in Table 3.

As mentioned above, the Cepheids in our sample have rather high temperatures, two of them hot enough at the phase of the observations to prevent the use of line depth ratios to determine their temperature. It has been noted before (Brocato et al. 2004) that these stars are located in the color-magnitude diagram at the hot tip of the so-called "blue nose" experienced by

3 Depending on the ratio, the upper limit varies between 6200 and $6700 \mathrm{~K}$. 
core He-burning supergiants. During this evolutionary stage they cross the instability strip and start pulsating.

As we impose the ionization balance between Fe I and Fe II to derive $\log g$, NLTE effects affecting primarily Fe I could hamper an accurate determination of $\log g$ (Luck \& Lambert 1985). There is currently no extensive study of NLTE effects in Cepheids, although NLTE abundances have been derived for some individual elements like O (Korotin et al. 2014, and references therein) or Ba (Andrievsky et al. 2014, and references therein). It is beyond the scope of this paper to provide a full discussion of NLTE effects in Cepheids, and we refer the reader to the discussion in, for example, Kovtyukh \& Andrievsky (1999) or Yong et al. (2006). Several arguments have been brought forward to support the fact that NLTE effects may be limited in Cepheids. For instance, Andrievsky et al. (2005) followed several Cepheids with $3 \mathrm{~d}<P<6 \mathrm{~d}$ throughout the entire period and found identical $[\mathrm{Fe} / \mathrm{H}]$ and abundances ratios (within the uncertainties), although $T_{\text {eff }}$ varies by $\approx 1000 \mathrm{~K}$ (the same holds for Cepheids with different period ranges studied in this series of papers). Also Yong et al. (2006) found a mean difference $[\mathrm{TiI} / \mathrm{Fe}]-[\mathrm{TiII} / \mathrm{Fe}]=0.07 \pm 0.02(\sigma=0.11)$. As this difference falls within the measurement uncertainties, they concluded that the values of $\log g$ obtained via the ionization equilibrium of FeI/FeII are satisfactory. All these arguments point toward the fact that a canonical spectroscopic analysis provides consistent, reliable results. However, the aforementioned studies deal with Milky Way Cepheids. As Magellanic Cepheids are slightly more metal-poor, NLTE effects should be slightly more pronounced than in the Galactic ones. In a study of nine LMC F supergiants, Hill et al. (1995) introduced an overionization law and obtained higher (+0.6 dex) spectroscopic gravities that are in good agreement with those derived from photometry. They note that $[\mathrm{Fe} / \mathrm{H}]$ becomes only +0.1 dex higher than in the LTE case and that the global abundance pattern remains unchanged, as already reported by, for example, Spite et al. (1989).

\subsection{Comparison with models}

Marconi et al. (2013) used non-linear convective pulsation models in order to reproduce simultaneously the light curves in several photometric bands and the radial velocity curves of a few Cepheids in NGC 1866. For HV 12197 they reached good agreement between theory and observations and report a mean $T_{\text {eff }}$ of $5850 \mathrm{~K}$. They also plotted the temperature predicted by the model and for the phases $0.08-0.12$ they found $T_{\text {eff }}$ of the order of $6300 \mathrm{~K}$ and slightly below (their Fig. 8, bottom panel), in quite good agreement with our measurements that fall around $6150 \mathrm{~K}$. For HV 12199 , they report a mean $T_{\text {eff }}$ of $6125 \mathrm{~K}$ but had to modify notably the projection factor to reach the best match with the radial velocity curve. They also mention that using the light curves would only lead to a hotter star $\left(\left\langle T_{\text {eff }}\right\rangle=6200 \mathrm{~K}\right)$, but in this case an even lower (and unrealistic) value would be required for the projection factor in order to fit the radial velocity curve. The $T_{\text {eff }}$ curve for HV 12199 (their Fig. 8, top panel) in the phases $0.93-0.97$ shows a rapid rise of the temperature and the corresponding $T_{\text {eff }}$ value of $\approx 6250-6300 \mathrm{~K}$, somewhat below the values around $6600 \mathrm{~K}$ we determined for $T_{\text {eff }}$.

\subsection{Abundance determinations}

Our abundance analysis is based on equivalent widths measured with DAOSPEC (see Sect. 3.1). We derived the abundances of 16 elements (several of them in two ionization states) for which absorption lines could be measured in the spectral domain covered by the UVES red arm (CD \#3, $580 \mathrm{~nm}$ ) standard template. In a few cases we updated the linelists of Genovali et al. (2013) and Lemasle et al. (2013) with oscillator strengths and excitation potentials from recent releases of the Vienna Atomic Lines Database (VALD, Kupka et al. 1999, and references therein) and from the Gaia-ESO survey linelist (Heiter et al. 2015). We took the values tabulated by Anders \& Grevesse (1989) as solar references, except for Fe and Ti for which we used $\log \epsilon_{\mathrm{Fe}}=7.48$ and $\log \epsilon_{\mathrm{Ti}}=5.02$. We used MARCS (1D LTE spherical) atmosphere models (Gustafsson et al. 2008) covering the parameter space of Magellanic Clouds Cepheids. Abundances were computed with calrai, a LTE spectrum synthesis code originally developed by Spite (1967) and continuously updated since then. For a given element, the abundance derived from a single spectrum is estimated as the mean value of the abundances determined for each individual line of this element. The final abundance of a star is then obtained by computing the weighted mean (and standard deviation) for the three spectra analyzed, where the weight is the number of lines of a given element measured in each spectrum.

\subsection{Abundances}

We provide the abundances of one light element $(\mathrm{Na})$, several $\alpha$-elements ( $\mathrm{Mg}, \mathrm{Si}, \mathrm{S}, \mathrm{Ca}, \mathrm{Ti}$ ), iron-peak elements ( $\mathrm{Sc}, \mathrm{Cr}, \mathrm{Mn}$, $\mathrm{Fe}, \mathrm{Ni}$ ), and neutron capture elements (Y, Zr, La, Nd, Eu). As already mentioned, we analyzed three individual (back to back) spectra for each star and the abundances derived are in most cases in excellent agreement. As expected, the size of the error bars is correlated to the number of lines analyzed. In contrast to our Cepheid studies in the Milky Way, where Si comes second after iron for the number of lines measured, the UVES red arm (CD \#3, $580 \mathrm{~nm}$ ) spectral domain contains only a few Si lines with sufficient quality but a larger number of $\mathrm{Ca}$ lines and indeed 9-11 calcium lines were usually measured in our spectra. The individual abundances (per spectrum) are listed in Tables A.1-A.6 for the NGC 1866 Cepheids and in Tables B.1-B.4 for the SMC Cepheids. The last two columns of these tables list the weighted means and standard deviations adopted as the chemical composition of the star in the remainder of the paper.

Molinaro et al. (2012) provide the metallicities for three Cepheids in NGC 1866, analyzed in the same way as the stars in Mucciarelli et al. (2011). Two of Molinaro et al. (2012) Cepheids are also included in our sample, namely HV 12197 and HV 12199. Taking into account a tiny difference (0.02 dex) in the solar reference value for $[\mathrm{Fe} / \mathrm{H}]$, the results agree very well; they report $[\mathrm{Fe} / \mathrm{H}]=-0.39 \pm 0.05$ for $\mathrm{HV} 12197$, while we found $-0.33 \pm 0.07 \mathrm{dex}$, and $[\mathrm{Fe} / \mathrm{H}]=-0.38 \pm 0.06$ for HV 12199, while we found $-0.31 \pm 0.05$.

For a good number of our spectra, several elements ( $\mathrm{Si}, \mathrm{Ti}$, $\mathrm{Cr}$ ) could be measured in two ionization states, in addition to the usual Fe I/Fe II. When the ionization equilibrium is reached for iron, it is usually also reached for the other elements as the abundances derived from the neutral and ionized species agree within the error bars, thus reinforcing our confidence in our atmospheric parameters, in particular log $g$. In order to quantify how the results are affected by uncertainties in the atmospheric parameters, we computed the abundances with over- or underestimated values of $T_{\text {eff }}( \pm 150 \mathrm{~K}), \log g( \pm 0.3 \mathrm{dex}), V_{t}\left( \pm 0.5 \mathrm{~km} \mathrm{~s}^{-1}\right)$ for two spectra at different $T_{\text {eff. }}$. Uncertainties in $[\mathrm{Fe} / \mathrm{H}]$ leave the abundances unchanged and are therefore not considered in this exercise. The sum in quadrature of the differences in the computed abundances is adopted as the uncertainty in the abundances due 
Table 4. Uncertainties in the final abundances due to uncertainties in the atmospheric parameters.

\begin{tabular}{|c|c|c|c|c|}
\hline \multicolumn{5}{|c|}{ Error budget for HV 12198, $\phi=0.675(\mathrm{MJD}=54806.13684767)$} \\
\hline Element & $\begin{array}{c}\Delta T_{\text {eff }} \\
( \pm 150 \mathrm{~K}) \\
(\mathrm{dex})\end{array}$ & $\begin{array}{c}\Delta \log g \\
( \pm 0.3 \mathrm{dex}) \\
(\mathrm{dex})\end{array}$ & $\begin{array}{c}\Delta V_{t} \\
\left( \pm 0.5 \mathrm{~km} \mathrm{~s}^{-1}\right) \\
(\mathrm{dex})\end{array}$ & $\begin{array}{c}\text { Quadratic } \\
\text { sum } \\
(\text { dex })\end{array}$ \\
\hline$[\mathrm{NaI} / \mathrm{H}]$ & 0.08 & 0.00 & 0.03 & 0.09 \\
\hline$[\mathrm{MgI} / \mathrm{H}]$ & 0.14 & 0.00 & 0.08 & 0.16 \\
\hline$[\mathrm{SiI} / \mathrm{H}]$ & 0.07 & 0.01 & 0.03 & 0.07 \\
\hline$[\mathrm{SiII} / \mathrm{H}]$ & 0.12 & 0.12 & 0.11 & 0.19 \\
\hline$[\mathrm{SI} / \mathrm{H}]$ & 0.07 & 0.08 & 0.04 & 0.11 \\
\hline$[\mathrm{CaI} / \mathrm{H}]$ & 0.10 & 0.00 & 0.06 & 0.12 \\
\hline$[\mathrm{ScII} / \mathrm{H}]$ & 0.03 & 0.12 & 0.08 & 0.15 \\
\hline$[\mathrm{TiI} / \mathrm{H}]$ & 0.15 & 0.00 & 0.03 & 0.15 \\
\hline$[\mathrm{TiII} / \mathrm{H}]$ & 0.02 & 0.12 & 0.02 & 0.12 \\
\hline$[\mathrm{CrII} / \mathrm{H}]$ & 0.02 & 0.11 & 0.07 & 0.13 \\
\hline$[\mathrm{MnI} / \mathrm{H}]$ & 0.12 & 0.00 & 0.03 & 0.12 \\
\hline$[\mathrm{FeI} / \mathrm{H}]$ & 0.13 & 0.00 & 0.05 & 0.13 \\
\hline$[\mathrm{FeII} / \mathrm{H}]$ & 0.01 & 0.12 & 0.05 & 0.13 \\
\hline$[\mathrm{NiI} / \mathrm{H}]$ & 0.13 & 0.00 & 0.04 & 0.13 \\
\hline [YII/H] & 0.04 & 0.12 & 0.05 & 0.13 \\
\hline$[\mathrm{ZrII} / \mathrm{H}]$ & 0.03 & 0.11 & 0.03 & 0.12 \\
\hline$[\mathrm{LaII} / \mathrm{H}]$ & 0.07 & 0.12 & 0.04 & 0.14 \\
\hline$[\mathrm{NdII} / \mathrm{H}]$ & 0.07 & 0.22 & 0.05 & 0.23 \\
\hline$[\mathrm{EuII} / \mathrm{H}]$ & 0.04 & 0.12 & 0.02 & 0.12 \\
\hline \multicolumn{5}{|c|}{ Error budget for HV $1328, \phi=0.884(\mathrm{MJD}=54785.02744873)$} \\
\hline Element & $\begin{array}{c}\Delta T_{\text {eff }} \\
( \pm 150 \mathrm{~K}) \\
(\mathrm{dex}) \\
\end{array}$ & $\begin{array}{c}\Delta \log g \\
( \pm 0.3 \mathrm{dex}) \\
(\mathrm{dex})\end{array}$ & $\begin{array}{c}\Delta V_{t} \\
\left( \pm 0.5 \mathrm{~km} \mathrm{~s}^{-1}\right) \\
(\mathrm{dex})\end{array}$ & $\begin{array}{c}\text { Quadratic } \\
\text { sum } \\
(\text { dex })\end{array}$ \\
\hline$[\mathrm{NaI} / \mathrm{H}]$ & 0.10 & 0.04 & 0.02 & 0.11 \\
\hline$[\mathrm{MgI} / \mathrm{H}]$ & 0.11 & 0.05 & 0.02 & 0.12 \\
\hline$[\mathrm{SiI} / \mathrm{H}]$ & 0.18 & 0.07 & 0.02 & 0.19 \\
\hline$[\mathrm{CaI} / \mathrm{H}]$ & 0.13 & 0.06 & 0.04 & 0.15 \\
\hline$[\mathrm{ScII} / \mathrm{H}]$ & 0.28 & 0.10 & 0.06 & 0.30 \\
\hline$[\mathrm{TiI} / \mathrm{H}]$ & 0.09 & 0.11 & 0.03 & 0.15 \\
\hline$[\mathrm{TiII} / \mathrm{H}]$ & 0.12 & 0.03 & 0.07 & 0.14 \\
\hline$[\mathrm{CrI} / \mathrm{H}]$ & 0.13 & 0.05 & 0.07 & 0.15 \\
\hline$[\mathrm{CrII} / \mathrm{H}]$ & 0.10 & 0.07 & 0.06 & 0.13 \\
\hline$[\mathrm{FeI} / \mathrm{H}]$ & 0.11 & 0.06 & 0.08 & 0.15 \\
\hline$[\mathrm{FeII} / \mathrm{H}]$ & 0.22 & 0.13 & 0.09 & 0.26 \\
\hline$[\mathrm{NiI} / \mathrm{H}]$ & 0.09 & 0.13 & 0.05 & 0.16 \\
\hline [YII/H] & 0.07 & 0.08 & 0.03 & 0.11 \\
\hline$[\mathrm{ZrII} / \mathrm{H}]$ & 0.31 & 0.12 & 0.02 & 0.33 \\
\hline$[\mathrm{LaII} / \mathrm{H}]$ & 0.13 & 0.05 & 0.03 & 0.14 \\
\hline$[\mathrm{NdII} / \mathrm{H}]$ & 0.10 & 0.15 & 0.03 & 0.18 \\
\hline$[\mathrm{EuII} / \mathrm{H}]$ & 0.28 & 0.08 & 0.03 & 0.29 \\
\hline
\end{tabular}

Notes. Columns 2, 3, and 4 indicate how the abundances are modified (mean values) when they are computed with over- or underestimated values of $T_{\text {eff }}( \pm 150 \mathrm{~K}), \log g( \pm 0.3 \mathrm{dex})$, or $V_{t}( \pm 0.5 \mathrm{dex})$, respectively. The sum in quadrature of the differences is adopted as the uncertainty in the abundances due to the uncertainties in the atmosphere parameters.

to the uncertainties in the atmosphere parameters. The resulting values are listed in Table 4.

For the stars in our sample, NLTE effects are negligible for $\mathrm{Na}(\leq 0.1 \mathrm{dex})$ as computed by Lind et al. (2011) for a range of atmospheric parameters including yellow supergiants and by Takeda et al. (2013) for Cepheids. Using DAOSPEC to automatically determine the EW of the lines (and the relatively low $\mathrm{S} / \mathrm{N}$ of our spectra) made it impossible for us to take into account the contribution of the hyperfine structure splitting for iron-peak elements, and for neutron-capture elements as in da Silva et al. (2016). Depending on the line considered, the latter authors estimated the hfs correction to range from negligible to $\approx 0.20$ dex. In the case of the $6262.29 \mathrm{La}$ II line, it reaches $-0.211 \pm 0.178 \mathrm{dex}$, where the quoted error represents the dispersion around the mean hfs correction for this line. In a forthcoming paper (Lemasle et al., in prep.) we study the impact of the hfs on the Mn abundance in Milky Way Cepheids. The three Mn lines measured in our Magellanic Cepheids belong to the $(6013,6016,6021 \AA)$ triplet and as expected we find lower Mn abundances when the hfs is taken into account. For the $6013 \AA$ lines, we find a mean effect of $-0.18 \pm 0.21$ dex (max: -0.65 dex), while it is slightly lower for the 6016 and $6021 \AA$ lines, with a mean effect of $-0.12 \pm 0.22$ dex (max: $-0.45 \mathrm{dex})$ and $-0.12 \pm 0.16$ dex (max: $-0.40 \mathrm{dex}$ ), respectively. It should be noted that the Milky Way Cepheids are on average more metalrich and somewhat cooler than the Magellanic Cepheids in our sample.

\section{Discussion}

\subsection{The chemical composition of Cepheids in NGC 1866}

The most striking feature of the abundance pattern of the NGC 1866 Cepheids is the very low star-to-star scatter (see Fig. 2): all the elements for which a good number of lines could be measured (e.g., $\mathrm{Si}, \mathrm{Ca}, \mathrm{Fe}$ ) have abundances $[\mathrm{X} / \mathrm{H}]$ that fall within $\approx 0.1$ dex of each other. The same also applies for other elements (e.g., S, Sc, Ti, Ni, Y, Zr) where only a small number of lines could be measured, and even in the case of, for example, $\mathrm{Na}$ or $\mathrm{Mg}$, where only one line could be measured, the scatter remains smaller than 0.2 dex. In a few cases (mostly for neutroncapture elements), a star has a discrepant abundance for a given element, either because this element could be measured (probably poorly) in only one of the spectra (e.g., Mn in HV 12199, La in HV 12203) or because one of the spectra gives a discrepant value (e.g., Nd for HV 12202 or Eu for HV 12197). Ignoring the outliers, the star to star scatter is similar to the one observed for the other elements.

This small star-to-star scatter is a strong indication that the six Cepheids in our NGC 1866 sample are genuine cluster members, sharing a very similar chemical composition as expected if they were born in the same place and at the same time. Indeed, they all have $2.64 \mathrm{~d}<P<3.52 \mathrm{~d}$ and it is well-known that classical Cepheids obey a period-age relation (e.g., Efremov 1978; Grebel \& Brandner 1998; Bono et al. 2005, see also Sect. 4.3).

With $[\mathrm{Fe} / \mathrm{H}] \approx-0.4 \mathrm{dex}$, our NGC 1866 Cepheids can be compared to Cepheids located in the outer disc of the Milky Way, at Galactocentric distances $R_{\mathrm{G}}>10 \mathrm{kpc}$. A quick glance at the Cepheid abundances in, for example, Lemasle et al. (2013), and Genovali et al. (2015) indicates that the $[\mathrm{Na} / \mathrm{Fe}]$ and $[\alpha / \mathrm{Fe}]$ abundances in the NGC 1866 Cepheids fall slightly below those observed in the Milky Way Cepheids for the corresponding range of metallicities. The same comparison for neutron-capture elements (in da Silva et al. 2016) is less meaningful as the low $\mathrm{S} / \mathrm{N}$ of our spectra prevented us from taking the hyperfine structure into account in the current study. The $[\mathrm{Y} / \mathrm{Fe}]$ ratios appear to be similar, which is not surprising as the hfs corrections reported by da Silva et al. (2016) are small for the YII lines. The [La/Fe], $[\mathrm{Nd} / \mathrm{Fe}]$, and $[\mathrm{Eu} / \mathrm{Fe}]$ ratios appear to be higher than in the Milky Way Cepheids with similar metallicities. This is certainly partially due to the hfs corrections. Indeed da Silva et al. (2016) report that the abundances derived from some of the La II lines can be smaller by up to $\approx 0.2 \mathrm{dex}$. On the other hand, their hfs corrections for the Eu lines are very small, and they did not apply any correction for $\mathrm{Nd}$, which indicates that at least a fraction of the difference is intrinsic. 


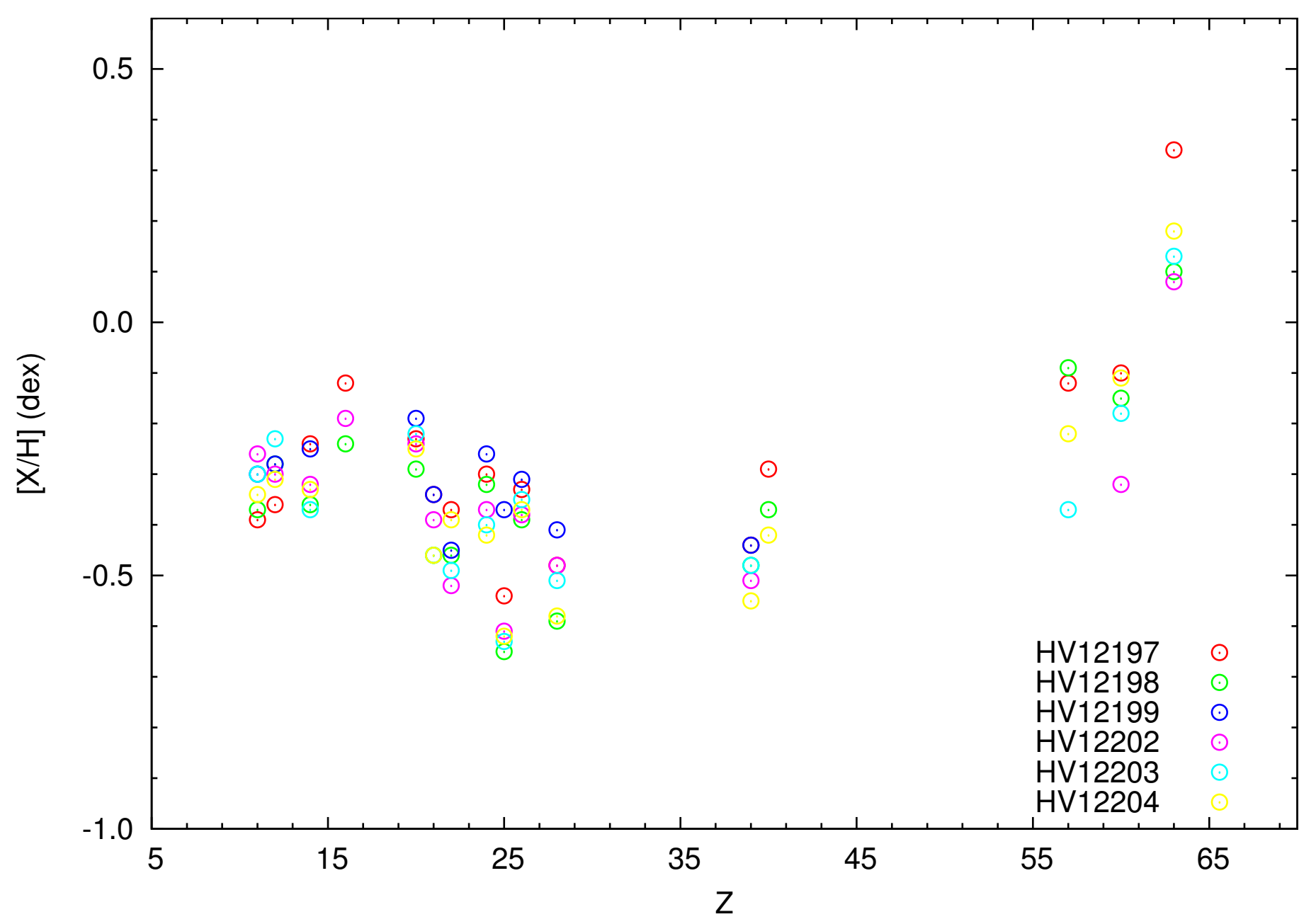

Fig. 2. Abundance ratios $([\mathrm{X} / \mathrm{H}])$ for our NGC 1866 Cepheids for different elements identified by their atomic number $Z$.

Cepheids embedded in open clusters are extremely important: as the clusters' distances can be determined independently via main sequence or isochrone fitting, their Cepheids can be used to calibrate the period-luminosity relations (e.g., Turner 2010). Furthermore, they can be used to establish periodage relations since the ages of star clusters can be determined from their resolved color-magnitude diagrams. The search for Cepheids as members of open clusters or OB associations was conducted in a long-term effort ${ }^{4}$ by, for example, Turner et al. (2012), Majaess et al. (2013), and references therein as well as by Anderson et al. (2013) and Chen et al. (2015) in recent extensive studies. They combined spatial (position, distance) and kinematic data with additional information (age, $[\mathrm{Fe} / \mathrm{H}]$ ) about the stellar populations of the open clusters and found roughly 30 Cepheids associated with open clusters in the Milky Way. However the maximum number of Cepheids that belong to a given cluster is two, much lower than the 23 Cepheids found in NGC 1866 (e.g., Welch \& Stetson 1993).

Comparing the detailed chemical composition of the Cepheids with the one of the other cluster members, as done for the first time in this paper (see Sect. 4.2), speaks in favor of the Cepheid membership of the cluster and should be considered in the future as an important criterion when seeking to match Cepheids to open clusters. This argument holds only if the photospheric abundances in this evolutionary phase were not

\footnotetext{
4 http://www.ap.smu.ca/ turner/cdlist.html
}

altered by stellar evolution. In the case of Cepheids, this is expected only for $\mathrm{C}, \mathrm{N}$ (the first dredge-up alters the surface composition of $\mathrm{C}$ and $\mathrm{N}$, and leaves $\mathrm{O}$ unaltered), and probably $\mathrm{Na}$ (the $\mathrm{Ne}-\mathrm{Na}$ cycle brings $\mathrm{Na}$-enriched material to the surface). As far as the Milky Way is concerned, the chemical composition of (RGB) stars in open clusters containing Cepheids is often missing, while the direct measurement of stellar abundances in more distant galaxies is out of reach for the current facilities, with the exception of bright red supergiants (RSGs, e.g., Davies et al. 2015; Patrick et al. 2015; Gazak et al. 2015). Obtaining detailed abundances from RSGs or cluster integrated-light spectroscopy (Colucci et al. 2012) for those extragalactic clusters harboring Cepheids would allow us to investigate the longstanding issue of a possible metallicity dependence of the period-luminosity relations that might affect the extragalactic distance scale (e.g., Romaniello et al. 2008).

\subsection{Comparison with giant stars in NGC 1866 and integrated-light spectroscopy}

Figure 3 shows a comparison of the abundance ratios $[\mathrm{X} / \mathrm{Fe}]$ between the six NGC 1866 Cepheids in our sample and other NGC 1866 stars: the 14 RGB stars of Mucciarelli et al. (2011) for which we show the mean abundance ratios and dispersions and the three stars of Colucci et al. (2012) displayed individually. We also overplot the cluster mean abundance derived from 
B. Lemasle et al.: Detailed chemical composition of classical Cepheids in the Magellanic Clouds

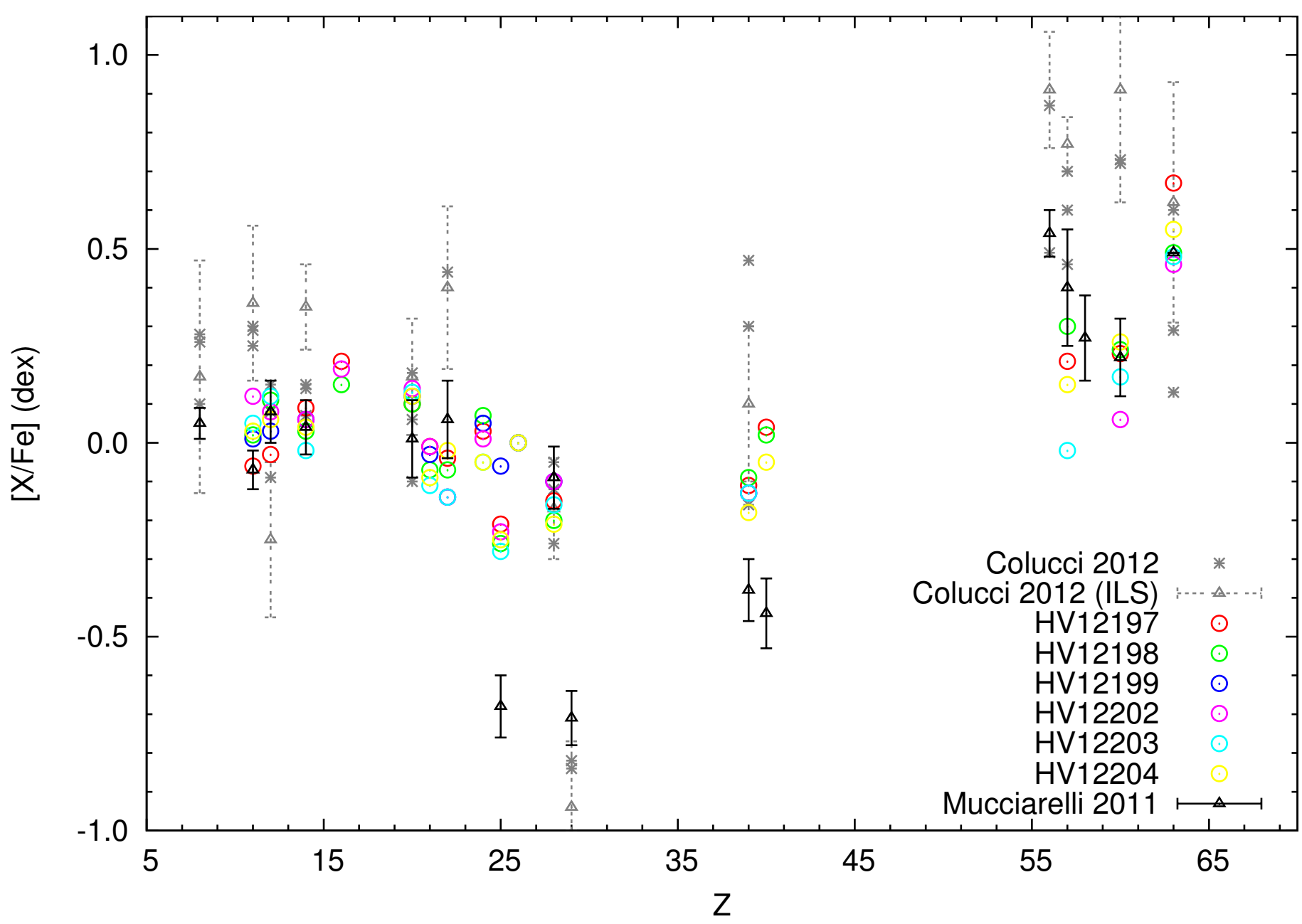

Fig. 3. Abundance ratios $([\mathrm{X} / \mathrm{Fe}])$ in NGC 1866 for different elements identified by their atomic number $Z$. Our Cepheids are the colored open circles, The mean value and dispersion for RGB stars in NGC 1866 from Mucciarelli et al. (2011) are given by the black triangle and solid line. Individual stellar abundances in NGC 1866 by Colucci \& Bernstein (2012) are depicted by gray stars. The mean value and dispersion obtained by Colucci \& Bernstein (2012) via integrated-light spectroscopy are indicated by the gray triangle and dotted line. All the abundance ratios have been rescaled to our solar reference values.

integrated-light spectroscopy by Colucci et al. (2012). All the abundances have been rescaled to our solar reference values.

Our Cepheids are slightly enriched in sodium with respect to the RGB stars of Mucciarelli et al. (2011). Similar Na overabundances have already been reported in the Milky Way (e.g., Genovali et al. 2015) when comparing Cepheids and field dwarfs in the thin and thick disc (Soubiran \& Girard 2005). Although this overabundance is probably partially due to NLTE effects (see Sect. 3.6), it has been proposed that it may be caused by mixing events that dredge up material enriched in $\mathrm{Na}$ via the $\mathrm{NeNa}$ cycle into the surface of the Cepheids (Sasselov 1986; Denissenkov 1994; Takeda et al. 2013). Similar Na overabundances have also been observed in RGB stars (e.g., da Silva et al. 2015), reinforcing this hypothesis. It is interesting to note that $\mathrm{Na}$ overabundances are relatively homogeneous in Cepheids and do not depend on mass or period (Andrievsky et al. 2003; Kovtyukh et al. 2005; Takeda et al. 2013; Genovali et al. 2015). In contrast, da Silva et al. (2015) report a positive trend with mass for $[\mathrm{Na} / \mathrm{Fe}]$ for RGB stars (which cover a shorter mass range).

The agreement is excellent for the $\alpha$-elements $\mathrm{Mg}$ and $\mathrm{Si}$, and to a lesser extent for $\mathrm{Ca}$ for which the Cepheid abundances are slightly larger than in the RGB stars. The agreement is good for $\mathrm{Fe}$ and excellent for $\mathrm{Ni}$, the only two iron peak elements for which data are available for both RGB stars and Cepheids. For our six Cepheids we find a mean $[\mathrm{Fe} / \mathrm{H}]=-0.36$ dex with a dispersion of 0.03 dex. The 14 RGB stars in Mucciarelli et al. (2011) have an average $[\mathrm{Fe} / \mathrm{H}]$ of $-0.43 \mathrm{dex}$ (to which one should add 0.02 dex to take into account differences in the adopted solar iron abundance) and a dispersion of 0.04 dex.

In contrast, the abundances of some neutron-capture elements are quite discrepant between the two studies: $\mathrm{Y}$ and $\mathrm{Zr}$ are found significantly more abundant (by $0.25 / 0.40$ dex respectively) than in Mucciarelli et al. (2011). Our abundances of La agree only within the error bars whereas $\mathrm{Nd}$ and Eu abundances are in excellent agreement with those reported by these authors. The hfs corrections reported by da Silva et al. (2016) are negligible for $\mathrm{Y}$ and therefore cannot account for the difference. In contrast, hfs corrections can reach -0.2 dex for several La lines, and a good agreement between both studies could be achieved if they were taken into account. A possible explanation for these discrepancies could be that the transitions used to derive the abundances of these elements are associated with different ionization stages. For instance, Allen \& Barbuy (2006, their Fig. 13) derived lower $\mathrm{Zr}$ abundances from $\mathrm{Zr}$ I lines than from $\mathrm{Zr}$ II lines in the Barium stars they analyzed, possibly because ionized lines 
are the dominant species and therefore less affected by departures from the LTE. In the end, their $\mathrm{Zr}$ II abundances span a range of $0.40 \leq[\mathrm{Zr} \mathrm{II} / \mathrm{Fe}] \leq 1.60$ while their $\mathrm{Zr}$ I abundances are found in the $-0.20 \leq[\mathrm{Zr} \mathrm{I} / \mathrm{Fe}] \leq 1.45$ range. Mucciarelli et al. (2011) do not provide their linelist but given the wavelength range of their spectra, it is likely that they used neutral lines. Unfortunately, neutral lines for these elements are too weak and/or blended in the spectra of Cepheids and therefore cannot be measured to test this hypothesis. Only the $\mathrm{Zr}$ I lines at 6134.58 and $6143.25 \AA$, and the Y I line at $6435.05 \AA$ could possibly be measured in the most metal-rich Milky Way Cepheids, but they become too weak already at solar metallicity.

The abundance ratios derived by Mucciarelli et al. (2011) for NGC 1866 members are in very good agreement with the field RGB stars in the surroundings they analyzed. It is interesting to note that the $[\mathrm{La} / \mathrm{Fe}]$ and $[\mathrm{Eu} / \mathrm{Fe}]$ ratios derived in NGC 1866 by Mucciarelli et al. (2011) are in good agreement with other LMC field RGB stars (e.g., Van der Swaelmen et al. 2013, and references therein), while their $[\mathrm{Y} / \mathrm{Fe}]$ and $[\mathrm{Zr} / \mathrm{Fe}]$ ratios fall at the lower end of the LMC field stars distribution.

$\mathrm{Y}$ and $\mathrm{Zr}$ belong to the first peak of the $s$-process, while La and $\mathrm{Ce}$ belong to the second peak of the $s$-process that is favored when metal-poor AGB stars dominate the chemical enrichment (e.g., Cristallo et al. 2011). The large values of [La/Fe] and $[\mathrm{Ce} / \mathrm{Fe}]$ demonstrate that the enrichment in heavy elements is dominated by metal-poor AGB stars for both the Cepheids and RGB stars in NGC 1866. Cepheids show higher $\mathrm{Y}$ and $\mathrm{Zr}$ abundances than RGB stars. If this difference turns out to be real, it might hint that they experienced extra enrichment in light $s$-process elements from more metal-rich AGB stars.

Similar conclusions can be drawn when comparing the Cepheid abundances with the stellar abundances derived by Colucci \& Bernstein (2012): the $\alpha$-elements (except Ti) and the iron-peak elements abundance ratios (with respect to iron) they obtained are very similar to those of the Cepheids, while their abundance ratios for the $n$-capture elements are higher than in the Cepheids, and even higher than those derived by Mucciarelli et al. (2011). Colucci \& Bernstein (2012) did not measure Mn in their NGC 1866 stellar sample. However, they found values $([\mathrm{Mn} / \mathrm{Fe}] \approx-0.35 \mathrm{dex})$ slightly lower than ours $([\mathrm{Mn} / \mathrm{Fe}] \approx-0.25 \mathrm{dex})$ in the stars belonging to other young LMC clusters. The Mn abundances reported by Mucciarelli et al. (2011) are also (much) lower than ours. This is almost certainly due to the fact that both studies included hfs corrections for Mn, which are known to be very significant (e.g., Prochaska et al. 2000). Because these ratios are lower than in Milky Way stars of the same metallicity, they proposed that the type Ia supernovae yields of Mn are metallicity-dependent, as reported/modeled in other environments by, for example, McWilliam et al. (2003), Cescutti et al. (2008), and North et al. (2012).

In contrast, the abundance ratios they derived from integrated-light spectroscopy are almost always significantly larger than those obtained for RGB stars by Mucciarelli et al (2011) or for Cepheids (this study), or at least at the higher end. This might be due to the fact that the work by Colucci et al. based on integrated-light includes contributions of many different stellar types (and possibly contaminating field populations). This is nevertheless surprising because the integrated flux originating from a young cluster such as NGC 1866 should be dominated by young supergiants, and one would therefore expect a better match between the Cepheids and the integrated-light spectroscopy abundance ratios.

\subsection{Multiple stellar populations in NGC 1866}

In a recent paper, Milone et al. (2017) reported the discovery of a split main sequence (MS) and of an extended main sequence turn-off in NGC 1866. These intriguing features have already been reported in many of the intermediate-age clusters in the Magellanic Clouds as well as for some of their young clusters (e.g., Bertelli et al. 2003; Glatt et al. 2008; Milone et al. 2013), although there is no agreement whether this is indeed due to multiple stellar populations. The blue MS hosts roughly $1 / 3$ of the MS stars, the remaining $2 / 3$ belonging to a spatially more concentrated red MS. Milone et al. (2017) rule out the possibility that age variations can be solely responsible for the split of the MS in NGC 1866. Instead, the red MS is consistent with a $\approx 200 \mathrm{Myr}$ old population of extremely fast-rotating stars $\left(\omega=0.9 \omega_{c}\right)$ while the blue MS is consistent with non-rotating stars of similar age, including a small fraction of even older stars. However, according to Milone et al. (2017) the upper blue MS can only be reproduced by a somewhat younger population $(\approx 140$ Myr old $)$ accounting for roughly $15 \%$ of the total MS stars.

As the age range of Cepheids is similar to the one of the NGC 1866 MS stars, it is natural to examine how they fit in the global picture of NGC 1866 drawn by Milone et al. (2017). These authors clearly state in their conclusion that the above interpretation should only be considered as a working hypothesis and our only intent here is to examine if Cepheids can shed some light on this scenario.

It is possible to compute individual ages for Cepheids with a period-age relation derived from pulsation models (e.g., Bono et al. 2005). Because rotation brings fresh material to the core during the MS hydrogen burning phase, fast-rotating stars of intermediate masses stay longer on the MS and therefore cross the instability strip later than a non-rotating star. Including rotation in models then increases the ages of Cepheids by 50 to $100 \%$, depending on the period, as computed by Anderson et al. (2016). Following the prescriptions of Anderson et al. (2016) we derive ages for all the Cepheids known in NGC 1866: we use a period-age relation computed with models with average rotation $\left(\omega=0.5 \omega_{c}\right)$ and averaged over the second and third crossing of the instability strip. Periods are taken from Musella et al. (2016). In the absence of further information, we assume that they are fundamental pulsators, except for V5, V6, and V8, as Musella et al. (2016) report that their periods and light curves are typical of first overtone pulsators. Even more importantly, they lie on the PL relations of first overtones. For comparison, we also derive ages using the period-age relation from Bono et al. (2005), which was computed using non-rotating pulsation models. Ages are listed in Table 5.

We first notice that in both cases the age spread is very limited, thus reinforcing previous findings stating that there is no age variation within NGC 1866, or at least that Cepheids all belong to the same sub-population. As expected, the ages calculated with the period-age relation from Bono et al. (2005) lead to younger Cepheids and therefore appear to be compatible only with the 140 Myr old stars populating the upper part of the blue main sequence. None of the period-age relations by Bono et al. (2005) and Anderson et al. (2016) enable us to compute individual error bars. Uncertainties on the ages of the NGC 1866 Cepheids of the order of 25-30 Myr can be derived by using the standard deviation of the periodage relation by Bono et al. (2005) as the error. However, given quoted error bars of $50 \%$ or more (Anderson et al. 2016), an age of 200 Myr cannot be completely excluded. On the other hand, the ages computed with the period-age 
Table 5. Individual ages for Cepheids in NGC 1866 computed with the period-age relations of Bono et al. (2005) or Anderson et al. (2016) for fundamental pulsators, and the periods listed in Musella et al. (2016).

\begin{tabular}{rccc}
\hline \hline Cepheid & Period & $\begin{array}{c}\text { Age }^{a} \\
\text { (no rotation) } \\
\text { (Myr) }\end{array}$ & $\begin{array}{c}\text { Age } \\
\left.\text { (rotation: } \omega=0.5 \omega_{c}\right) \\
(\mathrm{Myr})\end{array}$ \\
\hline V6 $^{c}$ & 1.9442620 & 114.5 & 258.7 \\
V8 $^{c}$ & 2.0070000 & 111.7 & 252.0 \\
V5 $^{c}$ & 2.0390710 & 110.3 & 248.7 \\
HV 12199 & 2.6391600 & 120.6 & 222.7 \\
HV 12200 & 2.7249800 & 117.6 & 218.0 \\
We 4 & 2.8603600 & 113.2 & 211.1 \\
WS 5 & 2.8978000 & 112.1 & 209.3 \\
New & 2.9429300 & 110.8 & 207.1 \\
HV 12203 & 2.9541100 & 110.4 & 206.6 \\
We 8 & 3.0398490 & 108.0 & 202.7 \\
We 3 & 3.0490400 & 107.7 & 202.3 \\
WS 11 & 3.0533000 & 107.6 & 202.1 \\
We 2 & 3.0548500 & 107.6 & 202.1 \\
WS 9 & 3.0694500 & 107.2 & 201.4 \\
V1 & 3.0845500 & 106.8 & 200.8 \\
HV 12202 & 3.1012000 & 106.3 & 200.0 \\
HV 12197 & 3.1437100 & 105.2 & 198.2 \\
We 5 & 3.1745000 & 104.4 & 197.0 \\
We 7 & 3.2322700 & 102.9 & 194.6 \\
We 6 & 3.2899400 & 101.5 & 192.3 \\
V4 & 3.3180000 & 100.9 & 191.3 \\
HV 12204 & 3.4388200 & 98.1 & 186.8 \\
V7 & 3.4520700 & 97.8 & 186.3 \\
HV 12198 & 3.5228000 & 96.3 & 183.8 \\
\hline & & & \\
\hline
\end{tabular}

Notes. ${ }^{(a)}$ Period-age relation from Bono et al. (2005). ${ }^{(b)}$ Period-age relation from Anderson et al. (2016). ${ }^{(c)}$ Ages computed using periodage relations for first overtone pulsators.

relation including rotation from Anderson et al. (2016) correspond very well to the fast-rotating red MS population. However the reader should keep in mind that ages should be directly compared only when they are on the same scale, which requires that they were all calculated based on the same models.

Using evolutionary tracks computed with either canonical (no overshooting) or non-canonical (moderate overshooting) assumptions (but no rotation), Musella et al. (2016) favor an age of 140 Myr. The location of the Cepheids, in between the theoretical blue loops computed in each case, does not allow us to discriminate between the two overshooting hypotheses. Adopting a canonical overshooting and an older age of 180 Myr enables us to better fit the observed luminosities of the Cepheids, but the theoretical blue loops are then too short to reach the Cepheids' locus in the CMD. Finally, using high-resolution integrated-light spectroscopy and CMD-fitting techniques, Colucci et al. (2011) report a similar age of $130 \mathrm{Myr}$.

Ages of Cepheids, derived using period-age relations computed with either no rotation or an average rotation $(\omega=$ $\left.0.5 \omega_{c}\right)$, do not allow us to confirm or rule out the hypothesis of Milone et al. (2017). Unfortunately, Anderson et al. (2016) do not provide period-age relations for fast-rotators $\left(\omega=0.9 \omega_{c}\right)$. As far as Cepheid ages are concerned, it is interesting to note that the Cepheids in NGC 1866 match very well the peak of the

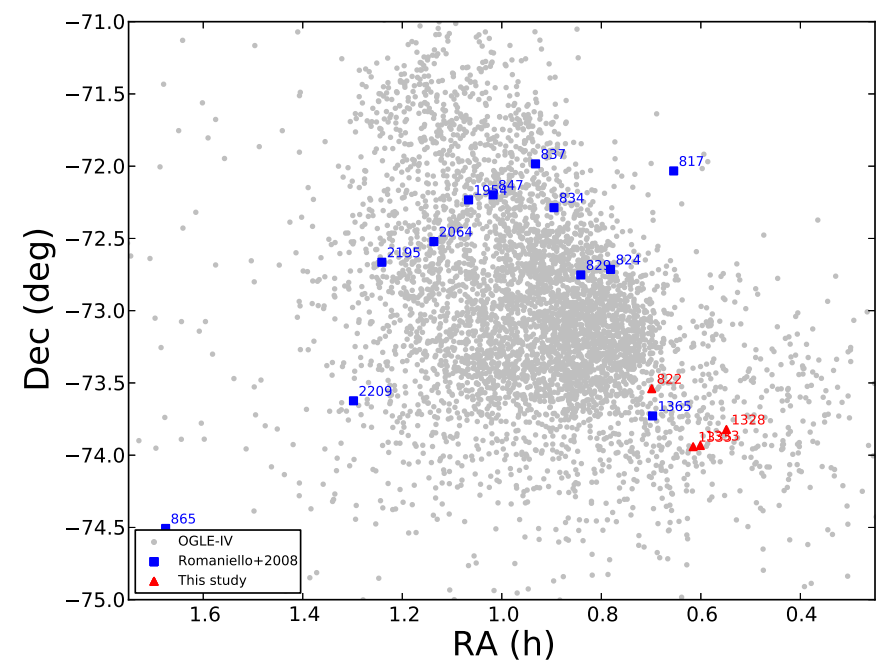

Fig. 4. SMC Cepheids with known metallicities (red: this study; blue: Romaniello et al. (2008); SMC Cepheids in the OGLE-IV database are shown as gray dots).

age distribution for LMC field Cepheids, computed by Inno et al. (2015b) using new period-age relations (without rotation) at LMC metallicities.

\subsection{The metallicity gradients from Cepheids in the SMC}

The existence of a metallicity gradient across the SMC is a longdebated issue. Using large numbers of RGB stars, Carrera et al. (2008), Dobbie et al. (2014), and Parisi et al. (2016) report a radial metallicity gradient $\left(-0.075 \pm 0.011 \mathrm{dex} \mathrm{deg}^{-1}\right.$ vs. $-0.08 \pm$ $0.02 \mathrm{dex} \mathrm{deg}^{-1}$ in the two latter studies) in the inner few degrees of the SMC. In both cases, this effect is attributed to the increasing fraction of younger, more metal-rich stars towards the SMC center. However, the presence of such a gradient was not confirmed by C- and M-type AGB stars (Cioni et al. 2009), populous clusters (e.g., Parisi et al. 2015, 2016, and references therein), or RR Lyrae studies (e.g., Haschke et al. 2012a; Deb et al. 2015; Skowron et al. 2016, and references therein).

The SMC is very elongated and tilted by more than $20^{\circ}$ (e.g., Haschke et al. 2012b; Subramanian et al. 2012; Nidever et al. 2013). Moreover, old and young stellar populations have significantly different spatial distributions and orientations (e.g., Haschke et al. 2012b; Jacyszyn-Dobrzeniecka et al. 2017). Recent studies using mid-infrared Spitzer data (Scowcroft et al. 2016) or optical data from the OGLE IV experiment (Jacyszyn-Dobrzeniecka et al. 2016) clearly confirmed this complex shape. Our Cepheid abundances combined with those found in the literature (Romaniello et al. 2008), and the possibility to derive accurate distances thanks to the period-luminosity relations allow us to shed new light on the SMC metallicity distribution. For the first time, we are able to probe the metallicity gradient in the SMC young population in the "depth" direction. As Cepheids are young stars, it should be noted that our study only concerns the present-day abundance gradient, and as such, the metal-rich end of the metallicity distribution function $([\mathrm{Fe} / \mathrm{H}]>-0.90 \mathrm{dex})$. Moreover, our sample is small (17 stars) and does not contain stars in the inner few degrees of the SMC in an on-sky projection (see Fig. 4). For old populations traced by RR Lyrae stars, no significant metallicity gradient was found in the "depth" direction (Haschke et al. 2012a). 
Table 6. Individual distances, ages, and metallicities for SMC Cepheids.

\begin{tabular}{rcccccc}
\hline \hline Cepheid & $\begin{array}{c}\log P \\
(\mathrm{~d})\end{array}$ & $\begin{array}{c}\text { Age }^{a} \\
(\mathrm{Myr})\end{array}$ & $\begin{array}{c}\text { Distance } \\
(\mathrm{MIR})(\mathrm{pc})\end{array}$ & $\begin{array}{c}\text { Distance } \\
(\mathrm{NIR})(\mathrm{pc})\end{array}$ & $\begin{array}{c}\text { Uncertainty on } \\
\text { NIR distances }(\mathrm{pc})\end{array}$ & $\begin{array}{c}{[\mathrm{Fe} / \mathrm{H}]} \\
(\mathrm{dex})\end{array}$ \\
\hline HV 817 & 1.277 & 212.4 & 57502 & $55636^{c}$ & 1136 & -0.79 \\
HV 823 & 1.504 & 186.9 & - & $60770^{b}$ & 964 & -0.77 \\
HV 824 & 1.819 & 161.2 & 56700 & $51195^{c}$ & 957 & -0.70 \\
HV 829 & 1.926 & 154.2 & 55506 & $53625^{c}$ & 964 & -0.73 \\
HV 834 & 1.867 & 157.9 & 57420 & $60463^{c}$ & 1168 & -0.60 \\
HV 837 & 1.631 & 175.5 & 60752 & $57692^{c}$ & 1033 & -0.80 \\
HV 847 & 1.433 & 194.2 & 63160 & $60129^{b}$ & 1175 & -0.72 \\
HV 865 & 1.523 & 185.2 & 54586 & $58401^{c}$ & 1018 & -0.84 \\
HV 1365 & 1.094 & 239.7 & 71595 & $68986^{b}$ & 1094 & -0.79 \\
HV 1954 & 1.222 & 219.8 & 54265 & $57702^{b}$ & 1027 & -0.73 \\
HV 2064 & 1.527 & 184.7 & 65503 & $58973^{c}$ & 1182 & -0.61 \\
HV 2195 & 1.621 & 176.3 & 58568 & $58703^{c}$ & 1163 & -0.64 \\
HV 2209 & 1.355 & 202.8 & 56286 & $56543^{b}$ & 1006 & -0.62 \\
HV 11211 & 1.330 & 205.8 & 54140 & $53884^{c}$ & 966 & -0.80 \\
\hline HV 822 & 1.224 & 219.6 & 67447 & $64428^{b}$ & 1022 & -0.70 \\
HV 1328 & 1.200 & 223.0 & 61750 & $61526^{b}$ & 976 & -0.63 \\
HV 1333 & 1.212 & 221.2 & 69244 & $69002^{b}$ & 1094 & -0.86 \\
HV 1335 & 1.158 & 229.3 & 69493 & $68231^{b}$ & 1214 & -0.78 \\
\hline
\end{tabular}

Notes. Metallicities from Romaniello et al. (2008) have been put on the same metallicity scale (by adding 0.03 dex to them) as our data. ${ }^{(a)}$ Periodage relation from Bono et al. (2005). ${ }^{(b)}$ Distance based on IRSF/SIRIUS near-infrared photometry (Kato et al. 2007). ${ }^{(c)}$ Distance based on 2MASS near-infrared photometry (Skrutskie et al. 2006).

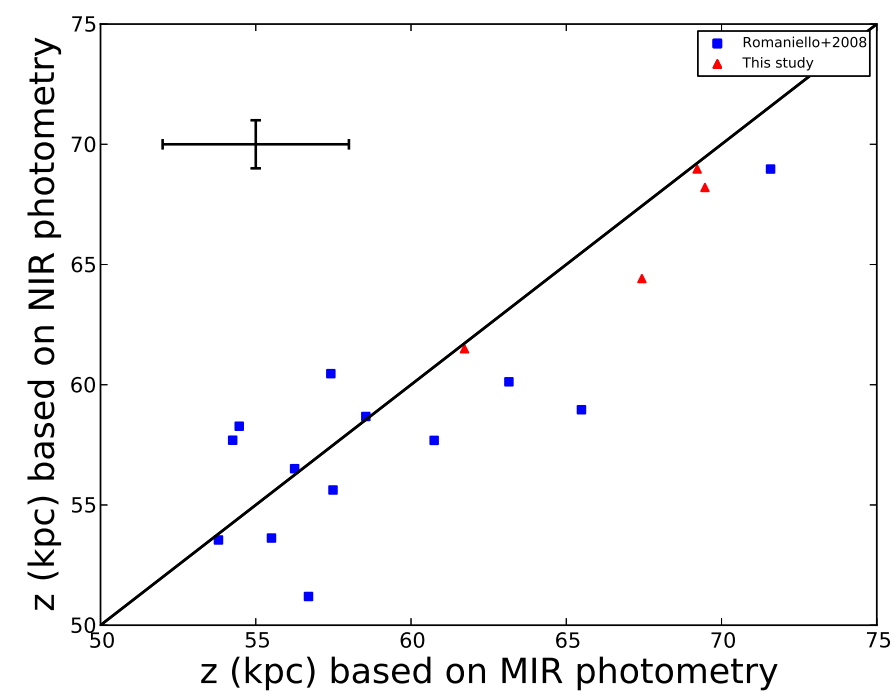

Fig. 5. Comparison of distances derived either from near-infrared or from mid-infrared photometry (red: this study; blue: Romaniello et al. 2008). Typical uncertainties are shown in the top left corner.

Individual distance moduli for SMC Cepheids were computed using the [3.6] $\mu \mathrm{m}$ mean-light magnitudes tabulated by Scowcroft et al. (2016) and the corresponding PL-relation in the mid-infrared (MIR) established by the same authors. Combining the extinction law of Indebetouw et al. (2005) with that of Cardelli et al. (1989), Monson et al. (2012) reported a total-toselective extinction ratio of $A_{[3.6]} / E(B-V)=0.203$. We adopted the average color excess found by Scowcroft et al. (2016) for the SMC: $E(B-V)=0.071 \pm 0.004 \mathrm{mag}$, which leads to $A_{[3.6]}=0.014 \pm 0.001 \mathrm{mag}$. There is no MIR photometry available for HV 822 and HV 823. For HV 822, we use the distance of 67441.4 pc derived by Groenewegen et al. (2013) via the Baade-Wesselink method. The typical uncertainty on the individual MIR distances is of the order of $\pm 3 \mathrm{kpc}$ (Scowcroft et al. 2016).

For comparison purposes, we also computed distances based on NIR photometry. For the Cepheids in the OGLE-IV database, we used near-infrared $J, H$ and $K_{\mathrm{S}}$ magnitudes from the IRSF/SIRIUS catalog (Kato et al. 2007) that were derived by using the near-infrared light-curve templates of Inno et al. (2015a). Distances were computed using period-Wesenheit (PW) relations calibrated on the entire SMC sample of fundamental mode Cepheids (>2200; stars Inno et al., in prep.). Wesenheit indices are reddening-free quantities by construction (Madore 1982). We used the $W_{H J K}$ index as defined by Inno et al. (2016): $W_{H J K}=$ $H-1.046 \times\left(J-K_{\mathrm{S}}\right)$ which is minimally affected by the uncertainty in the reddening law (Inno et al. 2016). For stars that are not in the OGLE-IV database, the same procedure was adopted, except that the distances are derived from 2MASS (Skrutskie et al. 2006) single epoch data (with no template applied). Individual uncertainties on distances are listed in Table 6. The typical uncertainty, computed as the average of the individual uncertainties, is $993 \pm 41 \mathrm{pc}$ and can be rounded to $1 \mathrm{kpc}$. It is beyond the scope of this paper to compare both sets of distances. We simply mention here that they are in very good agreement despite some star-to-star scatter (see Fig. 5).

To investigate the metallicity gradient in the SMC, we combine our $[\mathrm{Fe} / \mathrm{H}]$ abundances with those of Romaniello et al. (2008), to which we added 0.03 dex to take into account differences in the solar reference values. The Cepheids were placed in a Cartesian coordinate system using the transformations of van der Marel \& Cioni (2001) and Weinberg \& Nikolaev (2001). We adopted the value tabulated in SIMBAD for the center of the SMC: $\alpha_{0}=00 \mathrm{~h} 52 \mathrm{~m} 38.0 \mathrm{~s}, \delta_{0}=-72 \mathrm{~d} 48 \mathrm{~m} 01.00 \mathrm{~s}$ (J2000). For the SMC distance modulus, we adopted the value reported by Graczyk et al. (2014) using eclipsing binaries: $18.965 \pm 0.025$ (stat.) \pm 0.048 (syst.) mag which translates into a 


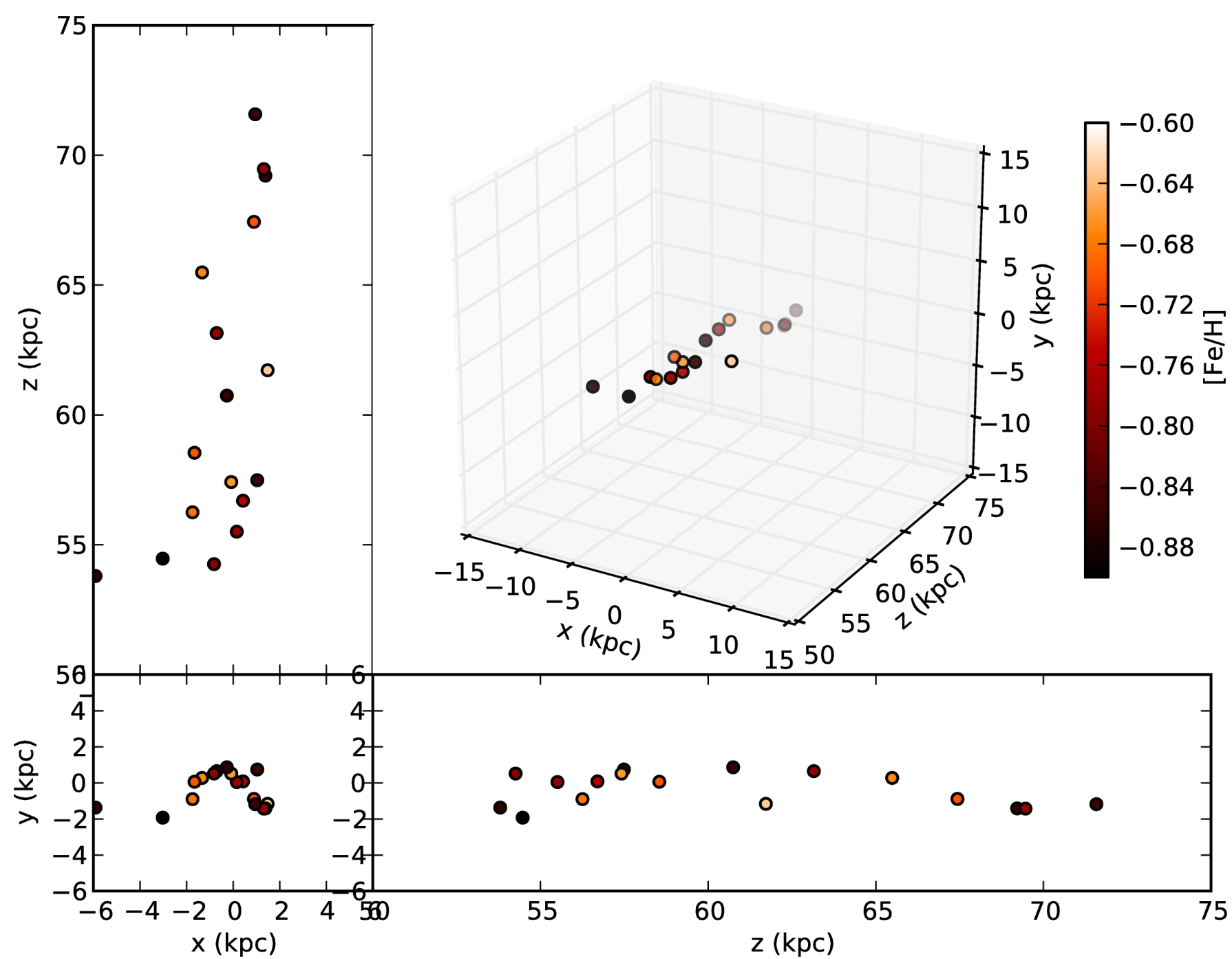

Fig. 6. Metallicity distribution of SMC Cepheids in Cartesian coordinates. Distances are based on mid-infrared photometry.

distance of $62.1 \pm 1.9 \mathrm{kpc}$. Individual distances and abundances can be found in Table 6, as well as ages derived with the periodage relation of Bono et al. (2005).

A first glance at Fig. 6 shows that the $(x, y)$ plane is not very relevant because it does not reflect the depth of the SMC. This fact is reinforced in the case of Cepheids as they are bright stars that can be easily identified and analyzed, even at very large distances. More interesting are the $(x, z)$ and especially the $(y, z)$ plane, as they allow us to study, for the first time, the metallicity distribution of Cepheids along the SMC main component. Our 17 Cepheids adequately sample the $z$ direction, but the reader should keep in mind that most of our targets are located above the main body of the SMC (see Fig. 4 or Jacyszyn-Dobrzeniecka et al. 2016, their Fig. 16). Figures 6 and 7 , where $[\mathrm{Fe} / \mathrm{H}]$ is plotted as a function of $z$, show no evidence of a metallicity gradient along the main axis of the SMC. The metallicity spread barely reaches $0.3 \mathrm{dex}$, but both ends of the $z$-axis seem to be slightly more metal poor than the inner regions as they miss the more metal-rich Cepheids. The age range spans only $100 \mathrm{Myr}$ and we see no correlation between age and metallicity or distance. These interesting findings should nevertheless only be considered as preliminary results, given the small size of our sample and the location of our Cepheids outside the main body of the SMC.

\section{Conclusions}

In this paper we conduct a spectroscopic analysis of Cepheids in the LMC and in the SMC. We provide abundances for a good number of $\alpha$, iron-peak, and neutron-capture elements. Our sample increases by $20 \%$ (respectively $25 \%$ ) the number of Cepheids with known metallicities and by $46 \%$ (respectively $50 \%$ ) the number of Cepheids with detailed chemical composition in these galaxies.

For the first time, we study the chemical composition of several Cepheids located in the same populous cluster NGC 1866, in the LMC. We find that the six Cepheids in our sample have a very homogeneous chemical composition, which is also consistent with RGB stars already analyzed in this cluster. Our results are also in good agreement with theoretical models accounting for luminosity and radial velocity variations for the two stars (HV 12197, HV 12199) for which such measurements are available. Using various versions of period-age relations with no $(\omega=0)$ or average rotation $\left(\omega=0.5 \omega_{c}\right)$ we find a similar age for 

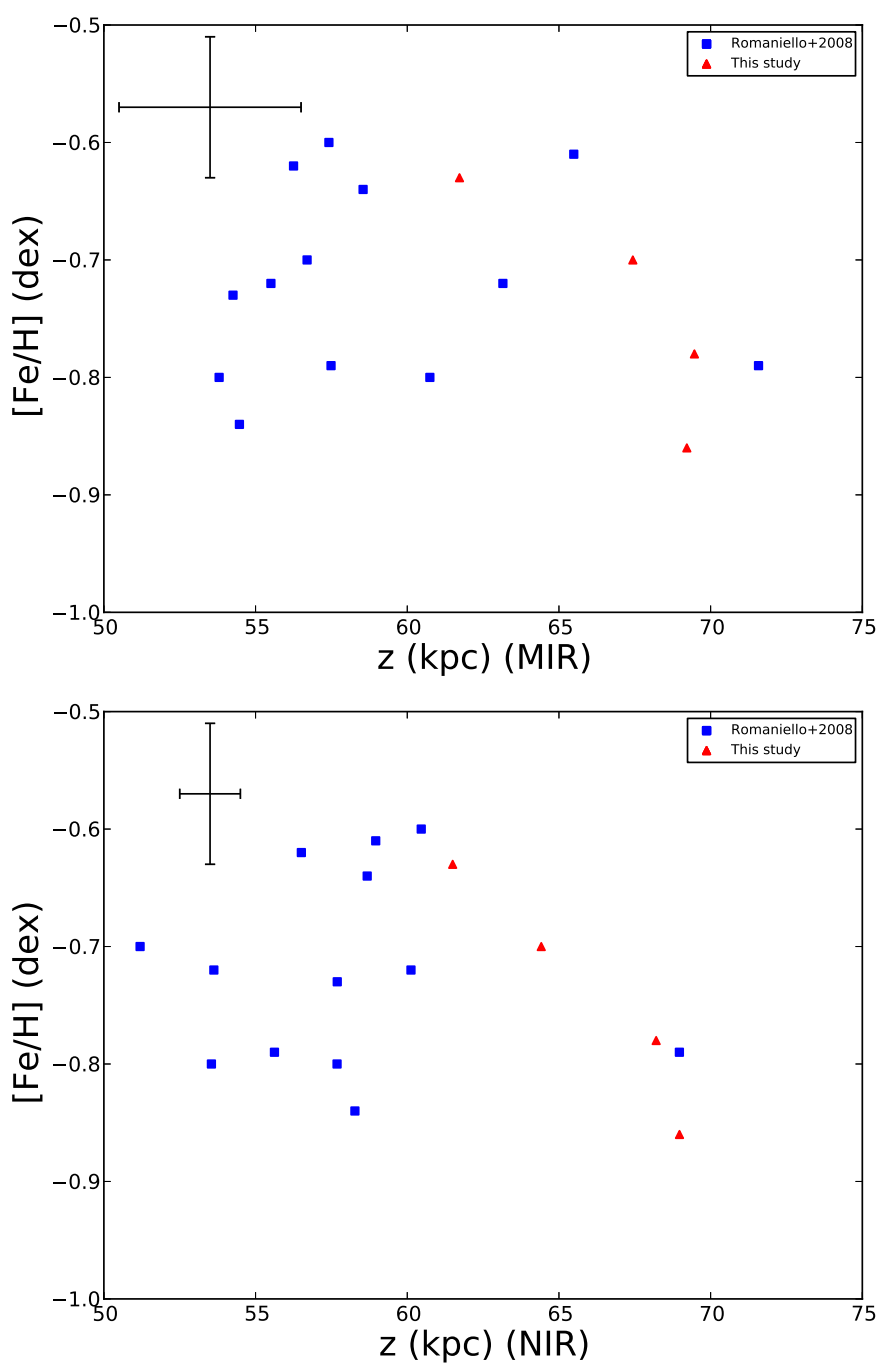

Fig. 7. SMC metallicity distribution from Cepheids in the $z$ (depth) direction. Top panel: distances derived from mid-infrared photometry; Bottom panel: distances derived from near-infrared photometry. Typical error bars are shown in the top left corner.

all the Cepheids in NGC 1866, indicating that they all belong to the same stellar population.

Using near- or mid-infrared photometry and periodluminosity relations (Inno et al. 2016; Scowcroft et al. 2016), we compute the distances for Cepheids in the SMC. Combining our abundances for Cepheids in the SMC with those of Romaniello et al. (2008), we study for the first time the metallicity distribution of the young population in the SMC in the depth direction. We find no metallicity gradient in the SMC, but our data include only a small number of stars and do not contain Cepheids in the inner few degrees of the SMC.

Acknowledgements. The authors would like to thank the referee, M. Van der Swaelmen, for his careful reading of the manuscript and for his valuable comments, which helped to improve the quality of this paper. This work was supported by Sonderforschungsbereich SFB 881 The Milky Way System (subproject A5) of the German Research Foundation (DFG). G.F. has been supported by the Futuro in Ricerca 2013 (grant RBFR13J716). This work has made use of the VALD database, operated at Uppsala University, the Institute of Astronomy RAS in Moscow, and the University of Vienna.

\section{References}

Allen, D. M., \& Barbuy, B. 2006, A\&A, 454, 895

Anders, E., \& Grevesse, N. 1989, Geochim. Cosmochim. Acta, 53, 1976 Anderson, R. I. 2014, A\&A, 566, L10

Anderson, R. I., Eyer, L., \& Mowlavi, N. 2013, MNRAS, 434, 2238

Anderson, R. I., Saio, H., Ekström, S., Georgy, C., \& Meynet, G. 2016, A\&A, 591, A8

Andrievsky, S. M., Kovtyukh, V. V., Luck, R. E., et al. 2002a, A\&A, 381, 32

Andrievsky, S. M., Bersier, D., Kovtyukh, V. V., et al. 2002b, A\&A, 384, 140

Andrievsky, S. M., Egorova, I. A., Korotin, S. A., \& Kovtyukh, V. V. 2003, Astron. Nachr., 324, 532

Andrievsky, S. M., Luck, R. E., \& Kovtyukh, V. V. 2005, AJ, 130, 1880

Andrievsky, S. M., Luck, R. E., \& Korotin, S. A. 2014, MNRAS, 437, 2106

Andrievsky, S. M., Martin, R. P., Kovtyukh, V. V., Korotin, S. A., \& Lépine, J. R. D. 2016, MNRAS, 461, 4256

Barnes, T. G., \& Evans, D. S. 1976, MNRAS, 174, 489

Benedict, G. F., McArthur, B. E., Feast, M. W., et al. 2007, AJ, 133, 1810

Bertelli, G., Nasi, E., Girardi, L., et al. 2003, AJ, 125, 770

Bhardwaj, A., Kanbur, S. M., Macri, L. M., et al. 2016, AJ, 151, 88

Bono, G., \& Marconi, M. 1997, MNRAS, 290, 353

Bono, G., Marconi, M., Cassisi, S., et al. 2005, ApJ, 621, 966

Bono, G., Caputo, F., Marconi, M., \& Musella, I. 2010, ApJ, 715, 277

Breitfelder, J., Mérand, A., Kervella, P., et al. 2016, A\&A, 587, A117

Brocato, E., Caputo, F., Castellani, V., Marconi, M., \& Musella, I. 2004, AJ, 128, 1597

Cardelli, J. A., Clayton, G. C., \& Mathis, J. S. 1989, ApJ, 345, 245

Carrera, R., Gallart, C., Aparicio, A., et al. 2008, AJ, 136, 1039,

Cescutti, G., Matteucci, F., Lanfranchi, G. A., \& McWilliam, A. 2008, A\&A, 491, 401

Chen, X., de Grijs, R., \& Deng, L. 2015, MNRAS, 446, 1268

Cioni, M.-R. L. 2009, A\&A, 506, 1137

Colucci, J. E., \& Bernstein, R. A. 2012, ApJ, 749, 124

Colucci, J. E., Bernstein, R. A., Cameron, S. A., \& McWilliam, A. 2011, ApJ, 735,55

Colucci, J. E., Bernstein, R. A., Cameron, S. A., \& McWilliam, A. 2012, ApJ, 746, 29

Cristallo, S., Piersanti, L., Straniero, O., et al. 2011, ApJS, 197, 17

da Silva, R., Milone, A. C., \& Rocha-Pinto, H. J. 2015, A\&A, 580, A24

da Silva, R., Lemasle, B., Bono, G., et al. 2016, A\&A, 586, A125

Davies, B., Kudritzki, R.-P., Gazak, Z., et al. 2015, ApJ, 806, 21

Deb, S., Singh, H. P., Kumar, S., \& Kanbur, S. M. 2015, MNRAS, 449, 2768

Dekker, H., D’ Odorico, S., Kaufer, A., Delabre, B., \& Kotzlowski, H. 2000, Proc. SPIE, 4008, 534

Denissenkov, P. A. 1994, A\&A, 287, 113

Dobbie, P. D., Cole, A. A., Subramaniam, A., \& Keller, S. 2014, MNRAS, 442, 1680

Efremov, Iu. N. 1978, Soviet. Astron., 22, 161

Feast, M. W., Whitelock, P. A., Menzies, J. W., \& Matsunaga, N. 2012, MNRAS, 421, 2998

Fiorentino, G., Marconi, M., Musella, I., \& Caputo, F. 2007, A\&A, 476, 863

Fouqué, P., \& Gieren, W. P. 1997, A\&A, 320, 799

Fouqué, P., Arriagada, P., Storm, J., et al. 2007, A\&A, 476, 73

Freudling, W., Romaniello, M., Bramich, D. M., et al. 2013, A\&A, 559, A96

Gazak, J. Z., Kudritzki, R., Evans, C., et al. 2015, ApJ, 805, 182

Genovali, K., Lemasle, B., Bono, G., et al. 2013, A\&A, 554, A132

Genovali, K., Lemasle, B., Bono, G., et al. 2014, A\&A, 566, A37

Genovali, K., Lemasle, B., da Silva, R., et al. 2015, A\&A, 580, A17

Gieren, W., Storm, J., Barnes, T., G., III, et al. 2005, ApJ, 627, 224

Gieren, W., Górski, M., Pietrzyński, G., et al. 2013, ApJ, 773, 69

Glatt, K., Grebel, E. K., Sabbi, E., et al. 2008, AJ, 136, 1703

Graczyk, D., Pietrzy/'nski, G., Thompson, I. B., et al. 2014, ApJ, 780, 59

Grebel, E. K., \& Brandner, W. 1998, in Magellanic Clouds and Other Dwarf

Galaxies, eds. T. Richtler, \& J. M. Braun (Aachen: Shaker Verlag), 151

Groenewegen, M. A. T. 2008, A\&A, 488, 25

Groenewegen, M. A. T. 2013, A\&A, 550, 70

Gustafsson, B., Edvardsson, B., Eriksson, K., et al. 2008, A\&A, 486, 951

Haschke, R., Grebel, E. K., \& Duffau, S. 2012a, AJ, 143, 48

Haschke, R., Grebel, E. K., \& Duffau, S. 2012b, AJ, 144, 107

Heiter, U., Lind, K., Asplund, M., et al. 2015, Phys. Scr., 90, 054010

Hill, V., Andrievsky, S. M., \& Spite, M. 1995, A\&A, 293, 347

Hill, V., François, P., Spite, M., Primas, F., \& Spite, F. 2000, A\&A, 364, 19

Indebetouw, R., Mathis, J. S., Babler, B. L., et al. 2005, ApJ, 619, 931

Inno, L., Matsunaga, N., Bono, G., et al. 2013, ApJ, 764, 84

Inno, L., Matsunaga, N., Romaniello, M., et al. 2015a, A\&A, 576, A30

Inno, L., Bono, G., Romaniello, M., et al. 2015b, ASP Conf., 491, 265

Inno, L., Bono, G., Matsunaga, N., et al. 2016, ApJ, 832, 176 
Jacyszyn-Dobrzeniecka, A. M., Skowron, D. M., Mróz, P., et al. 2016, Acta Astron., 66, 149

Jacyszyn-Dobrzeniecka, A. M., Skowron, D. M., Mróz, P., et al. 2017, Acta Astron., 67, 1

Kato, D., Nagashima, C., Nagayama, T., et al. 2007, PASJ, 59, 6150

Kervella, P., Nardetto, N., Bersier, D., Mourard, D., \& Coudé du Foresto, V. 2004, A\&A, 416, 941

Kervella, P., Trahin, B., Bond, H. E., et al. 2017, A\&A, 600, A127

Kewley, L. J., \& Ellison, S. L. 2008, ApJ, 681, 1183

Korotin, S. A., Andrievsky, S. M., Luck, R. E., et al. 2014, MNRAS, 444, 3301

Kovtyukh, V. V., \& Andrievsky, S. M. 1999, A\&A, 351, 597

Kovtyukh, V. V., \& Gorlova, N. I. 2000, A\&A, 358, 587

Kovtyukh, V. V., Wallerstein, G., \& Andrievsky, S. M. 2005, PASP, 117, 1173

Kupka, F., Piskunov, N. E., Ryabchikova, T. A., Stempels, H. C., \& Weiss, W. W. 1999, A\&AS, 138, 119

Lemasle, B., François, P., Bono, G., et al. 2007, A\&A, 467, 283

Lemasle, B., François, P., Piersimoni, A., et al. 2008, A\&A, 490, 613

Lemasle, B., François, P., Genovali, K., et al. 2013, A\&A, 558, A31

Lind, K., Asplund, M., Barklem, P. S., \& Belyaev, A. K. 2011, A\&A, 528, 103

Luck, R. E., \& Lambert, D. L. 1985, ApJ, 298, 782

Luck, R. E., \& Lambert, D. L. 1992, ApJS, 79, 303

Luck, R. E., \& Lambert, D. L. 2011, AJ, 142, 136

Luck, R. E., Moffett, T. J., Barnes, III, T. G., \& Gieren, W. P. 1998, AJ, 115, 605

Luck, R. E., Gieren, W. P., Andrievsky, S. M., et al. 2003, A\&A, 401, 939

Luck, R. E., Andrievsky, S. M., Kovtyukh, V. V., Gieren, W., \& Graczyk, D. 2011, AJ, 142, 51

Madore, B. F. 1982, ApJ, 253, 575

Majaess, D., Carraro, G., Moni Bidin, C., et al. 2013, A\&A, 560, A22

Marconi, M., Molinaro, R., Ripepi, V., Musella, I., \& Brocato, E. 2013, MNRAS, 428, 2185

Marconi, M., Molinaro, R., Ripepi, V., et al. 2017, MNRAS, 466, 3206

Martin, R. P., Andrievsky, S. M., Kovtyukh, V. V., et al. 2015, MNRAS, 449 4071

McWilliam, A., Rich, R. M., \& Smecker-Hane, T. A. 2003, ApJ, 592, L21

Mérand, A., Kervella, P., Breitfelder, J., et al. 2015, A\&A, 584, A80

Milone, A. P., Bedin, L. R., Cassisi, S., et al. 2013, A\&A, 555, A143

Milone, A. P., Marino, A. F., D'Antona, F., et al. 2017, MNRAS, 465, 4363

Molinaro, R., Ripepi, V., Marconi, M., et al. 2012, ApJ, 748, 69

Monson, A. J., Freedman, W. L., Madore, B. F., et al. 2012, ApJ, 759, 146

Mourard, D., Bonneau, D., Koechlin, L., et al. 1997, A\&A, 317, 789

Mucciarelli, A., Cristallo, S., Brocato, E., et al. 2011, MNRAS, 413, 837

Musella, I., Marconi, M., Stetson, P. B., et al. 2016, MNRAS, 457, 3084

Nardetto, N., Fokin, A., Fouqué, P., et al. 2011, A\&A, 534, L16

Nardetto, N., Poretti, E., Rainer, M., et al. 2014, A\&A, 561, A151

Neilson, H. R., Nardetto, N., Ngeow, C.-C., Fouqué, P., \& Storm, J. 2012, A\&A, 541, A 134

Ngeow, C.-C., Citro, D. M., \& Kanbur, S. M. 2012, MNRAS, 420, 585

Ngeow, C.-C., Sarkar, S., Bhardwaj, A., Kanbur, S. M., \& Singh, H. P. 2015, ApJ, 813, 57

Nidever, D. L., Monachesi, A., Bell, E. F., et al. 2013, ApJ, 779, 145
North, P., Cescutti, G., Jablonka, P., et al. 2012, A\&A, 541, A45 Parisi, M. C., Geisler, D., Clariá, J. J., et al. 2015, AJ, 149, 154 Parisi, M. C., Geisler, D., Carraro, G., et al. 2016, AJ, 152, 58 Pasquini, L., Avila, G., Blecha, A., et al. 2002, The Messenger, 110, 1 Patrick, L. R., Evans, C. J., Davies, B., et al. 2015, ApJ, 803, 14 Pedicelli, S., Lemasle, B., Groenewegen, M., et al. 2010, A\&A, 518, A11 Pilyugin, L. S., \& Grebel, E. K. 2016, MNRAS, 457, 3678

Prochaska, J. X., Naumov, S. O., Carney, B. W., McWilliam, A., \& Wolfe, A. M. 2000, AJ, 120, 2513

Rich, J. A., Persson, S. E., Freedman, W. L., et al. 2014, ApJ, 794, 107

Ripepi, V., Moretti, M. I., Marconi, M., et al. 2012, MNRAS, 424, 1807

Romaniello, M., Primas, F., Mottini, M, et al. 2005, A\&A, 429, 37

Romaniello, M., Primas, F., Mottini, M., et al. 2008, A\&A, 488, 731

Sasselov, D. D. 1986, PASP, 98, 561

Scowcroft, V., Freedman, W., L., Madore, B. F., et al. 2013, ApJ, 773, 106

Scowcroft, V., Freedman, W., L., Madore, B. F., et al. 2016, ApJ, 816, 49S

Skowron, D. M., Soszyński, I., Udalski, A., et al. 2016, Acta Astron., 66, 269

Skrutskie, M. F., Cutri, R. M., Stiening, R., et al. 2006, AJ, 131, 1163

Soszyński, I., Poleski, R., Udalski, A., et al. 2010, Acta Astron., 60, 17

Soubiran, C., \& Girard, P. 2005, A\&A, 438, 139

Spite, M. 1967, Ann. Astrophys., 30, 211

Spite, F., Spite, M., \& François, P. 1989, A\&A, 210, 25

Stetson, P. B., \& Pancino, E. 2008, PASP, 120, 1332

Storm, J., Carney, B. W., Gieren, W. P., et al. 2004a, A\&A, 415, 521

Storm, J., Carney, B. W., Gieren, W. P., et al. 2004b, A\&A, 415, 531

Storm, J., Gieren, W. P., Fouqué, P., Barnes, III, T. G., \& Gómez, M. 2005, A\&A 440,487

Storm, J., Gieren, W., Fouqué, P., et al. 2011a, A\&A, 534, A94

Storm, J., Gieren, W., Fouqué, P., et al. 2011b, A\&A, 534, A95

Subramanian, S., \& Subramaniam, A. 2012, ApJ, 744, 128

Szabados, L. 2003, IBVS, 5394

Szabados, L., \& Nehź, D. 2012, MNRAS, 426, 3148

Takeda, Y., Kang, D.-I., Han, I., Lee, B.-C., \& Kim, K.-M. 2013, MNRAS, 432, 769

Turner, D. G. 2010, Ap\&SS, 326, 219

Turner, D. G., Majaess, D. J., Lane, D. J., et al. 2012, MNRAS, 422, 2501

Udalski, A., Szymanski, M. K., \& Szymanski, G. 2015, Acta Astron., 65, 1

van der Marel, R. P., \& Cioni, M.-R. L. 2001, AJ, 122, 1807

Van der Swaelmen, M., Hill, V., Primas, F., \& Cole, A. A. 2013, A\&A, 560, A44

van Leeuwen, F., Feast, M. W., Whitelock, P. A., \& Laney, C. D. 2007, MNRAS, 379,723

Weinberg, M. D., \& Nikolaev, S. 2001, ApJ, 548, 712

Welch, D. L. 1994, AJ, 108, 1421

Welch, D. L., \& Stetson, P. B. 1993, AJ, 105, 1813

Welch, D. L., Mateo, M., Côté, P., Fischer, P., \& Madore, B. F. 1991, AJ, 101, 490

Wesselink, A. J. 1969, MNRAS, 144, 297

Yong, D., Carney, B. W., Teixera de Almeida, M. L., \& Pohl, B. L. 2006, AJ, 131,2256 


\section{Appendix A: Abundances of the Cepheids in the LMC cluster NGC 1866}

Table A.1. Chemical composition of HV 12197.

\begin{tabular}{|c|c|c|c|c|c|c|c|c|c|c|c|}
\hline HV 12197 & \multicolumn{3}{|c|}{$\mathrm{MJD}=54806.02457622$} & \multicolumn{3}{|c|}{$\mathrm{MJD}=54806.08071473$} & \multicolumn{3}{|c|}{$\mathrm{MJD}=54806.13684767$} & \multicolumn{2}{|c|}{ Abundance } \\
\hline Element & $\begin{array}{r}{[\mathrm{X} / \mathrm{H}]} \\
(\mathrm{dex})\end{array}$ & $\begin{array}{c}\sigma \\
(\operatorname{dex})\end{array}$ & $N$ & $\begin{array}{r}{[\mathrm{X} / \mathrm{H}]} \\
(\mathrm{dex})\end{array}$ & $\begin{array}{c}\sigma \\
(\operatorname{dex})\end{array}$ & $N$ & $\begin{array}{r}{[\mathrm{X} / \mathrm{H}]} \\
(\mathrm{dex})\end{array}$ & $\begin{array}{c}\sigma \\
(\operatorname{dex})\end{array}$ & $N$ & $\begin{array}{r}{[\mathrm{X} / \mathrm{H}]} \\
(\mathrm{dex})\end{array}$ & $\begin{array}{c}\sigma \\
(\operatorname{dex})\end{array}$ \\
\hline$[\mathrm{NaI} / \mathrm{H}]$ & -0.33 & & 1 & -0.37 & & 1 & -0.46 & & 1 & -0.39 & 0.04 \\
\hline$[\mathrm{MgI} / \mathrm{H}]$ & -0.36 & & 1 & -0.43 & & 1 & -0.29 & & 1 & -0.36 & 0.04 \\
\hline $\begin{array}{r}{[\mathrm{SiI} / \mathrm{H}]} \\
{[\mathrm{SiII} / \mathrm{H}]}\end{array}$ & -0.16 & 0.18 & 3 & -0.21 & 0.19 & 5 & -0.31 & 0.10 & 5 & -0.24 & 0.09 \\
\hline$[\mathrm{SI} / \mathrm{H}]$ & -0.06 & & 1 & -0.24 & & 1 & -0.06 & & 1 & -0.12 & 0.06 \\
\hline$[\mathrm{CaI} / \mathrm{H}]$ & -0.19 & 0.06 & 10 & -0.26 & 0.06 & 10 & -0.23 & 0.08 & 11 & -0.23 & 0.04 \\
\hline$[\mathrm{ScII} / \mathrm{H}]$ & -0.30 & 0.19 & 6 & -0.34 & 0.12 & 6 & -0.37 & 0.16 & 6 & -0.34 & 0.09 \\
\hline [Til/H] & -0.55 & & 1 & -0.27 & 0.35 & 2 & & & & -0.36 & 0.22 \\
\hline [TiII/H] & -0.52 & & 1 & -0.36 & 0.07 & 2 & -0.31 & 0.16 & 2 & -0.37 & 0.07 \\
\hline$[\mathrm{CrI} / \mathrm{H}]$ & -0.24 & & 1 & & & & & & & -0.24 & \\
\hline$[\mathrm{CrII} / \mathrm{H}]$ & -0.16 & 0.11 & 3 & -0.39 & 0.13 & 3 & -0.35 & 0.07 & 4 & -0.30 & 0.06 \\
\hline$[\mathrm{MnI} / \mathrm{H}]$ & & & & -0.55 & 0.04 & 2 & -0.52 & & 1 & -0.54 & 0.03 \\
\hline$[\mathrm{FeI} / \mathrm{H}]$ & -0.31 & 0.10 & 27 & -0.34 & 0.11 & 50 & -0.34 & 0.13 & 59 & -0.33 & 0.07 \\
\hline$[\mathrm{FeII} / \mathrm{H}]$ & -0.34 & 0.16 & 8 & -0.33 & 0.09 & 8 & -0.33 & 0.12 & 9 & -0.33 & 0.07 \\
\hline$[\mathrm{NiI} / \mathrm{H}]$ & -0.38 & & 1 & -0.49 & 0.17 & 3 & -0.49 & 0.17 & 5 & -0.48 & 0.10 \\
\hline [YII/H] & -0.45 & & 1 & -0.46 & 0.06 & 2 & -0.41 & 0.15 & 2 & -0.44 & 0.05 \\
\hline$[\mathrm{ZrII} / \mathrm{H}]$ & & & & -0.31 & & 1 & -0.27 & & 1 & -0.29 & 0.02 \\
\hline$[\mathrm{LaII} / \mathrm{H}]$ & & & & -0.17 & & 1 & -0.09 & 0.16 & 2 & -0.12 & 0.10 \\
\hline$[\mathrm{NdII} / \mathrm{H}]$ & & & & -0.09 & 0.08 & 3 & -0.12 & 0.06 & 2 & -0.10 & 0.05 \\
\hline [EuII/H] & 0.48 & & 1 & 0.29 & & 1 & 0.26 & & 1 & 0.34 & 0.07 \\
\hline
\end{tabular}

Notes. For a given element, the abundance (computed as the mean value of the abundances determined for each individual line of this element), $\mathrm{rms}$, and number of lines $(N)$ used is given for each individual spectrum analyzed. The final abundance of a star is computed as the weighted mean (and standard deviation) for the three spectra analyzed, where the weight is the number of lines of a given element measured in each spectrum. We remind the reader that hfs was not taken into account and that the hfs correction might range from negligible to severe (up to -0.2 dex; da Silva et al. 2016) for $\mathrm{La}$ and (up to $-0.65 \mathrm{dex}$, Lemasle et al., in prep.) for $\mathrm{Mn}$, while it is expected to be negligible for $\mathrm{Y}, \mathrm{Zr}$, $\mathrm{Nd}$, and Eu. 
B. Lemasle et al.: Detailed chemical composition of classical Cepheids in the Magellanic Clouds

Table A.2. Same as Table A.1 for HV 12198.

\begin{tabular}{|c|c|c|c|c|c|c|c|c|c|c|c|}
\hline HV 12198 & \multicolumn{3}{|c|}{$\mathrm{MJD}=54806.02457622$} & \multicolumn{3}{|c|}{$\mathrm{MJD}=54806.08071473$} & \multicolumn{3}{|c|}{$\mathrm{MJD}=54806.13684767$} & \multicolumn{2}{|c|}{ Abundance } \\
\hline Element & $\begin{array}{r}{[\mathrm{X} / \mathrm{H}]} \\
(\mathrm{dex})\end{array}$ & $\begin{array}{c}\sigma \\
(\mathrm{dex})\end{array}$ & $N$ & $\begin{array}{r}{[\mathrm{X} / \mathrm{H}]} \\
(\mathrm{dex})\end{array}$ & $\begin{array}{c}\sigma \\
(\mathrm{dex})\end{array}$ & $N$ & $\begin{array}{r}{[\mathrm{X} / \mathrm{H}]} \\
(\mathrm{dex})\end{array}$ & $\begin{array}{c}\sigma \\
(\operatorname{dex})\end{array}$ & $N$ & $\begin{array}{r}{[\mathrm{X} / \mathrm{H}]} \\
(\mathrm{dex})\end{array}$ & $\begin{array}{c}\sigma \\
(\mathrm{dex})\end{array}$ \\
\hline$[\mathrm{NaI} / \mathrm{H}]$ & -0.37 & 0.05 & 2 & -0.33 & & 1 & -0.40 & 0.13 & 2 & -0.37 & 0.05 \\
\hline$[\mathrm{MgI} / \mathrm{H}]$ & -0.34 & & 1 & -0.27 & & 1 & -0.23 & & 1 & -0.28 & 0.04 \\
\hline$[\mathrm{SiI} / \mathrm{H}]$ & -0.34 & 0.04 & 2 & -0.31 & 0.28 & 5 & -0.39 & 0.08 & 8 & -0.36 & 0.09 \\
\hline$[\mathrm{SiII} / \mathrm{H}]$ & & & & -0.21 & & 1 & -0.27 & & 1 & -0.24 & 0.03 \\
\hline$[\mathrm{SI} / \mathrm{H}]$ & & & & & & & -0.24 & & 1 & -0.24 & \\
\hline$[\mathrm{CaI} / \mathrm{H}]$ & -0.35 & 0.14 & 3 & -0.26 & 0.08 & 4 & -0.26 & 0.05 & 3 & -0.29 & 0.05 \\
\hline$[\mathrm{ScII} / \mathrm{H}]$ & -0.50 & 0.06 & 2 & -0.44 & 0.21 & 3 & -0.44 & 0.10 & 3 & -0.46 & 0.08 \\
\hline$[\mathrm{TiI} / \mathrm{H}]$ & -0.43 & & 1 & -0.48 & 0.08 & 3 & -0.46 & 0.15 & 3 & -0.46 & 0.07 \\
\hline [TiII/H] & -0.31 & & 1 & -0.34 & & 1 & -0.47 & & 1 & -0.37 & 0.05 \\
\hline$[\mathrm{CrI} / \mathrm{H}]$ & -0.48 & & 1 & & & & & & & -0.48 & \\
\hline$[\mathrm{CrII} / \mathrm{H}]$ & -0.23 & 0.17 & 2 & -0.35 & 0.16 & 3 & -0.34 & 0.09 & 3 & -0.32 & 0.07 \\
\hline$[\mathrm{MnI} / \mathrm{H}]$ & -0.64 & 0.05 & 2 & -0.71 & 0.04 & 2 & -0.57 & & 1 & -0.65 & 0.03 \\
\hline$[\mathrm{Fel} / \mathrm{H}]$ & -0.39 & 0.10 & 33 & -0.37 & 0.10 & 50 & -0.40 & 0.07 & 43 & -0.39 & 0.05 \\
\hline$[\mathrm{FeII} / \mathrm{H}]$ & -0.41 & 0.16 & 4 & -0.38 & 0.11 & 5 & -0.40 & 0.09 & 8 & -0.40 & 0.06 \\
\hline$[\mathrm{NiI} / \mathrm{H}]$ & -0.51 & 0.16 & 4 & -0.59 & 0.16 & 6 & -0.62 & 0.10 & 8 & -0.59 & 0.07 \\
\hline [YII/H] & -0.38 & 0.13 & 2 & -0.46 & 0.15 & 3 & -0.57 & 0.15 & 3 & -0.48 & 0.08 \\
\hline$[\mathrm{ZrII} / \mathrm{H}]$ & & & & & & & -0.37 & & 1 & -0.37 & \\
\hline [LaII/H] & -0.05 & 0.01 & 2 & -0.07 & 0.03 & 2 & -0.16 & 0.01 & 2 & -0.09 & 0.02 \\
\hline$[\mathrm{NdII} / \mathrm{H}]$ & -0.16 & 0.09 & 2 & -0.15 & 0.05 & 3 & -0.15 & 0.08 & 3 & -0.15 & 0.04 \\
\hline [EuII/H] & 0.10 & & 1 & 0.12 & & 1 & 0.09 & 0.16 & 2 & 0.10 & 0.07 \\
\hline
\end{tabular}

Table A.3. Same as Table A.1 for HV 12199.

\begin{tabular}{|c|c|c|c|c|c|c|c|c|c|c|c|}
\hline HV 12199 & MJD $=$ & 54806 & 622 & MJD $=$ & 54806 & 473 & MJD $=$ & 54806 & 767 & Abul & ance \\
\hline Element & $\begin{array}{r}{[\mathrm{X} / \mathrm{H}]} \\
(\mathrm{dex})\end{array}$ & $\begin{array}{c}\sigma \\
(\operatorname{dex})\end{array}$ & $N$ & $\begin{array}{r}{[\mathrm{X} / \mathrm{H}]} \\
(\mathrm{dex})\end{array}$ & $\begin{array}{c}\sigma \\
(\mathrm{dex})\end{array}$ & $N$ & $\begin{array}{r}{[\mathrm{X} / \mathrm{H}]} \\
(\mathrm{dex})\end{array}$ & $\begin{array}{c}\sigma \\
(\mathrm{dex})\end{array}$ & $N$ & $\begin{array}{r}{[\mathrm{X} / \mathrm{H}]} \\
(\mathrm{dex})\end{array}$ & $\begin{array}{c}\sigma \\
(\mathrm{dex})\end{array}$ \\
\hline$[\mathrm{NaI} / \mathrm{H}]$ & -0.33 & & 1 & -0.27 & 0.03 & 2 & -0.31 & 0.01 & 2 & -0.30 & 0.02 \\
\hline$[\mathrm{MgI} / \mathrm{H}]$ & -0.26 & & 1 & -0.31 & & 1 & -0.27 & & 1 & -0.28 & 0.01 \\
\hline $\begin{array}{r}{[\mathrm{SiI} / \mathrm{H}]} \\
{[\mathrm{SiII} / \mathrm{H}]}\end{array}$ & -0.23 & 0.11 & 2 & -0.31 & 0.05 & 3 & -0.20 & 0.09 & 3 & -0.25 & 0.04 \\
\hline $\begin{array}{r}{[\mathrm{SI} / \mathrm{H}]} \\
{[\mathrm{CaI} / \mathrm{H}]}\end{array}$ & -0.17 & 0.12 & 10 & -0.22 & 0.06 & 11 & -0.17 & 0.11 & 11 & -0.19 & 0.05 \\
\hline$[\mathrm{ScII} / \mathrm{H}]$ & -0.32 & 0.21 & 3 & -0.35 & 0.10 & 5 & -0.34 & 0.11 & 6 & -0.34 & 0.07 \\
\hline$[\mathrm{TiI} / \mathrm{H}]$ & & & & -0.47 & & 1 & & & & -0.47 & \\
\hline [TiII/H] & -0.44 & 0.05 & 3 & -0.48 & 0.03 & 2 & -0.40 & & 1 & -0.45 & 0.03 \\
\hline$[\mathrm{CrI} / \mathrm{H}]$ & -0.25 & & 1 & & & & -0.36 & & 1 & -0.30 & 0.05 \\
\hline$[\mathrm{CrII} / \mathrm{H}]$ & -0.29 & 0.26 & 3 & -0.16 & 0.08 & 2 & -0.29 & 0.18 & 4 & -0.26 & 0.11 \\
\hline$[\mathrm{MnI} / \mathrm{H}]$ & & & & & & & -0.37 & 0.18 & 2 & -0.37 & 0.18 \\
\hline$[\mathrm{Fel} / \mathrm{H}]$ & -0.29 & 0.10 & 29 & -0.30 & 0.05 & 35 & -0.32 & 0.10 & 49 & -0.31 & 0.05 \\
\hline$[\mathrm{FeII} / \mathrm{H}]$ & -0.30 & 0.09 & 8 & -0.30 & 0.11 & 9 & -0.31 & 0.15 & 11 & -0.30 & 0.07 \\
\hline$[\mathrm{Nil} / \mathrm{H}]$ & -0.33 & 0.15 & 3 & -0.42 & & 1 & -0.53 & 0.06 & 2 & -0.41 & 0.08 \\
\hline $\begin{array}{r}{[\mathrm{YII} / \mathrm{H}]} \\
{[\mathrm{ZrII} / \mathrm{H}]} \\
{[\mathrm{LaII} / \mathrm{H}]} \\
{[\mathrm{NdII} / \mathrm{H}]} \\
{[\mathrm{EuII} / \mathrm{H}]}\end{array}$ & -0.32 & & 1 & -0.48 & & 1 & -0.48 & 0.08 & 2 & -0.44 & 0.05 \\
\hline
\end{tabular}


Table A.4. Same as Table A.1 for HV 12202.

\begin{tabular}{|c|c|c|c|c|c|c|c|c|c|c|c|}
\hline HV 12202 & \multicolumn{3}{|c|}{$\mathrm{MJD}=54806.02457622$} & \multicolumn{3}{|c|}{$\mathrm{MJD}=54806.08071473$} & \multicolumn{3}{|c|}{$\mathrm{MJD}=54806.13684767$} & \multicolumn{2}{|c|}{ Abundance } \\
\hline Element & $\begin{array}{r}\mathrm{X} / \mathrm{H}] \\
(\mathrm{dex})\end{array}$ & $\begin{array}{c}\sigma \\
(\operatorname{dex})\end{array}$ & $N$ & $\begin{array}{r}{[\mathrm{X} / \mathrm{H}]} \\
(\mathrm{dex})\end{array}$ & $\begin{array}{c}\sigma \\
(\mathrm{dex})\end{array}$ & $N$ & $\begin{array}{r}{[\mathrm{X} / \mathrm{H}]} \\
(\mathrm{dex})\end{array}$ & $\begin{array}{c}\sigma \\
(\operatorname{dex})\end{array}$ & $N$ & $\begin{array}{r}{[\mathrm{X} / \mathrm{H}]} \\
(\mathrm{dex})\end{array}$ & $\begin{array}{c}\sigma \\
(\operatorname{dex})\end{array}$ \\
\hline$[\mathrm{NaI} / \mathrm{H}]$ & -0.31 & & 1 & -0.26 & & 1 & -0.23 & 0.06 & 2 & -0.26 & $\mathbf{0 . 0 3}$ \\
\hline$[\mathrm{MgI} / \mathrm{H}]$ & -0.28 & & 1 & -0.32 & & 1 & -0.30 & & 1 & $-\mathbf{0 . 3 0}$ & 0.01 \\
\hline$[\mathrm{SiI} / \mathrm{H}]$ & -0.31 & 0.18 & 3 & -0.31 & 0.14 & 4 & -0.33 & 0.03 & 3 & -0.32 & 0.07 \\
\hline$[\mathrm{SiII} / \mathrm{H}]$ & & & & -0.17 & & 1 & & & & -0.17 & \\
\hline$[\mathrm{SI} / \mathrm{H}]$ & & & & -0.09 & & 1 & -0.29 & & 1 & -0.19 & 0.10 \\
\hline$[\mathrm{CaI} / \mathrm{H}]$ & -0.35 & 0.13 & 9 & -0.21 & 0.10 & 9 & -0.17 & 0.08 & 9 & -0.24 & 0.06 \\
\hline$[\mathrm{ScII} / \mathrm{H}]$ & -0.45 & 0.23 & 3 & -0.39 & 0.20 & 3 & -0.30 & 0.03 & 2 & -0.39 & 0.10 \\
\hline [TiI/H] & -0.68 & & 1 & -0.48 & & 1 & -0.40 & & 1 & -0.52 & 0.08 \\
\hline [TiII/H] & & & & -0.40 & & 1 & & & & -0.40 & \\
\hline$[\mathrm{CrI} / \mathrm{H}]$ & & & & & & & & & & & \\
\hline [CrII/H] & -0.32 & 0.14 & 4 & -0.40 & 0.19 & 3 & -0.40 & 0.14 & 3 & $-\mathbf{0 . 3 7}$ & 0.08 \\
\hline$[\mathrm{MnI} / \mathrm{H}]$ & -0.71 & 0.02 & 1 & -0.58 & 0.03 & 3 & & & & -0.61 & 0.04 \\
\hline$[\mathrm{FeI} / \mathrm{H}]$ & -0.38 & 0.08 & 29 & -0.41 & 0.07 & 44 & -0.36 & 0.10 & 41 & -0.38 & 0.05 \\
\hline$[\mathrm{FeII} / \mathrm{H}]$ & -0.39 & 0.05 & 6 & -0.40 & 0.15 & 5 & -0.38 & 0.14 & 6 & -0.39 & 0.06 \\
\hline$[\mathrm{NiI} / \mathrm{H}]$ & -0.54 & 0.03 & 3 & -0.53 & 0.08 & 2 & -0.41 & 0.12 & 4 & -0.48 & 0.06 \\
\hline [YII/H] & -0.40 & 0.01 & & -0.54 & & 1 & -0.58 & & 1 & -0.51 & 0.05 \\
\hline [ZrII/H] & & & & & & & & & & & \\
\hline$[\mathrm{LaII} / \mathrm{H}]$ & & & & & & & & & & & \\
\hline$[\mathrm{NdII} / \mathrm{H}]$ & -0.25 & & 1 & & & & -0.39 & & 1 & -0.32 & 0.07 \\
\hline$[\mathrm{EuII} / \mathrm{H}]$ & & & & 0.08 & & 1 & & & & 0.08 & \\
\hline
\end{tabular}

Table A.5. Same as Table A.1 for HV 12203.

\begin{tabular}{|c|c|c|c|c|c|c|c|c|c|c|c|}
\hline \multirow{2}{*}{$\begin{array}{r}\text { HV } 12203 \\
\text { Element }\end{array}$} & \multicolumn{3}{|c|}{$\mathrm{MJD}=54806.02457622$} & \multicolumn{3}{|c|}{ MJD = 54806.08071473} & \multicolumn{3}{|c|}{ MJD = 54806.13684767} & \multicolumn{2}{|c|}{ Abundance } \\
\hline & $\begin{array}{r}{[\mathrm{X} / \mathrm{H}]} \\
(\mathrm{dex})\end{array}$ & $\begin{array}{c}\sigma \\
(\operatorname{dex})\end{array}$ & $N$ & $\begin{array}{r}{[\mathrm{X} / \mathrm{H}]} \\
(\mathrm{dex}) \\
\end{array}$ & $\begin{array}{c}\sigma \\
(\operatorname{dex})\end{array}$ & $N$ & $\begin{array}{r}{[\mathrm{X} / \mathrm{H}]} \\
(\mathrm{dex}) \\
\end{array}$ & $\begin{array}{c}\sigma \\
(\operatorname{dex})\end{array}$ & $N$ & $\begin{array}{r}{[\mathrm{X} / \mathrm{H}]} \\
(\mathrm{dex}) \\
\end{array}$ & $\begin{array}{c}\sigma \\
(\mathrm{dex})\end{array}$ \\
\hline$[\mathrm{NaI} / \mathrm{H}]$ & & & & -0.30 & & 1 & & & & -0.30 & \\
\hline$[\mathrm{MgI} / \mathrm{H}]$ & -0.18 & & 1 & -0.18 & & 1 & -0.34 & & 1 & -0.23 & 0.05 \\
\hline$[\mathrm{SiI} / \mathrm{H}]$ & -0.28 & 0.12 & 3 & -0.38 & 0.05 & 3 & -0.41 & 0.10 & 5 & -0.37 & 0.05 \\
\hline $\begin{array}{r}{[\mathrm{SiII} / \mathrm{H}]} \\
{[\mathrm{SI} / \mathrm{H}]}\end{array}$ & & & & & & & -0.23 & & 1 & -0.23 & \\
\hline$[\mathrm{CaI} / \mathrm{H}]$ & -0.25 & 0.13 & 4 & -0.18 & 0.10 & 9 & -0.25 & 0.12 & 6 & -0.22 & 0.06 \\
\hline$[\mathrm{ScII} / \mathrm{H}]$ & -0.33 & 0.22 & 5 & -0.55 & 0.20 & 3 & -0.57 & 0.02 & 3 & -0.46 & 0.11 \\
\hline $\begin{array}{r}{[\mathrm{TiI} / \mathrm{H}]} \\
{[\mathrm{TiII} / \mathrm{H}]} \\
{[\mathrm{CrI} / \mathrm{H}]}\end{array}$ & & & & -0.46 & & 1 & -0.53 & & 1 & -0.49 & 0.04 \\
\hline$[\mathrm{CrII} / \mathrm{H}]$ & -0.25 & 0.33 & 2 & -0.46 & 0.13 & 4 & -0.42 & 0.22 & 3 & -0.40 & 0.11 \\
\hline$[\mathrm{MnI} / \mathrm{H}]$ & -0.71 & & 1 & -0.59 & 0.11 & 2 & -0.64 & 0.06 & 2 & -0.63 & 0.05 \\
\hline$[\mathrm{FeI} / \mathrm{H}]$ & -0.33 & 0.14 & 32 & -0.36 & 0.08 & 37 & -0.36 & 0.12 & 45 & -0.35 & 0.07 \\
\hline$[\mathrm{FeII} / \mathrm{H}]$ & -0.36 & 0.06 & 5 & -0.39 & 0.11 & 2 & -0.35 & 0.16 & 8 & -0.36 & 0.09 \\
\hline$[\mathrm{NiI} / \mathrm{H}]$ & -0.51 & 0.20 & 5 & -0.45 & 0.06 & 3 & -0.56 & 0.20 & 4 & -0.51 & 0.10 \\
\hline [YII/H] & -0.34 & 0.19 & 2 & -0.62 & 0.11 & 2 & -0.49 & 0.11 & 2 & -0.48 & 0.08 \\
\hline$[\mathrm{ZrII} / \mathrm{H}]$ & & & & & & & & & & & \\
\hline$[\mathrm{LaII} / \mathrm{H}]$ & & & & -0.37 & 0.01 & 2 & & & & -0.37 & 0.01 \\
\hline$[\mathrm{NdII} / \mathrm{H}]$ & & & & -0.24 & 0.16 & 2 & -0.12 & 0.13 & 2 & -0.18 & 0.09 \\
\hline$[\mathrm{EuII} / \mathrm{H}]$ & & & & 0.13 & 0.06 & 2 & & & & 0.13 & 0.06 \\
\hline
\end{tabular}


B. Lemasle et al.: Detailed chemical composition of classical Cepheids in the Magellanic Clouds

Table A.6. Same as Table A.1 for HV 12204.

\begin{tabular}{|c|c|c|c|c|c|c|c|c|c|c|c|}
\hline HV 12204 & \multicolumn{3}{|c|}{$\mathrm{MJD}=54806.02457622$} & \multicolumn{3}{|c|}{$\mathrm{MJD}=54806.08071473$} & \multicolumn{3}{|c|}{$\mathrm{MJD}=54806.13684767$} & \multicolumn{2}{|c|}{ Abundance } \\
\hline Element & $\begin{array}{r}{[\mathrm{X} / \mathrm{H}]} \\
(\mathrm{dex})\end{array}$ & $\begin{array}{c}\sigma \\
(\operatorname{dex})\end{array}$ & $N$ & $\begin{array}{r}{[\mathrm{X} / \mathrm{H}]} \\
(\mathrm{dex})\end{array}$ & $\begin{array}{c}\sigma \\
(\operatorname{dex})\end{array}$ & $N$ & $\begin{array}{r}{[\mathrm{X} / \mathrm{H}]} \\
(\mathrm{dex})\end{array}$ & $\begin{array}{c}\sigma \\
(\operatorname{dex})\end{array}$ & $N$ & $\begin{array}{r}{[\mathrm{X} / \mathrm{H}]} \\
(\mathrm{dex})\end{array}$ & $\begin{array}{c}\sigma \\
(\mathrm{dex})\end{array}$ \\
\hline$[\mathrm{NaI} / \mathrm{H}]$ & -0.37 & 0.08 & 2 & -0.32 & 0.05 & 2 & -0.34 & & 1 & -0.34 & 0.03 \\
\hline$[\mathrm{MgI} / \mathrm{H}]$ & -0.34 & & 1 & -0.26 & & 1 & -0.32 & & 1 & -0.31 & 0.02 \\
\hline$[\mathrm{SiI} / \mathrm{H}]$ & -0.34 & 0.08 & 6 & -0.36 & 0.02 & 6 & -0.30 & 0.12 & 6 & -0.33 & 0.05 \\
\hline $\begin{array}{r}{[\mathrm{SiII} / \mathrm{H}]} \\
{[\mathrm{SI} / \mathrm{H}]}\end{array}$ & -0.21 & & 1 & -0.18 & & 1 & -0.22 & & 1 & -0.20 & 0.01 \\
\hline$[\mathrm{CaI} / \mathrm{H}]$ & -0.28 & 0.05 & 9 & -0.21 & 0.07 & 10 & -0.26 & 0.05 & 6 & -0.25 & 0.03 \\
\hline [ScII/H] & -0.47 & 0.19 & 5 & -0.47 & 0.15 & 6 & -0.44 & 0.08 & 4 & -0.46 & 0.08 \\
\hline$[\mathrm{TiI} / \mathrm{H}]$ & -0.46 & 0.13 & 2 & -0.42 & 0.11 & 3 & -0.33 & 0.27 & 4 & -0.39 & 0.12 \\
\hline [TiII/H] & -0.64 & & 1 & -0.45 & 0.08 & 3 & -0.43 & 0.06 & 3 & -0.47 & 0.05 \\
\hline$[\mathrm{CrI} / \mathrm{H}]$ & & & & -0.56 & & 1 & -0.53 & & 1 & -0.55 & 0.02 \\
\hline$[\mathrm{CrII} / \mathrm{H}]$ & -0.47 & 0.06 & 2 & -0.38 & 0.13 & 4 & -0.46 & 0.28 & 2 & -0.42 & 0.08 \\
\hline$[\mathrm{MnI} / \mathrm{H}]$ & -0.64 & & 1 & -0.61 & & 1 & -0.60 & & 1 & -0.62 & 0.01 \\
\hline$[\mathrm{Fel} / \mathrm{H}]$ & -0.38 & 0.07 & 44 & -0.34 & 0.10 & 56 & -0.38 & 0.10 & 57 & -0.37 & 0.05 \\
\hline$[\mathrm{FeII} / \mathrm{H}]$ & -0.38 & 0.14 & 9 & -0.35 & 0.09 & 10 & -0.39 & 0.10 & 11 & -0.37 & 0.06 \\
\hline$[\mathrm{NiI} / \mathrm{H}]$ & -0.51 & 0.10 & 5 & -0.60 & 0.10 & 7 & -0.60 & 0.14 & 7 & -0.58 & 0.07 \\
\hline$[\mathrm{YII} / \mathrm{H}]$ & -0.54 & 0.02 & 3 & -0.43 & 0.11 & 2 & -0.62 & 0.09 & 4 & -0.55 & 0.05 \\
\hline$[\mathrm{ZrII} / \mathrm{H}]$ & & & & -0.42 & & 1 & -0.43 & & 1 & -0.42 & 0.01 \\
\hline [LaII/H] & -0.17 & & 1 & -0.25 & 0.18 & 3 & -0.21 & 0.15 & 5 & -0.22 & 0.10 \\
\hline$[\mathrm{NdII} / \mathrm{H}]$ & -0.06 & 0.26 & 2 & -0.11 & 0.04 & 3 & -0.15 & 0.02 & 3 & -0.11 & 0.06 \\
\hline [EuII/H] & & & & 0.18 & & 1 & & & & 0.18 & \\
\hline
\end{tabular}


Appendix B: Abundances of the Cepheids in the SMC field

Table B.1. Same as Table A.1 for HV 822.

\begin{tabular}{|c|c|c|c|c|c|c|c|c|c|c|c|}
\hline \multirow{2}{*}{$\begin{array}{l}\text { HV } 822 \\
\text { Element }\end{array}$} & \multicolumn{3}{|c|}{$\mathrm{MJD}=54785.04026326$} & \multicolumn{3}{|c|}{$\mathrm{MJD}=54785.05240828$} & \multicolumn{3}{|c|}{$\mathrm{MJD}=54785.06455378$} & \multicolumn{2}{|c|}{ Abundance } \\
\hline & $\begin{array}{r}{[\mathrm{X} / \mathrm{H}]} \\
(\mathrm{dex})\end{array}$ & $\begin{array}{c}\sigma \\
(\operatorname{dex})\end{array}$ & $N$ & $\begin{array}{r}{[\mathrm{X} / \mathrm{H}]} \\
(\mathrm{dex})\end{array}$ & $\begin{array}{c}\sigma \\
(\operatorname{dex})\end{array}$ & $N$ & $\begin{array}{r}{[\mathrm{X} / \mathrm{H}]} \\
(\mathrm{dex})\end{array}$ & $\begin{array}{c}\sigma \\
(\mathrm{dex})\end{array}$ & $N$ & $\begin{array}{r}{[\mathrm{X} / \mathrm{H}]} \\
(\mathrm{dex})\end{array}$ & $\begin{array}{c}\sigma \\
(\mathrm{dex})\end{array}$ \\
\hline$[\mathrm{NaI} / \mathrm{H}]$ & & & & -0.90 & & 1 & -0.83 & & 1 & -0.86 & 0.04 \\
\hline$[\mathrm{MgI} / \mathrm{H}]$ & & & & -0.79 & & 1 & -0.75 & & 1 & -0.77 & 0.02 \\
\hline$[\mathrm{SiI} / \mathrm{H}]$ & -0.62 & 0.04 & 2 & -0.67 & 0.13 & 3 & -0.75 & 0.03 & 2 & -0.68 & 0.05 \\
\hline$[\mathrm{SiII} / \mathrm{H}]$ & & & & & & & & & & & \\
\hline$[\mathrm{SI} / \mathrm{H}]$ & & & & -0.54 & & 1 & & & & -0.54 & \\
\hline$[\mathrm{CaI} / \mathrm{H}]$ & -0.52 & 0.15 & 9 & -0.58 & 0.14 & 10 & -0.59 & 0.13 & 9 & -0.56 & 0.08 \\
\hline $\begin{array}{r}{[\mathrm{ScII} / \mathrm{H}]} \\
{[\mathrm{TiI} / \mathrm{H}]}\end{array}$ & -0.96 & 0.18 & 6 & -0.96 & 0.15 & 6 & -1.00 & 0.18 & 5 & -0.97 & 0.09 \\
\hline [TiII/H] & -0.80 & 0.21 & 4 & -0.78 & 0.24 & 3 & -0.80 & 0.21 & 3 & -0.79 & 0.11 \\
\hline$[\mathrm{CrI} / \mathrm{H}]$ & & & & -0.66 & & 1 & & & & -0.66 & \\
\hline$[\mathrm{CrII} / \mathrm{H}]$ & -0.76 & 0.16 & 4 & -0.77 & 0.13 & 4 & -0.77 & 0.21 & 3 & -0.77 & 0.09 \\
\hline$[\mathrm{MnI} / \mathrm{H}]$ & & & & & & & & & & & \\
\hline$[\mathrm{FeI} / \mathrm{H}]$ & -0.66 & 0.08 & 35 & -0.71 & 0.06 & 31 & -0.63 & 0.13 & 38 & -0.66 & 0.06 \\
\hline$[\mathrm{FeII} / \mathrm{H}]$ & -0.72 & 0.12 & 12 & -0.77 & 0.11 & 11 & -0.74 & 0.09 & 11 & -0.74 & 0.06 \\
\hline$[\mathrm{NiI} / \mathrm{H}]$ & -0.82 & & 1 & -0.87 & & 1 & & & & -0.84 & 0.03 \\
\hline$[\mathrm{YII} / \mathrm{H}]$ & -1.18 & & 1 & -1.00 & 0.27 & 2 & & & & -1.06 & 0.17 \\
\hline$[\mathrm{ZrII} / \mathrm{H}]$ & & & & & & & -0.73 & & 1 & -0.73 & \\
\hline [LaII/H] & & & & & & & & & & & \\
\hline$[\mathrm{NdII} / \mathrm{H}]$ & & & & & & & & & & & \\
\hline [EuII/H] & & & & & & & & & & & \\
\hline
\end{tabular}


B. Lemasle et al.: Detailed chemical composition of classical Cepheids in the Magellanic Clouds

Table B.2. Same as Table A.1 for HV 1328.

\begin{tabular}{|c|c|c|c|c|c|c|c|c|c|c|c|}
\hline HV 1328 & \multicolumn{3}{|c|}{ MJD $=54785.00779033$} & \multicolumn{3}{|c|}{ MJD $=54785.01762254$} & \multicolumn{3}{|c|}{$\mathrm{MJD}=54785.02744873$} & \multicolumn{2}{|c|}{ Abundance } \\
\hline Element & $\begin{array}{r}{[\mathrm{X} / \mathrm{H}]} \\
(\mathrm{dex})\end{array}$ & $\begin{array}{c}\sigma \\
(\mathrm{dex})\end{array}$ & $N$ & $\begin{array}{r}{[\mathrm{X} / \mathrm{H}]} \\
(\mathrm{dex})\end{array}$ & $\begin{array}{c}\sigma \\
(\mathrm{dex})\end{array}$ & $N$ & $\begin{array}{r}{[\mathrm{X} / \mathrm{H}]} \\
(\mathrm{dex})\end{array}$ & $\begin{array}{c}\sigma \\
(\mathrm{dex})\end{array}$ & $N$ & $\begin{array}{r}{[\mathrm{X} / \mathrm{H}]} \\
(\mathrm{dex})\end{array}$ & $\begin{array}{c}\sigma \\
(\mathrm{dex})\end{array}$ \\
\hline$[\mathrm{NaI} / \mathrm{H}]$ & -0.79 & & 1 & -0.80 & & 1 & -0.79 & & 1 & -0.79 & 0.01 \\
\hline$[\mathrm{MgI} / \mathrm{H}]$ & -0.70 & & 1 & -0.69 & & 1 & -0.71 & & 1 & -0.70 & 0.01 \\
\hline$[\mathrm{SiI} / \mathrm{H}]$ & -0.59 & 0.09 & 4 & -0.68 & 0.14 & 2 & -0.60 & 0.17 & 4 & -0.61 & 0.07 \\
\hline$[\mathrm{SiII} / \mathrm{H}]$ & -0.32 & & 1 & & & & & & & -0.32 & \\
\hline$[\mathrm{SI} / \mathrm{H}]$ & -0.55 & & 1 & -0.55 & & 1 & & & & -0.55 & \\
\hline$[\mathrm{CaI} / \mathrm{H}]$ & -0.58 & 0.07 & 10 & -0.61 & 0.06 & 10 & -0.64 & 0.05 & 10 & -0.61 & 0.04 \\
\hline$[\mathrm{ScII} / \mathrm{H}]$ & -0.66 & 0.17 & 5 & -0.68 & 0.14 & 6 & -0.71 & 0.16 & 4 & -0.68 & 0.08 \\
\hline$[\mathrm{Til} / \mathrm{H}]$ & -0.76 & & 1 & -0.79 & & 1 & -0.83 & & 1 & -0.79 & 0.02 \\
\hline$[\mathrm{TiII} / \mathrm{H}]$ & -0.74 & 0.05 & 4 & -0.67 & 0.08 & 4 & -0.67 & 0.13 & 5 & -0.69 & 0.06 \\
\hline$[\mathrm{CrI} / \mathrm{H}]$ & & & & & & & -0.69 & 0.08 & 2 & -0.69 & 0.08 \\
\hline$[\mathrm{CrII} / \mathrm{H}]$ & -0.54 & 0.11 & 2 & -0.62 & 0.15 & 4 & -0.63 & 0.15 & 4 & -0.61 & 0.08 \\
\hline$[\mathrm{MnI} / \mathrm{H}]$ & -0.85 & & 1 & -0.92 & & 1 & & & & -0.89 & 0.04 \\
\hline$[\mathrm{Fel} / \mathrm{H}]$ & -0.65 & 0.05 & 43 & -0.66 & 0.08 & 47 & -0.66 & 0.09 & 54 & -0.66 & 0.05 \\
\hline$[\mathrm{FeII} / \mathrm{H}]$ & -0.62 & 0.07 & 11 & -0.59 & 0.09 & 10 & -0.60 & 0.10 & 12 & -0.60 & 0.05 \\
\hline$[\mathrm{NiI} / \mathrm{H}]$ & -1.00 & 0.06 & 2 & -1.02 & 0.11 & 2 & -1.01 & 0.05 & 2 & -1.01 & 0.03 \\
\hline$[\mathrm{YII} / \mathrm{H}]$ & -0.89 & 0.16 & 2 & -0.87 & 0.14 & 2 & -0.94 & 0.11 & 2 & -0.90 & 0.06 \\
\hline$[\mathrm{ZrII} / \mathrm{H}]$ & -0.66 & & 1 & -0.66 & & 1 & -0.70 & & 1 & -0.67 & 0.01 \\
\hline$[\mathrm{LaII} / \mathrm{H}]$ & -0.21 & & 1 & & & & -0.38 & 0.18 & 2 & -0.32 & 0.12 \\
\hline$[\mathrm{NdII} / \mathrm{H}]$ & -0.50 & & 1 & -0.47 & & 1 & -0.49 & 0.04 & 2 & -0.49 & 0.02 \\
\hline$[\mathrm{EuII} / \mathrm{H}]$ & -0.04 & & 1 & & & & -0.08 & & 1 & -0.06 & 0.02 \\
\hline
\end{tabular}

Table B.3. Same as Table A.1 for HV 1333.

\begin{tabular}{|c|c|c|c|c|c|c|c|c|c|c|c|}
\hline \multirow{2}{*}{$\begin{array}{r}\text { HV } 1333 \\
\text { Element }\end{array}$} & \multicolumn{3}{|c|}{ MJD = 54 785.07912217 } & \multicolumn{3}{|c|}{ MJD $=54785.09358357$} & \multicolumn{3}{|c|}{ MJD = 54785.10804394} & \multicolumn{2}{|c|}{ Abundance } \\
\hline & $\begin{array}{r}{[\mathrm{X} / \mathrm{H}]} \\
(\mathrm{dex})\end{array}$ & $\begin{array}{c}\sigma \\
(\mathrm{dex})\end{array}$ & $N$ & $\begin{array}{r}{[\mathrm{X} / \mathrm{H}]} \\
(\mathrm{dex})\end{array}$ & $\begin{array}{c}\sigma \\
(\operatorname{dex})\end{array}$ & $N$ & $\begin{array}{r}{[\mathrm{X} / \mathrm{H}]} \\
(\mathrm{dex})\end{array}$ & $\begin{array}{c}\sigma \\
(\operatorname{dex})\end{array}$ & $N$ & $\begin{array}{r}{[\mathrm{X} / \mathrm{H}]} \\
(\mathrm{dex})\end{array}$ & $\begin{array}{c}\sigma \\
(\mathrm{dex})\end{array}$ \\
\hline$[\mathrm{NaI} / \mathrm{H}]$ & -0.95 & & 1 & -0.97 & & 1 & & & & -0.96 & 0.01 \\
\hline$[\mathrm{MgI} / \mathrm{H}]$ & -0.89 & & 1 & -0.67 & & 1 & -0.82 & & 1 & -0.79 & 0.06 \\
\hline$[\mathrm{SiI} / \mathrm{H}]$ & -0.88 & 0.10 & 4 & -0.68 & 0.13 & 3 & & & & -0.79 & 0.08 \\
\hline $\begin{array}{r}{[\mathrm{SiII} / \mathrm{H}]} \\
{[\mathrm{SI} / \mathrm{H}]}\end{array}$ & & & & & & & & & & & \\
\hline$[\mathrm{CaI} / \mathrm{H}]$ & -0.81 & 0.09 & 8 & -0.69 & 0.10 & 8 & -0.82 & 0.12 & 8 & -0.77 & 0.06 \\
\hline$[\mathrm{ScII} / \mathrm{H}]$ & -0.96 & 0.10 & 2 & -0.92 & 0.10 & 2 & -0.81 & & 1 & -0.91 & 0.05 \\
\hline$[\mathrm{TiI} / \mathrm{H}]$ & -1.00 & 0.08 & 2 & -0.91 & & 1 & -0.97 & 0.06 & 2 & -0.97 & 0.04 \\
\hline $\begin{array}{c}{[\mathrm{TiII} / \mathrm{H}]} \\
{[\mathrm{CrI} / \mathrm{H}]}\end{array}$ & -0.94 & 0.13 & 3 & -0.83 & 0.20 & 3 & -0.92 & 0.15 & 5 & -0.90 & 0.09 \\
\hline$[\mathrm{CrII} / \mathrm{H}]$ & -0.89 & 0.13 & 3 & -0.92 & 0.16 & 4 & -0.89 & 0.08 & 4 & -0.90 & 0.07 \\
\hline$[\mathrm{MnI} / \mathrm{H}]$ & -1.19 & 0.02 & 2 & -1.15 & & 1 & -1.20 & & 1 & -1.18 & 0.02 \\
\hline$[\mathrm{Fel} / \mathrm{H}]$ & -0.90 & 0.08 & 40 & -0.80 & 0.05 & 34 & -0.88 & 0.08 & 41 & -0.86 & 0.04 \\
\hline$[\mathrm{Fe} \mathrm{II} / \mathrm{H}]$ & -0.88 & 0.10 & 10 & -0.80 & 0.10 & 8 & -0.89 & 0.12 & 7 & -0.86 & 0.06 \\
\hline$[\mathrm{NiI} / \mathrm{H}]$ & -1.09 & 0.15 & 4 & -1.06 & 0.14 & 5 & -1.09 & 0.15 & 4 & -1.08 & 0.08 \\
\hline$[\mathrm{YII} / \mathrm{H}]$ & -1.10 & 0.07 & 5 & -1.01 & 0.08 & 4 & -1.10 & 0.09 & 3 & -1.07 & 0.04 \\
\hline$[\mathrm{ZrII} / \mathrm{H}]$ & -0.76 & & 1 & -0.57 & & 1 & -0.71 & & 1 & -0.68 & 0.06 \\
\hline [LaII/H] & -0.78 & 0.24 & 3 & -0.52 & 0.17 & 3 & -0.54 & 0.08 & 3 & -0.61 & 0.10 \\
\hline$[\mathrm{NdII} / \mathrm{H}]$ & -0.50 & 0.13 & 3 & -0.50 & 0.16 & 4 & -0.49 & 0.10 & 3 & -0.50 & 0.07 \\
\hline [EuII/H] & -0.36 & & 1 & -0.33 & & 1 & & & & $-\mathbf{0 . 3 4}$ & 0.01 \\
\hline
\end{tabular}


Table B.4. Same as Table A.1 for HV 1335.

\begin{tabular}{|c|c|c|c|c|c|c|c|c|c|c|c|}
\hline \multirow{2}{*}{$\begin{array}{r}\text { HV } 1335 \\
\text { Element }\end{array}$} & \multicolumn{3}{|c|}{$\mathrm{MJD}=54785.12765152$} & \multicolumn{3}{|c|}{$\mathrm{MJD}=54785.14326987$} & \multicolumn{3}{|c|}{ MJD = 54785.15889887} & \multicolumn{2}{|c|}{ Abundance } \\
\hline & $\begin{array}{r}{[\mathrm{X} / \mathrm{H}]} \\
(\mathrm{dex})\end{array}$ & $\begin{array}{c}\sigma \\
(\mathrm{dex})\end{array}$ & $N$ & $\begin{array}{r}{[\mathrm{X} / \mathrm{H}]} \\
(\mathrm{dex})\end{array}$ & $\begin{array}{c}\sigma \\
(\operatorname{dex})\end{array}$ & $N$ & $\begin{array}{r}{[\mathrm{X} / \mathrm{H}]} \\
(\mathrm{dex})\end{array}$ & $\begin{array}{c}\sigma \\
(\operatorname{dex})\end{array}$ & $N$ & $\begin{array}{r}{[\mathrm{X} / \mathrm{H}]} \\
(\mathrm{dex})\end{array}$ & $\begin{array}{c}\sigma \\
(\mathrm{dex})\end{array}$ \\
\hline$[\mathrm{NaI} / \mathrm{H}]$ & -0.99 & & 1 & -1.02 & & 1 & -0.87 & & 1 & -0.96 & 0.04 \\
\hline$[\mathrm{MgI} / \mathrm{H}]$ & -0.90 & & 1 & -0.78 & & 1 & -0.81 & & 1 & -0.83 & 0.04 \\
\hline$[\mathrm{SiI} / \mathrm{H}]$ & -0.92 & & 1 & -0.73 & & 2 & & & & -0.79 & 0.06 \\
\hline$[\mathrm{SiII} / \mathrm{H}]$ & -0.88 & & 1 & -1.02 & & 1 & -0.78 & & 1 & -0.89 & 0.07 \\
\hline$[\mathrm{SI} / \mathrm{H}]$ & -0.71 & & 1 & & & & & & & -0.71 & \\
\hline$[\mathrm{CaI} / \mathrm{H}]$ & -0.77 & 0.06 & 9 & -0.71 & 0.10 & 10 & -0.75 & 0.06 & 9 & -0.74 & 0.04 \\
\hline$[\mathrm{ScII} / \mathrm{H}]$ & -0.93 & 0.16 & 5 & -0.80 & 0.17 & 7 & -0.92 & 0.20 & 7 & -0.88 & 0.10 \\
\hline$[\mathrm{TiI} / \mathrm{H}]$ & -0.91 & 0.21 & 2 & -0.81 & & 1 & -1.05 & & 1 & -0.92 & 0.10 \\
\hline$[\mathrm{TiII} / \mathrm{H}]$ & -0.90 & 0.13 & 4 & -0.87 & 0.11 & 5 & -0.90 & 0.09 & 6 & -0.89 & 0.06 \\
\hline$[\mathrm{CrI} / \mathrm{H}]$ & -0.78 & & 1 & -0.65 & & 1 & & & & -0.72 & 0.07 \\
\hline$[\mathrm{CrII} / \mathrm{H}]$ & -0.85 & 0.22 & 3 & -0.83 & 0.10 & 4 & -0.87 & 0.16 & 3 & -0.85 & 0.08 \\
\hline$[\mathrm{MnI} / \mathrm{H}]$ & -1.06 & 0.03 & 2 & -1.08 & 0.02 & 2 & -1.17 & & 1 & -1.09 & 0.03 \\
\hline$[\mathrm{FeI} / \mathrm{H}]$ & -0.80 & 0.08 & 48 & -0.72 & 0.06 & 47 & -0.82 & 0.06 & 46 & -0.78 & 0.04 \\
\hline$[\mathrm{FeII} / \mathrm{H}]$ & -0.80 & 0.07 & 13 & -0.73 & 0.09 & 14 & -0.80 & 0.09 & 14 & -0.78 & 0.05 \\
\hline$[\mathrm{Nil} / \mathrm{H}]$ & -1.01 & 0.03 & 3 & -0.97 & 0.10 & 4 & -1.01 & 0.09 & 2 & -0.99 & 0.05 \\
\hline$[\mathrm{YII} / \mathrm{H}]$ & -1.00 & 0.10 & 4 & -0.93 & 0.09 & 3 & -1.02 & 0.18 & 4 & -0.99 & 0.07 \\
\hline$[\mathrm{ZrII} / \mathrm{H}]$ & -0.64 & & 1 & -0.58 & & 1 & -0.72 & & 1 & -0.65 & 0.04 \\
\hline$[\mathrm{LaII} / \mathrm{H}]$ & -0.58 & 0.13 & 4 & -0.57 & 0.11 & 4 & -0.67 & 0.12 & 4 & -0.61 & 0.07 \\
\hline$[\mathrm{NdII} / \mathrm{H}]$ & -0.51 & 0.05 & 3 & -0.49 & 0.04 & 3 & -0.49 & 0.06 & 3 & -0.50 & 0.03 \\
\hline [EuII/H] & -0.23 & 0.08 & 2 & -0.15 & & 1 & & & & -0.20 & 0.05 \\
\hline
\end{tabular}


B. Lemasle et al.: Detailed chemical composition of classical Cepheids in the Magellanic Clouds

\section{Appendix C: List of lines measured}

Table C.1. Wavelengths (in $\AA$ ) and atomic parameters of the spectral lines used in this study.

\begin{tabular}{|c|c|c|c|c|c|c|c|}
\hline $\begin{array}{l}\text { Wavelength } \\
(\AA)\end{array}$ & Element & $\begin{array}{l}\text { chi }_{\text {ex }} \\
(\mathrm{eV})\end{array}$ & $\log g f$ & $\begin{array}{l}\text { Wavelength } \\
\text { (A) }\end{array}$ & Element & $\begin{array}{l}\text { chi }_{\text {ex }} \\
(\mathrm{eV})\end{array}$ & $\log g f$ \\
\hline 4874.010 & Ti2 & 3.09 & -0.809 & 5627.497 & $\mathrm{Fe} 2$ & 3.39 & -4.10 \\
\hline 4892.859 & $\mathrm{Fe} 1$ & 4.22 & -1.292 & 5633.946 & $\mathrm{Fe} 1$ & 4.99 & -0.23 \\
\hline 4893.820 & $\mathrm{Fe} 2$ & 2.83 & -4.273 & 5638.262 & $\mathrm{Fe} 1$ & 4.22 & -0.77 \\
\hline 4917.230 & $\mathrm{Fe} 1$ & 4.19 & -1.089 & 5641.000 & $\mathrm{Sc} 2$ & 1.50 & -1.13 \\
\hline 4950.106 & $\mathrm{Fe} 1$ & 3.42 & -1.672 & 5641.434 & $\mathrm{Fe} 1$ & 4.26 & -1.08 \\
\hline 4959.115 & $\mathrm{Nd} 2$ & 0.06 & -0.806 & 5645.613 & Si1 & 4.93 & -2.04 \\
\hline 5005.157 & Ti2 & 1.57 & -2.737 & 5657.896 & $\mathrm{Sc} 2$ & 1.51 & -0.60 \\
\hline 5017.570 & Ni1 & 3.54 & -0.024 & 5658.816 & $\mathrm{Fe} 1$ & 3.40 & -0.81 \\
\hline 5044.211 & $\mathrm{Fe} 1$ & 2.85 & -2.045 & 5665.555 & Si1 & 4.92 & -1.94 \\
\hline 5084.089 & Ni1 & 3.68 & -0.088 & 5667.149 & $\mathrm{Sc} 2$ & 1.50 & -1.31 \\
\hline 5092.788 & $\mathrm{Nd} 2$ & 0.38 & -0.618 & 5669.042 & $\mathrm{Sc} 2$ & 1.50 & -1.20 \\
\hline 5112.270 & $\mathrm{Zr} 2$ & 1.66 & -0.856 & 5679.023 & $\mathrm{Fe} 1$ & 4.65 & -0.82 \\
\hline 5114.560 & La2 & 0.23 & -1.033 & 5682.633 & $\mathrm{Na} 1$ & 2.10 & -0.71 \\
\hline 5119.112 & Y2 & 0.99 & -1.369 & 5684.202 & $\mathrm{Sc} 2$ & 1.51 & -1.07 \\
\hline 5129.152 & Ti2 & 1.89 & -1.249 & 5686.530 & $\mathrm{Fe} 1$ & 4.55 & -0.66 \\
\hline 5130.586 & $\mathrm{Nd} 2$ & 1.30 & 0.450 & 5688.205 & $\mathrm{Na} 1$ & 2.10 & -0.40 \\
\hline 5133.688 & $\mathrm{Fe} 1$ & 4.18 & 0.148 & 5690.430 & Si1 & 4.93 & -1.77 \\
\hline 5153.402 & $\mathrm{Na} 1$ & 2.10 & -1.740 & 5693.620 & $\mathrm{Fe} 1$ & 4.96 & -2.59 \\
\hline 5155.125 & Nil & 3.90 & -0.560 & 5701.544 & $\mathrm{Fe} 1$ & 2.56 & -2.16 \\
\hline 5155.762 & Ni1 & 3.90 & 0.070 & 5705.464 & $\mathrm{Fe} 1$ & 4.30 & -1.35 \\
\hline 5159.058 & $\mathrm{Fe} 1$ & 4.28 & -0.828 & 5708.400 & Si1 & 4.95 & -1.37 \\
\hline 5176.559 & Ni1 & 3.90 & -0.300 & 5711.088 & Mg1 & 4.34 & -1.83 \\
\hline 5196.059 & $\mathrm{Fe} 1$ & 4.26 & -0.496 & 5717.833 & $\mathrm{Fe} 1$ & 4.28 & -1.03 \\
\hline 5210.385 & Ti1 & 0.05 & -0.835 & 5731.762 & $\mathrm{Fe} 1$ & 4.26 & -1.20 \\
\hline 5216.274 & $\mathrm{Fe} 1$ & 1.61 & -2.081 & 5732.860 & $\mathrm{Fe} 1$ & 4.10 & -2.90 \\
\hline 5217.389 & $\mathrm{Fe} 1$ & 3.21 & -1.121 & 5737.059 & V1 & 1.06 & -0.74 \\
\hline 5305.853 & $\mathrm{Cr} 2$ & 3.83 & -2.363 & 5740.858 & $\mathrm{Nd} 2$ & 1.16 & -0.53 \\
\hline 5307.361 & $\mathrm{Fe} 1$ & 1.61 & -2.911 & 5741.860 & $\mathrm{Fe} 1$ & 4.26 & -1.67 \\
\hline 5329.138 & $\mathrm{Cr} 1$ & 2.91 & -0.061 & 5752.032 & $\mathrm{Fe} 1$ & 4.55 & -1.18 \\
\hline 5329.990 & $\mathrm{Fe} 1$ & 4.08 & -1.198 & 5753.120 & $\mathrm{Fe} 1$ & 4.26 & -0.69 \\
\hline 5334.869 & $\mathrm{Cr} 2$ & 4.07 & -1.617 & 5853.668 & $\mathrm{Ba} 2$ & 0.60 & -0.91 \\
\hline 5336.771 & Ti2 & 1.58 & -1.638 & 5859.586 & $\mathrm{Fe} 1$ & 4.55 & -0.42 \\
\hline 5345.796 & $\mathrm{Cr} 1$ & 1.00 & -0.950 & 5862.357 & $\mathrm{Fe} 1$ & 4.55 & -0.13 \\
\hline 5349.465 & $\mathrm{Ca} 1$ & 2.71 & -0.311 & 5866.451 & Ti1 & 1.07 & -0.78 \\
\hline 5353.370 & $\mathrm{Fe} 1$ & 4.10 & -0.840 & 5899.300 & Ti1 & 1.05 & -1.17 \\
\hline 5369.961 & $\mathrm{Fe} 1$ & 4.37 & 0.537 & 5905.671 & $\mathrm{Fe} 1$ & 4.65 & -0.69 \\
\hline 5373.709 & $\mathrm{Fe} 1$ & 4.47 & -0.767 & 5909.973 & $\mathrm{Fe} 1$ & 3.21 & -2.59 \\
\hline 5379.574 & $\mathrm{Fe} 1$ & 3.69 & -1.519 & 5916.247 & $\mathrm{Fe} 1$ & 2.45 & -2.91 \\
\hline 5381.015 & Ti2 & 1.57 & -1.977 & 5927.789 & $\mathrm{Fe} 1$ & 4.65 & -0.99 \\
\hline 5383.369 & $\mathrm{Fe} 1$ & 4.31 & 0.641 & 5930.179 & $\mathrm{Fe} 1$ & 4.65 & -0.23 \\
\hline 5398.279 & $\mathrm{Fe} 1$ & 4.44 & -0.634 & 5934.655 & $\mathrm{Fe} 1$ & 3.93 & -1.07 \\
\hline 5402.774 & Y2 & 1.84 & -0.634 & 5948.541 & Si1 & 5.08 & -1.13 \\
\hline 5410.910 & $\mathrm{Fe} 1$ & 4.47 & 0.407 & 5956.694 & $\mathrm{Fe} 1$ & 0.86 & -4.55 \\
\hline
\end{tabular}


Table C.1. continued.

\begin{tabular}{|c|c|c|c|c|c|c|c|}
\hline $\begin{array}{l}\text { Wavelength } \\
(\AA)\end{array}$ & Element & $\begin{array}{l}\text { chi }_{\mathrm{ex}} \\
(\mathrm{eV})\end{array}$ & $\log g f$ & $\begin{array}{l}\text { Wavelength } \\
\text { (A) }\end{array}$ & Element & $\begin{array}{l}\text { chi }_{\text {ex }} \\
(\mathrm{eV})\end{array}$ & $\log g f$ \\
\hline 5414.073 & $\mathrm{Fe} 2$ & 3.22 & -3.582 & 5976.777 & $\mathrm{Fe} 1$ & 3.94 & -1.24 \\
\hline 5420.922 & $\mathrm{Cr} 2$ & 3.76 & -2.466 & 5983.680 & $\mathrm{Fe} 1$ & 4.55 & -1.47 \\
\hline 5425.254 & $\mathrm{Fe} 1$ & 1.01 & -2.121 & 5984.815 & $\mathrm{Fe} 1$ & 4.73 & -0.20 \\
\hline 5445.042 & $\mathrm{Fe} 1$ & 4.39 & -0.029 & 5987.065 & $\mathrm{Fe} 1$ & 4.80 & -0.43 \\
\hline 5454.090 & Ti2 & 1.57 & -3.547 & 5991.376 & $\mathrm{Fe} 2$ & 3.15 & -3.65 \\
\hline 5462.960 & $\mathrm{Fe} 1$ & 4.47 & -0.047 & 6003.011 & $\mathrm{Fe} 1$ & 3.88 & -1.12 \\
\hline 5463.276 & $\mathrm{Fe} 1$ & 4.43 & 0.073 & 6007.960 & $\mathrm{Fe} 1$ & 4.65 & -0.60 \\
\hline 5501.465 & $\mathrm{Fe} 1$ & 0.96 & -3.056 & 6008.556 & $\mathrm{Fe} 1$ & 3.88 & -0.99 \\
\hline 5502.067 & $\mathrm{Cr} 2$ & 4.17 & -2.097 & 6013.513 & Mn1 & 3.07 & -0.35 \\
\hline 5509.895 & $\mathrm{Y} 2$ & 0.99 & -0.959 & 6016.673 & Mn1 & 3.07 & -0.18 \\
\hline 5526.790 & $\mathrm{Sc} 2$ & 1.77 & 0.027 & 6020.169 & $\mathrm{Fe} 1$ & 4.61 & -0.27 \\
\hline 5528.405 & Mg1 & 4.35 & -0.625 & 6021.819 & Mn1 & 3.08 & -0.05 \\
\hline 5572.842 & $\mathrm{Fe} 1$ & 3.40 & -0.270 & 6024.058 & $\mathrm{Fe} 1$ & 4.55 & -0.12 \\
\hline 5576.089 & $\mathrm{Fe} 1$ & 3.43 & -0.903 & 6027.051 & $\mathrm{Fe} 1$ & 4.08 & -1.09 \\
\hline 5581.965 & $\mathrm{Ca} 1$ & 2.52 & -0.552 & 6055.990 & $\mathrm{Fe} 1$ & 4.73 & -0.46 \\
\hline 5590.114 & $\mathrm{Ca} 1$ & 2.52 & -0.572 & 6065.482 & $\mathrm{Fe} 1$ & 2.61 & -1.47 \\
\hline 5591.370 & $\mathrm{Fe} 2$ & 3.27 & -4.597 & 6078.491 & $\mathrm{Fe} 1$ & 4.80 & -0.32 \\
\hline 5601.277 & $\mathrm{Ca} 1$ & 2.53 & -0.523 & 6079.008 & $\mathrm{Fe} 1$ & 4.65 & -1.02 \\
\hline 6082.710 & $\mathrm{Fe} 1$ & 2.22 & -3.57 & 6335.330 & $\mathrm{Fe} 1$ & 2.20 & -2.18 \\
\hline 6084.111 & $\mathrm{Fe} 2$ & 3.20 & -3.88 & 6336.823 & $\mathrm{Fe} 1$ & 3.69 & -0.86 \\
\hline 6085.270 & $\mathrm{Fe} 1$ & 2.76 & -2.86 & 6344.148 & $\mathrm{Fe} 1$ & 2.43 & -2.90 \\
\hline 6091.919 & Sil & 5.87 & -1.47 & 6347.109 & $\mathrm{Si} 2$ & 8.12 & 0.17 \\
\hline 6096.664 & $\mathrm{Fe} 1$ & 3.98 & -1.83 & 6355.028 & $\mathrm{Fe} 1$ & 2.85 & -2.32 \\
\hline 6102.180 & $\mathrm{Fe} 1$ & 4.84 & -0.10 & 6358.697 & $\mathrm{Fe} 1$ & 0.86 & -4.47 \\
\hline 6102.723 & $\mathrm{Ca} 1$ & 1.88 & -0.79 & 6362.338 & $\mathrm{Zn} 1$ & 5.80 & 0.14 \\
\hline 6108.107 & Ni1 & 1.68 & -2.44 & 6369.462 & $\mathrm{Fe} 2$ & 2.89 & -4.11 \\
\hline 6113.322 & $\mathrm{Fe} 2$ & 3.22 & -4.23 & 6380.743 & $\mathrm{Fe} 1$ & 4.19 & -1.37 \\
\hline 6122.217 & $\mathrm{Ca} 1$ & 1.89 & -0.32 & 6390.477 & $\mathrm{La} 2$ & 0.32 & -1.41 \\
\hline 6127.906 & $\mathrm{Fe} 1$ & 4.14 & -1.40 & 6393.600 & $\mathrm{Fe} 1$ & 2.43 & -1.50 \\
\hline 6141.713 & $\mathrm{Ba} 2$ & 0.70 & -0.03 & 6407.251 & $\mathrm{Fe} 2$ & 3.89 & -3.85 \\
\hline 6149.258 & $\mathrm{Fe} 2$ & 3.89 & -2.84 & 6408.018 & $\mathrm{Fe} 1$ & 3.69 & -1.02 \\
\hline 6151.617 & $\mathrm{Fe} 1$ & 2.18 & -3.31 & 6411.648 & $\mathrm{Fe} 1$ & 3.65 & -0.66 \\
\hline 6155.134 & Sil & 5.62 & -0.75 & 6414.980 & Si1 & 5.87 & -1.04 \\
\hline 6157.728 & $\mathrm{Fe} 1$ & 4.08 & -1.16 & 6416.919 & $\mathrm{Fe} 2$ & 3.89 & -2.88 \\
\hline 6160.747 & $\mathrm{Na} 1$ & 2.10 & -1.25 & 6419.980 & $\mathrm{Fe} 1$ & 4.73 & -0.17 \\
\hline 6161.297 & $\mathrm{Ca} 1$ & 2.52 & -1.27 & 6421.350 & $\mathrm{Fe} 1$ & 2.28 & -2.02 \\
\hline 6162.173 & $\mathrm{Ca} 1$ & 1.90 & -0.09 & 6430.845 & $\mathrm{Fe} 1$ & 2.18 & -1.98 \\
\hline 6165.360 & $\mathrm{Fe} 1$ & 4.14 & -1.47 & 6432.680 & $\mathrm{Fe} 2$ & 2.89 & -3.57 \\
\hline 6166.439 & $\mathrm{Ca} 1$ & 2.52 & -1.14 & 6433.457 & Si1 & 5.96 & -2.06 \\
\hline 6169.042 & $\mathrm{Ca} 1$ & 2.52 & -0.80 & 6437.640 & Eu2 & 1.32 & -0.32 \\
\hline 6169.563 & $\mathrm{Ca} 1$ & 2.53 & -0.48 & 6439.075 & Ca1 & 2.52 & 0.39 \\
\hline 6170.506 & $\mathrm{Fe} 1$ & 4.79 & -0.44 & 6449.808 & Ca1 & 2.52 & -0.50 \\
\hline 6173.334 & $\mathrm{Fe} 1$ & 2.22 & -2.88 & 6455.598 & Ca1 & 2.52 & -1.29 \\
\hline 6175.360 & Ni1 & 4.09 & -0.39 & 6462.567 & Ca1 & 2.52 & 0.26 \\
\hline
\end{tabular}


B. Lemasle et al.: Detailed chemical composition of classical Cepheids in the Magellanic Clouds

Table C.1. continued.

\begin{tabular}{|c|c|c|c|c|c|c|c|}
\hline $\begin{array}{c}\text { Wavelength } \\
(\AA)\end{array}$ & Element & $\begin{array}{l}\text { chi }_{\text {ex }} \\
(\mathrm{eV})\end{array}$ & $\log g f$ & $\begin{array}{c}\text { Wavelength } \\
(\AA)\end{array}$ & Element & $\begin{array}{l}\mathrm{chi}_{\mathrm{ex}} \\
(\mathrm{eV})\end{array}$ & $\log g f$ \\
\hline 6176.807 & Ni1 & 4.09 & -0.26 & 6471.662 & Cal & 2.53 & -0.69 \\
\hline 6180.203 & $\mathrm{Fe} 1$ & 2.73 & -2.62 & 6481.870 & $\mathrm{Fe} 1$ & 2.28 & -2.99 \\
\hline 6187.989 & $\mathrm{Fe} 1$ & 3.94 & -1.62 & 6482.796 & Ni1 & 1.93 & -2.63 \\
\hline 6191.558 & $\mathrm{Fe} 1$ & 2.43 & -1.42 & 6491.561 & Ti2 & 2.06 & -1.94 \\
\hline 6200.313 & $\mathrm{Fe} 1$ & 2.61 & -2.40 & 6493.781 & Ca1 & 2.52 & -0.11 \\
\hline 6213.430 & $\mathrm{Fe} 1$ & 2.22 & -2.48 & 6494.980 & $\mathrm{Fe} 1$ & 2.40 & -1.26 \\
\hline 6215.150 & $\mathrm{Fe} 1$ & 4.19 & -1.14 & 6496.897 & $\mathrm{Ba} 2$ & 0.60 & -0.41 \\
\hline 6216.354 & V1 & 0.28 & -1.29 & 6498.938 & $\mathrm{Fe} 1$ & 0.96 & -4.69 \\
\hline 6219.281 & $\mathrm{Fe} 1$ & 2.20 & -2.43 & 6499.650 & $\mathrm{Ca} 1$ & 2.52 & -0.82 \\
\hline 6229.226 & $\mathrm{Fe} 1$ & 2.85 & -2.80 & 6516.080 & $\mathrm{Fe} 2$ & 2.89 & -3.31 \\
\hline 6230.722 & $\mathrm{Fe} 1$ & 2.56 & -1.28 & 6518.366 & $\mathrm{Fe} 1$ & 2.83 & -2.37 \\
\hline 6232.640 & $\mathrm{Fe} 1$ & 3.65 & -1.22 & 6559.588 & Ti2 & 2.05 & -2.17 \\
\hline 6237.319 & Si1 & 5.61 & -0.98 & 6569.214 & $\mathrm{Fe} 1$ & 4.73 & -0.38 \\
\hline 6238.392 & $\mathrm{Fe} 2$ & 3.89 & -2.60 & 6572.779 & $\mathrm{Ca} 1$ & 0.00 & -4.24 \\
\hline 6239.370 & $\mathrm{Fe} 2$ & 2.81 & -4.76 & 6586.308 & Ni1 & 1.95 & -2.75 \\
\hline 6239.953 & $\mathrm{Fe} 2$ & 3.89 & -3.57 & 6592.913 & $\mathrm{Fe} 1$ & 2.73 & -1.47 \\
\hline 6240.646 & $\mathrm{Fe} 1$ & 2.22 & -3.20 & 6593.870 & $\mathrm{Fe} 1$ & 2.43 & -2.39 \\
\hline 6243.815 & Si1 & 5.62 & -1.24 & 6604.601 & $\mathrm{Sc} 2$ & 1.36 & -1.31 \\
\hline 6244.466 & Sil & 5.62 & -1.09 & 6606.949 & $\mathrm{Ti} 2$ & 2.06 & -2.80 \\
\hline 6245.637 & $\mathrm{Sc} 2$ & 1.51 & -1.02 & 6609.110 & $\mathrm{Fe} 1$ & 2.56 & -2.68 \\
\hline 6246.318 & $\mathrm{Fe} 1$ & 3.60 & -0.80 & 6613.733 & $\mathrm{Y} 2$ & 1.75 & -0.85 \\
\hline 6247.557 & $\mathrm{Fe} 2$ & 3.89 & -2.43 & 6645.064 & $\mathrm{Eu} 2$ & 1.38 & 0.12 \\
\hline 6252.555 & $\mathrm{Fe} 1$ & 2.40 & -1.72 & 6677.990 & $\mathrm{Fe} 1$ & 2.69 & -1.37 \\
\hline 6254.258 & $\mathrm{Fe} 1$ & 2.28 & -2.43 & 6680.133 & $\mathrm{Ti} 2$ & 3.09 & -1.79 \\
\hline 6258.100 & Ti1 & 1.44 & -0.30 & 6680.140 & $\mathrm{Cr} 1$ & 4.16 & -0.39 \\
\hline 6261.100 & Ti1 & 1.43 & -0.42 & 6703.566 & $\mathrm{Fe} 1$ & 2.76 & -3.06 \\
\hline 6262.290 & $\mathrm{La} 2$ & 0.40 & -1.22 & 6715.410 & $\mathrm{Fe} 1$ & 4.61 & -1.36 \\
\hline 6265.132 & $\mathrm{Fe} 1$ & 2.18 & -2.54 & 6717.681 & $\mathrm{Ca} 1$ & 2.71 & -0.52 \\
\hline 6271.278 & $\mathrm{Fe} 1$ & 3.33 & -2.70 & 6721.848 & Sil & 5.86 & -1.52 \\
\hline 6279.753 & $\mathrm{Sc} 2$ & 1.50 & -1.25 & 6726.666 & $\mathrm{Fe} 1$ & 4.61 & -1.13 \\
\hline 6290.965 & $\mathrm{Fe} 1$ & 4.73 & -0.77 & 6740.080 & $\mathrm{Nd} 2$ & 0.06 & -1.53 \\
\hline 6297.793 & $\mathrm{Fe} 1$ & 2.22 & -2.70 & 6748.837 & $\mathrm{~S} 1$ & 7.87 & -0.64 \\
\hline 6301.500 & $\mathrm{Fe} 1$ & 3.65 & -0.72 & 6750.152 & $\mathrm{Fe} 1$ & 2.42 & -2.60 \\
\hline 6318.018 & $\mathrm{Fe} 1$ & 2.45 & -1.80 & 6757.171 & $\mathrm{~S} 1$ & 7.87 & -0.24 \\
\hline 6318.717 & $\operatorname{Mg} 1$ & 5.11 & -2.10 & 6767.768 & Ni1 & 1.83 & -2.17 \\
\hline 6320.410 & $\mathrm{La} 2$ & 0.17 & -1.33 & 6772.313 & Nil & 3.66 & -0.80 \\
\hline 6320.851 & $\mathrm{Sc} 2$ & 1.50 & -1.82 & 6774.270 & $\mathrm{La} 2$ & 0.13 & -1.71 \\
\hline 6322.685 & $\mathrm{Fe} 1$ & 2.59 & -2.45 & 6795.414 & $\mathrm{Y} 2$ & 1.74 & -1.03 \\
\hline 6327.593 & Nil & 1.68 & -3.15 & & & & \\
\hline
\end{tabular}




\section{Appendix D: Curves of growth}

The curve of growth is a graph showing how the equivalent width (EW) of an absorption line varies with the abundance $(A)$ of the atoms/ions producing the line. When there are few atoms, the line profile is dominated by Doppler broadening and $E W \propto A$. When the line reaches saturation, $E W \propto \sqrt{\ln A}$. When saturation increases, the line profile becomes dominated by the wings due to collisional broadening and $E W \propto \sqrt{A}$.

For weak lines it can be shown that $\log \left(\frac{W_{\lambda}}{\lambda}\right)$ varies linearly with $\log \left(\alpha_{*} g f\right)$. The $\Gamma_{*}$ quantity, defined as $\log \left(\frac{W_{\lambda}}{\lambda}\right)=\log \left(\alpha_{*} g f\right)+$ $\log \Gamma_{*}$ can be computed for each individual line as a function of the atmosphere model, the element considered and its ionization stage, the line excitation potential and so on. As the value of the stellar abundance $\alpha_{*}$ is the unknown one wants to determine, the abundance of the same element in the Sun $\alpha_{\odot}$ is used instead. The experimental curves of growth will then be shifted from the theoretical curves of growth by a factor $\log \left(\alpha_{*}\right)-\log \left(\alpha_{\odot}\right)$, which is by definition the abundance ratio [X/H] of the considered element X.

The experimental curve of growth is traced using many lines from the same element (e.g., Fe I) with a wide range of excitation potentials and oscillator strengths that lead to a wide range of equivalent widths. The curves of growth plotted below show the theoretical curves of growths computed for a line at $\lambda=5000 \AA$ with $\chi$ ex $=3$ and using the atmospheric parameters derived for the star in the corresponding phase. 
B. Lemasle et al.: Detailed chemical composition of classical Cepheids in the Magellanic Clouds
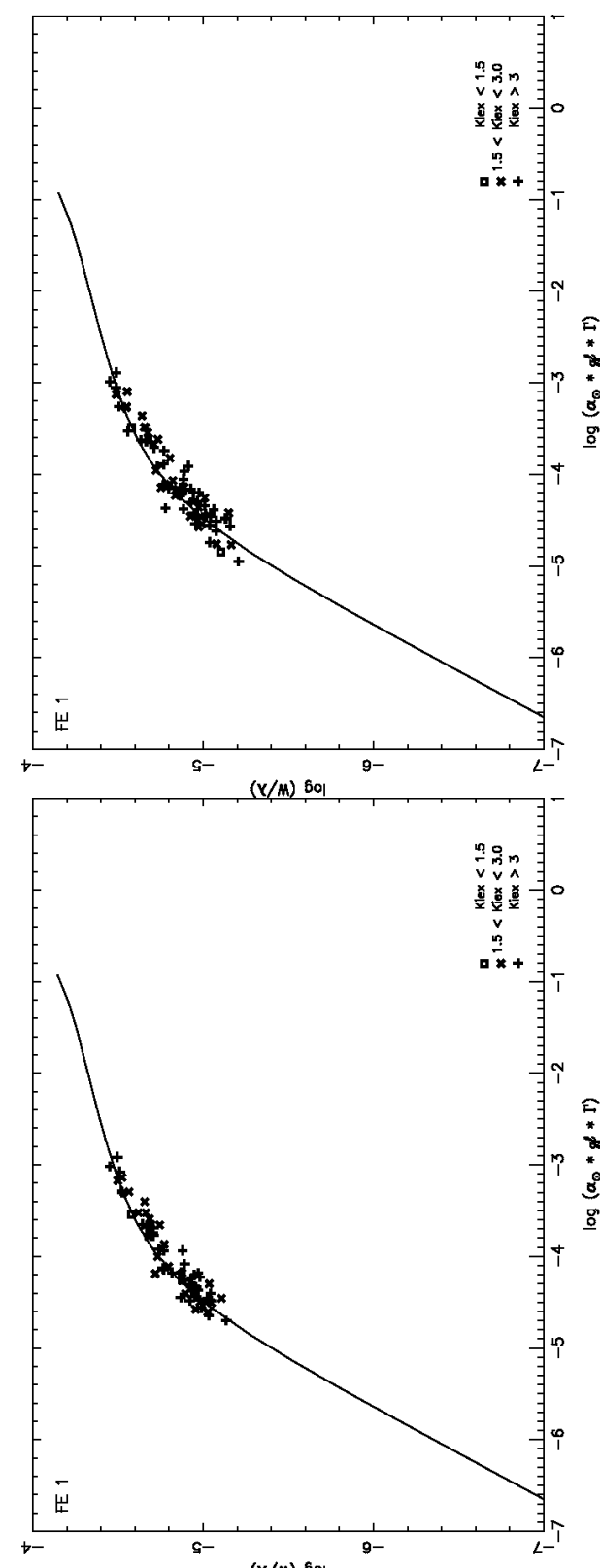

e

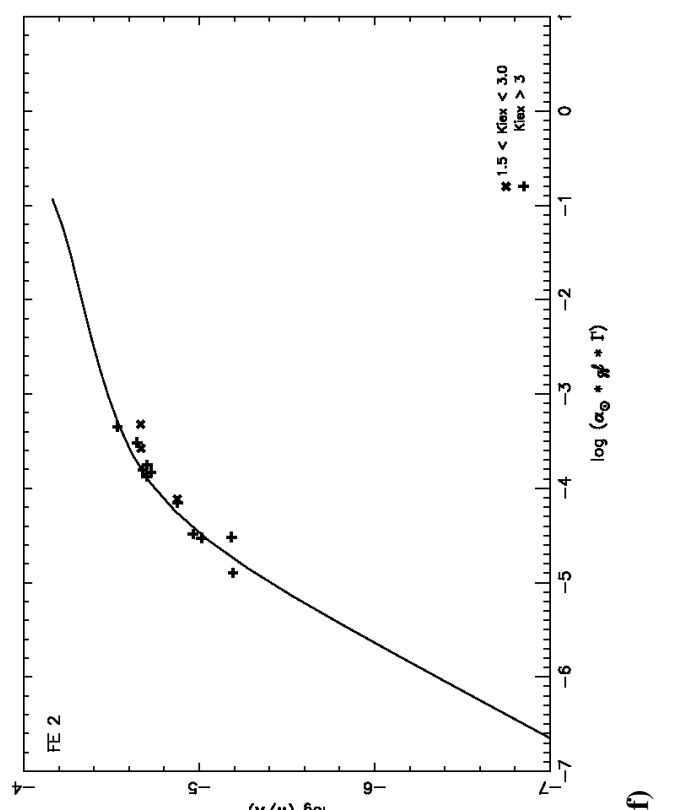

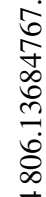

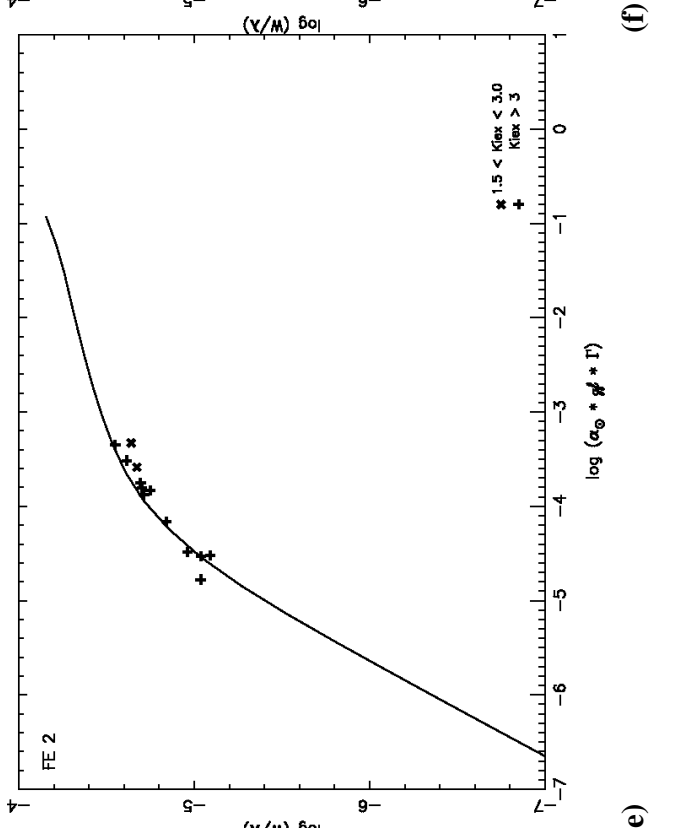

$\hat{\varepsilon}$

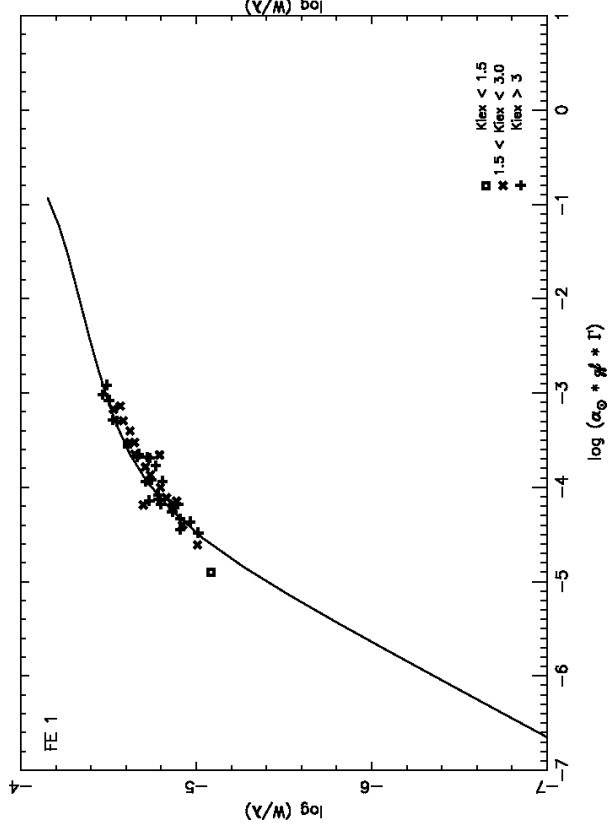

ङ

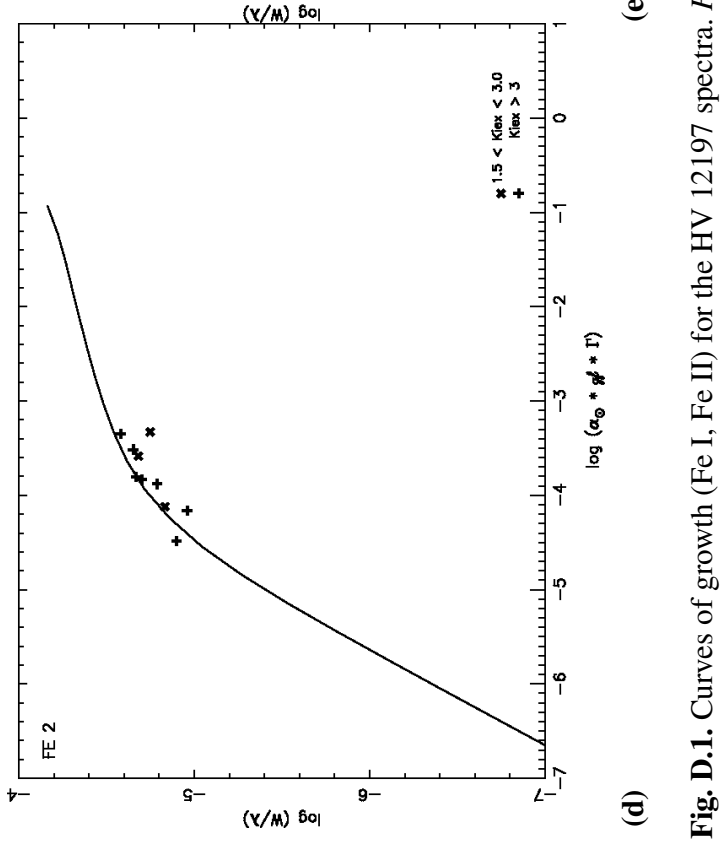

A85, page 27 of 36 
A\&A 608, A85 (2017)
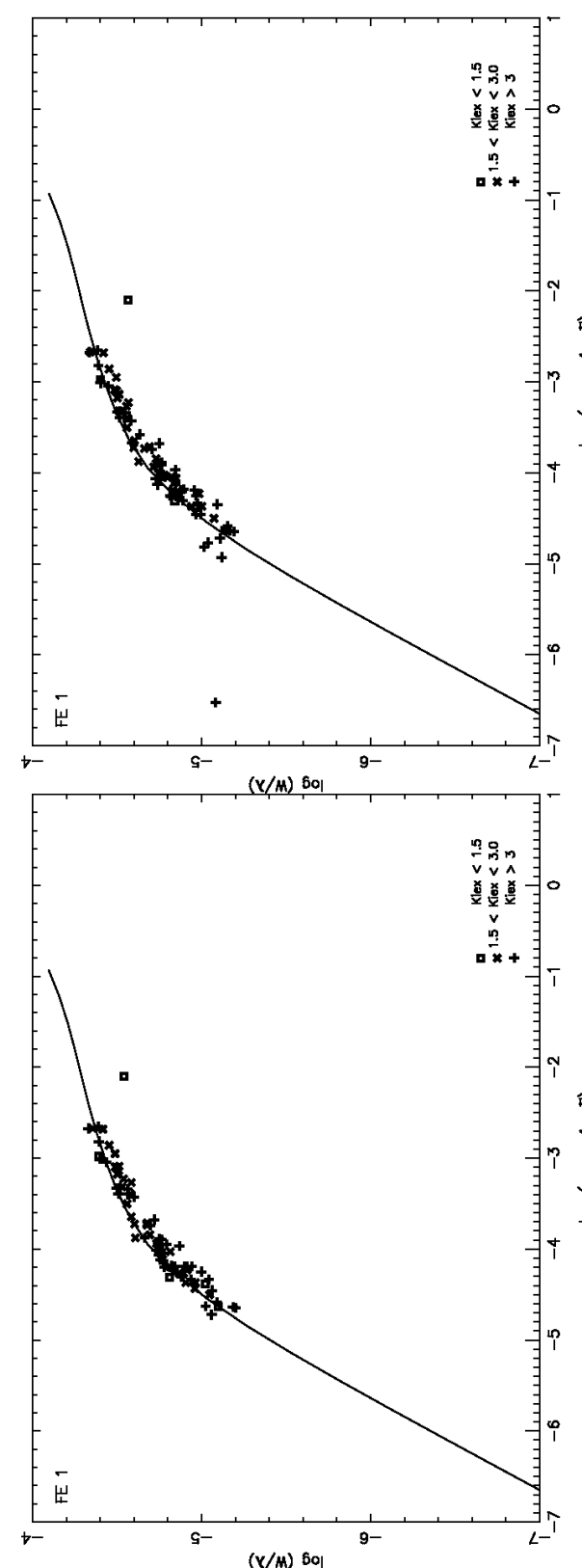

e

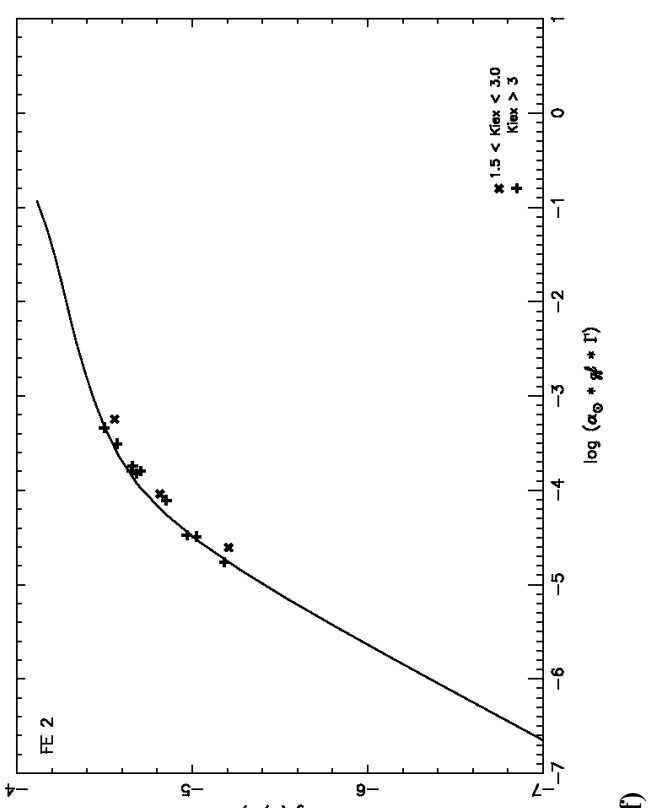

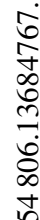
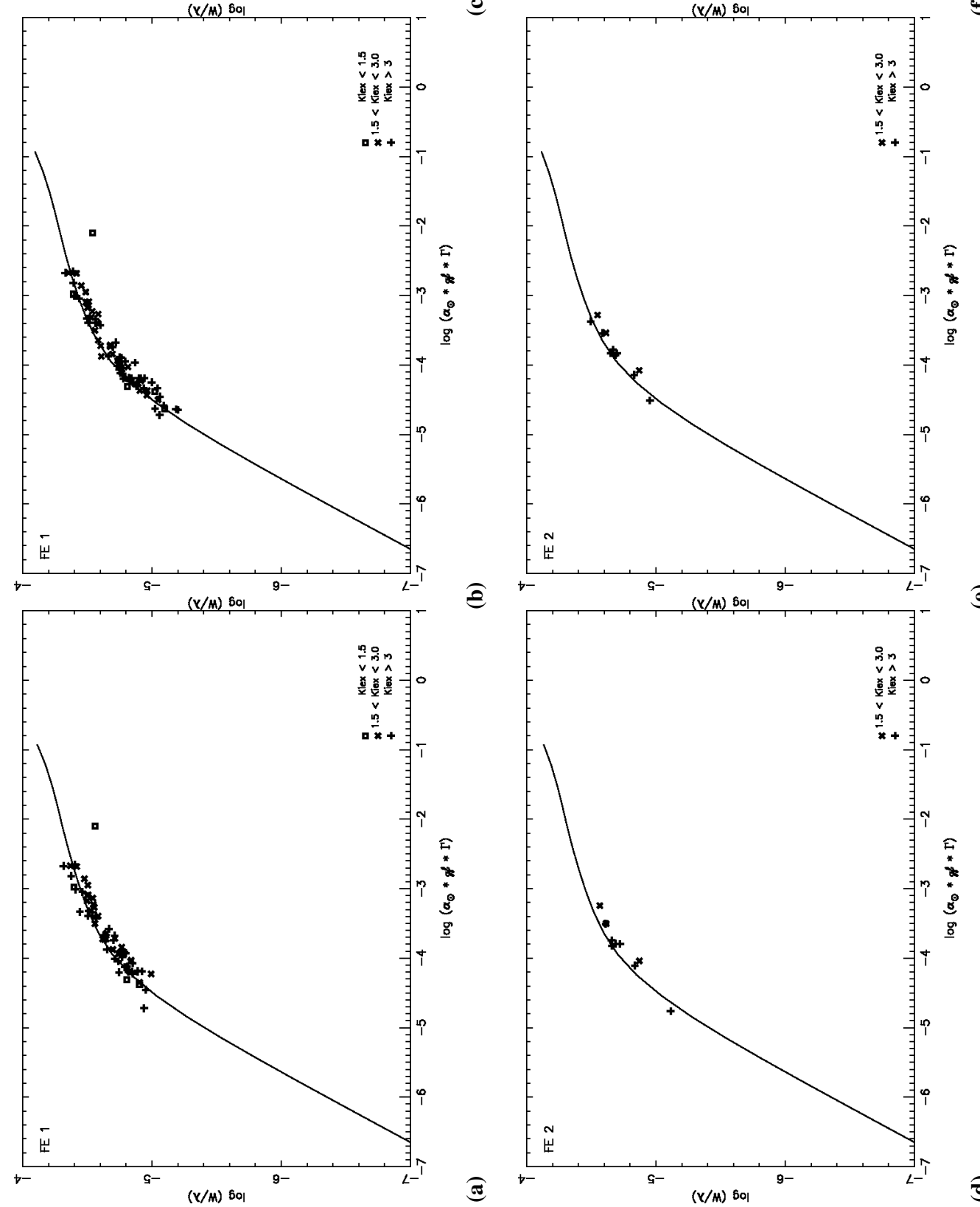

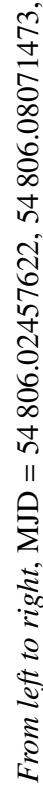

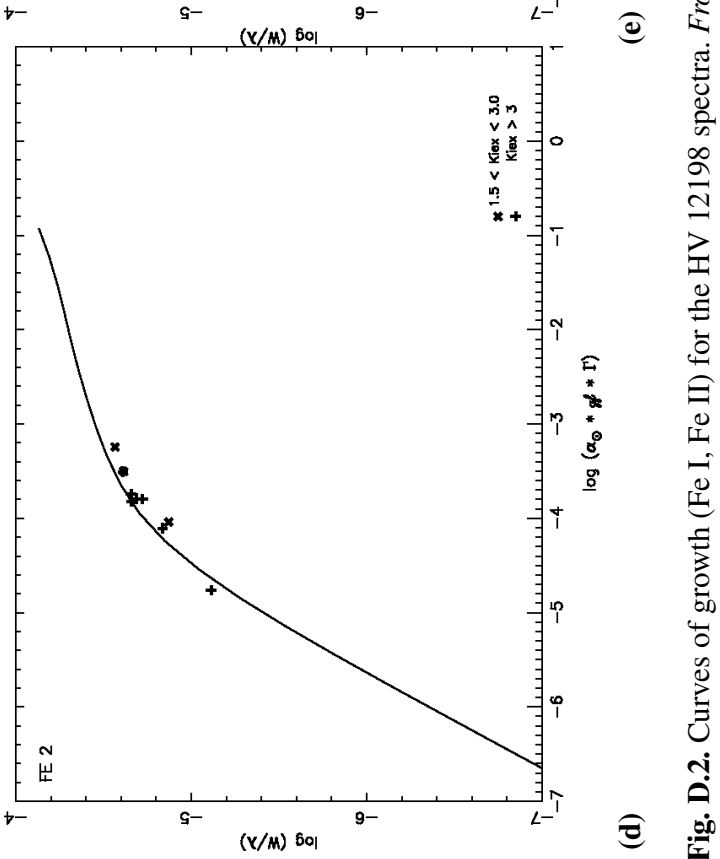


B. Lemasle et al.: Detailed chemical composition of classical Cepheids in the Magellanic Clouds
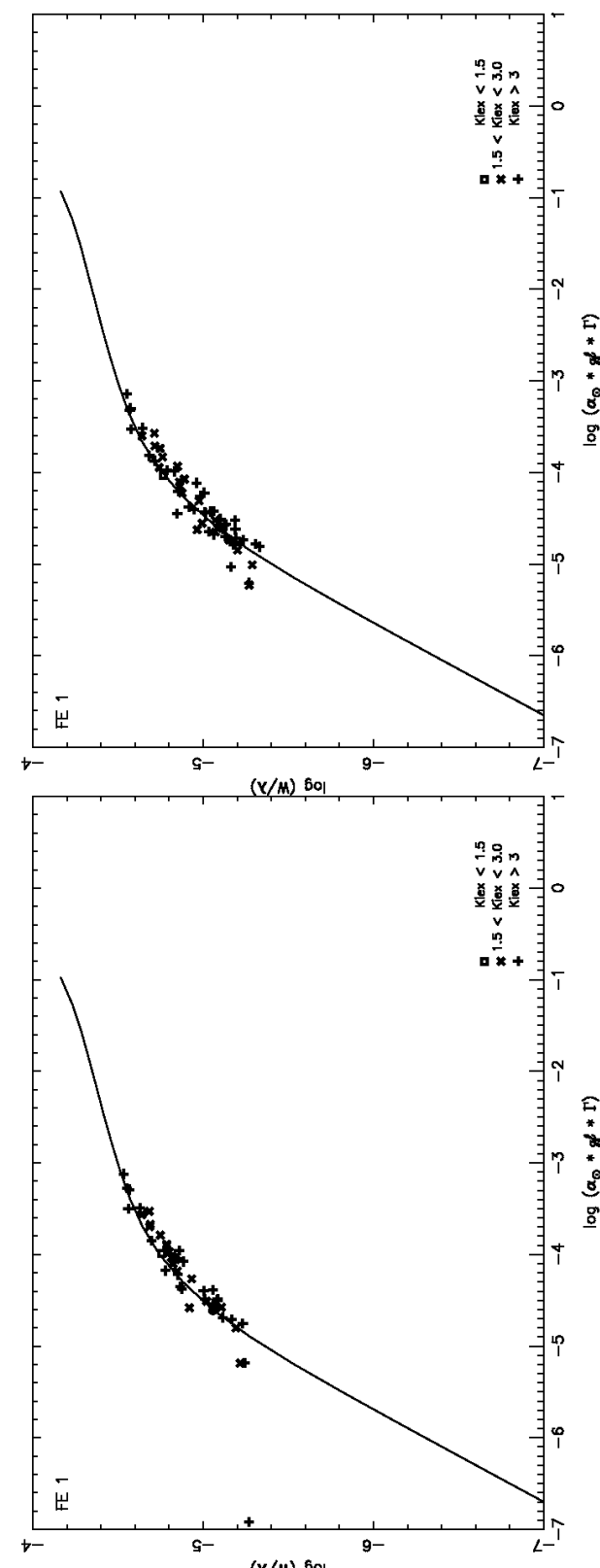

e

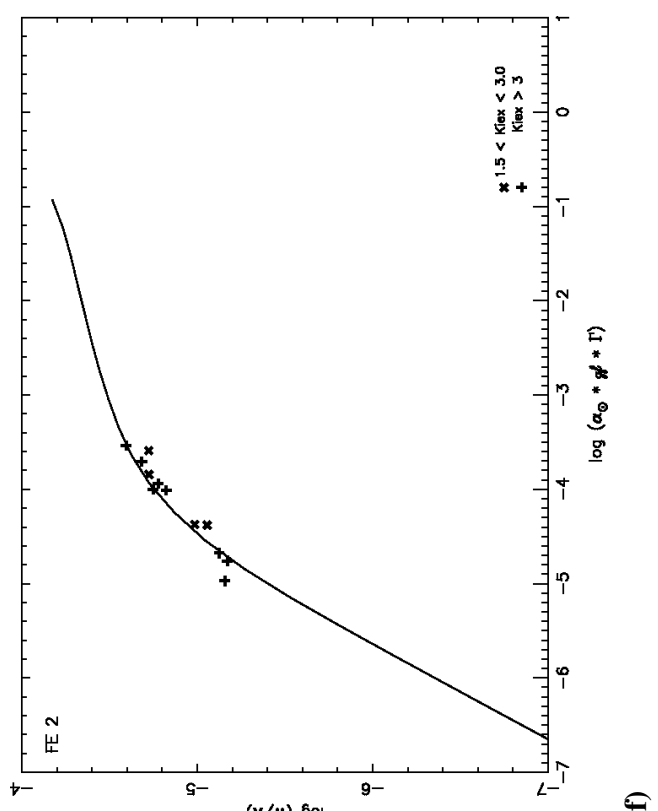

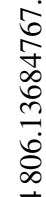

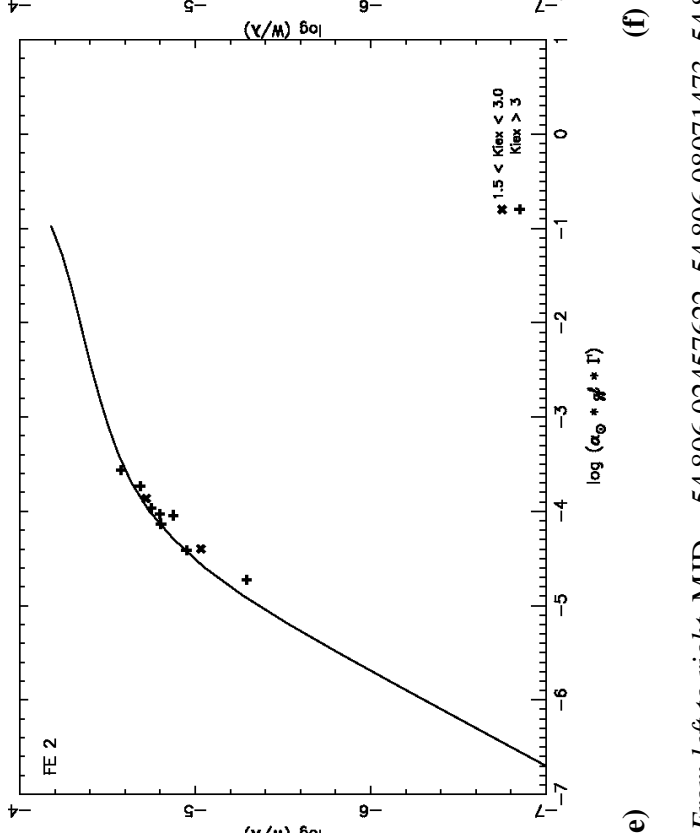

$\hat{e}$

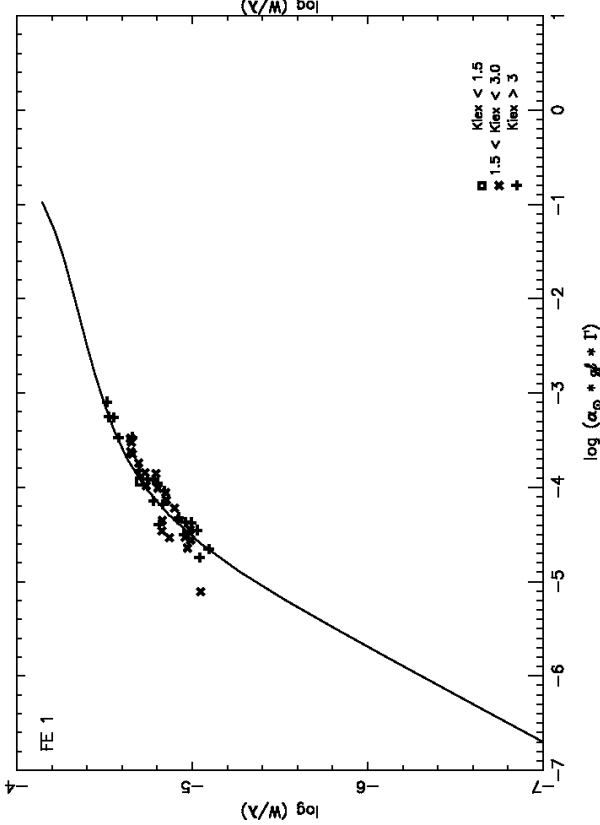

ङ

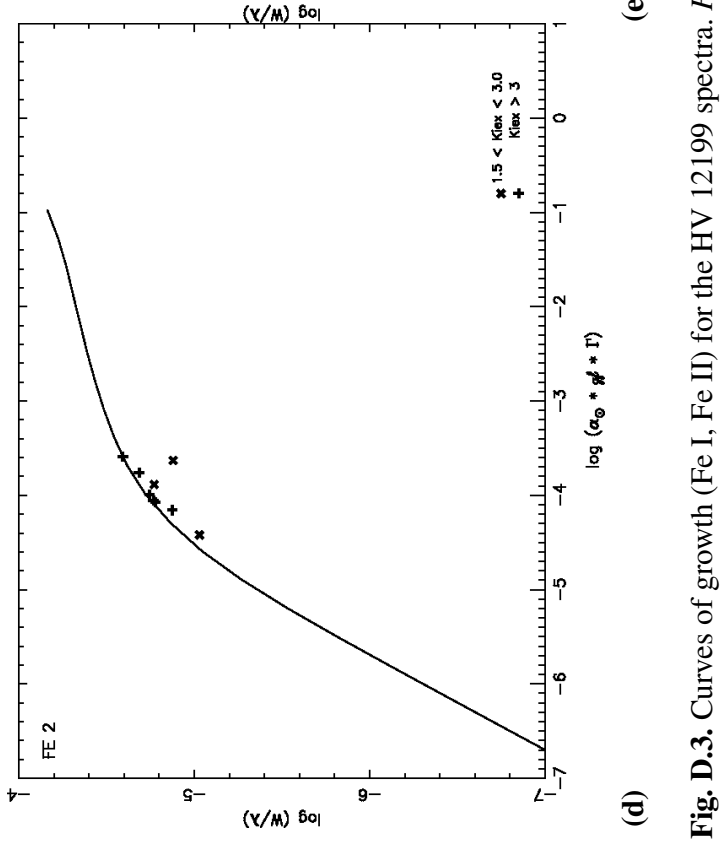

A85, page 29 of 36 
A\&A 608, A85 (2017)
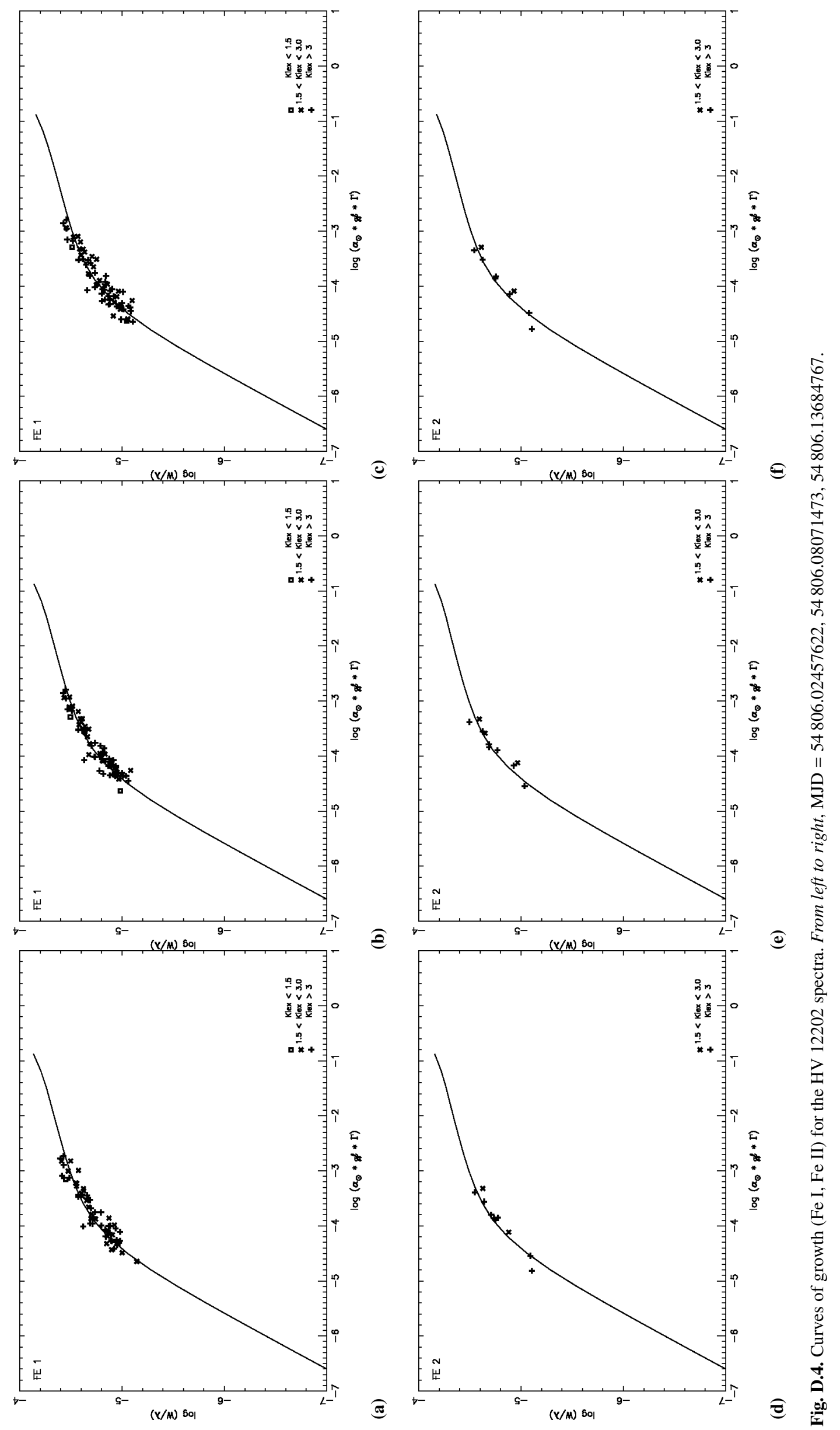
B. Lemasle et al.: Detailed chemical composition of classical Cepheids in the Magellanic Clouds
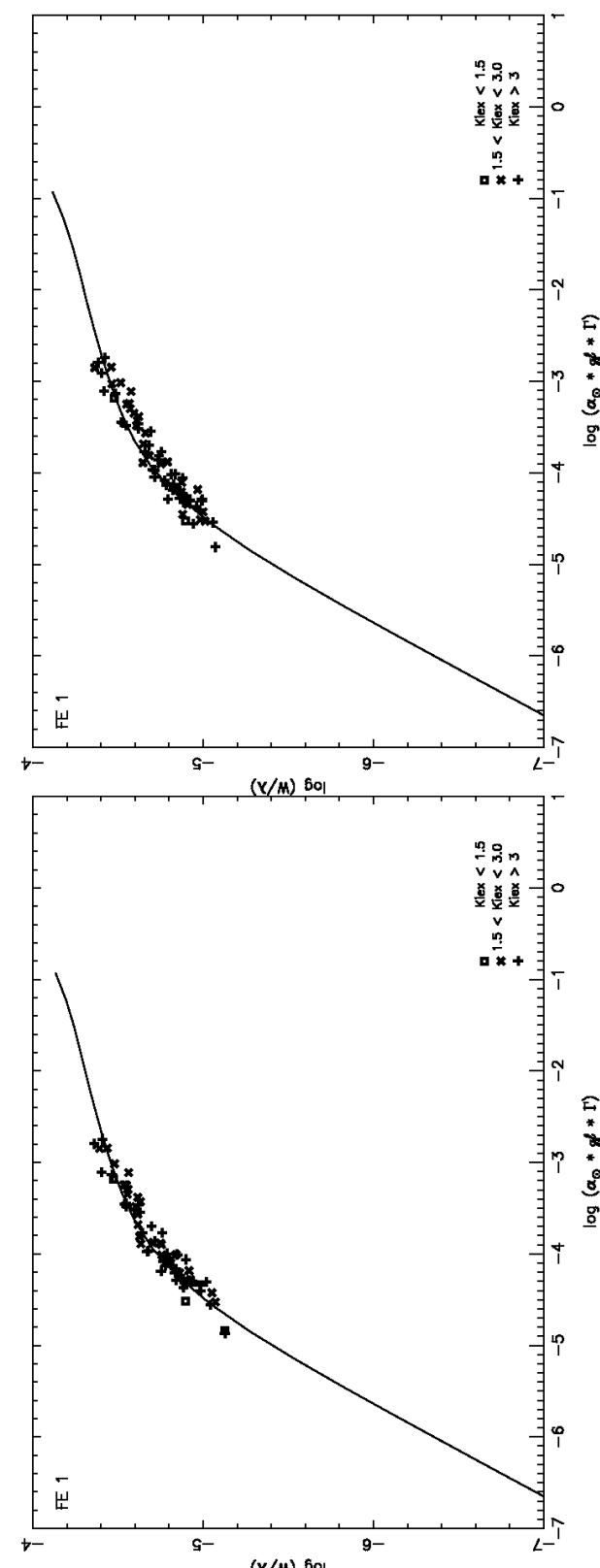

e

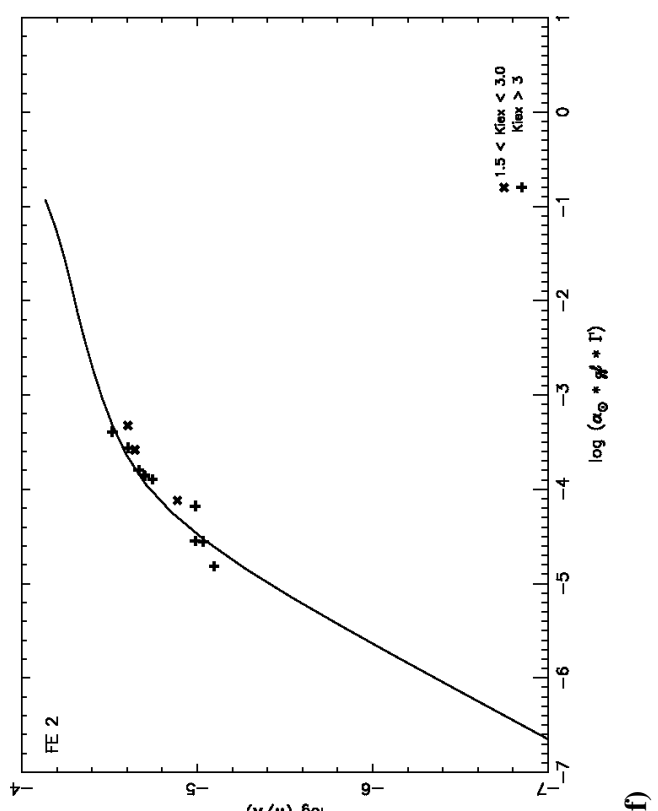

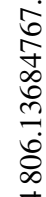

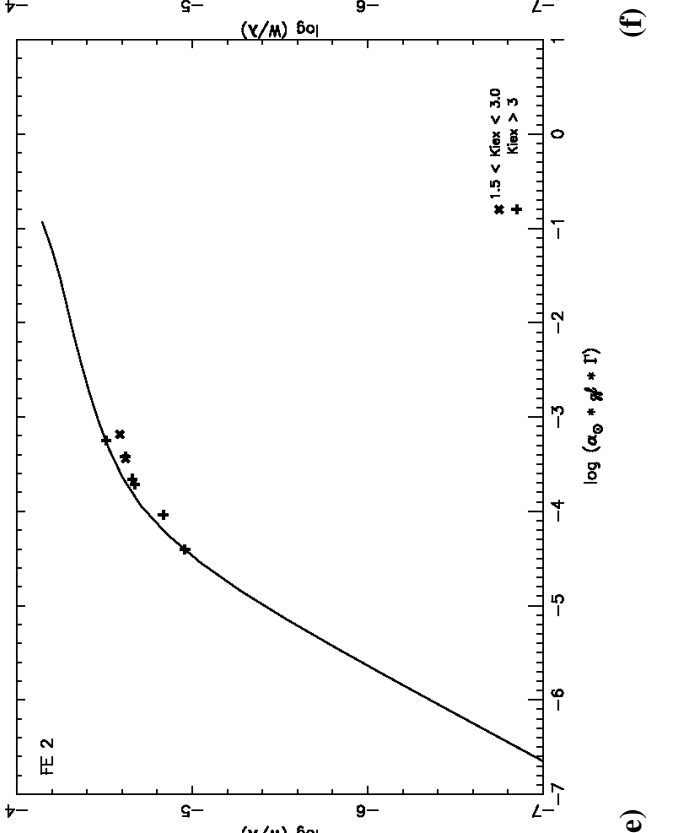

$\hat{e}$

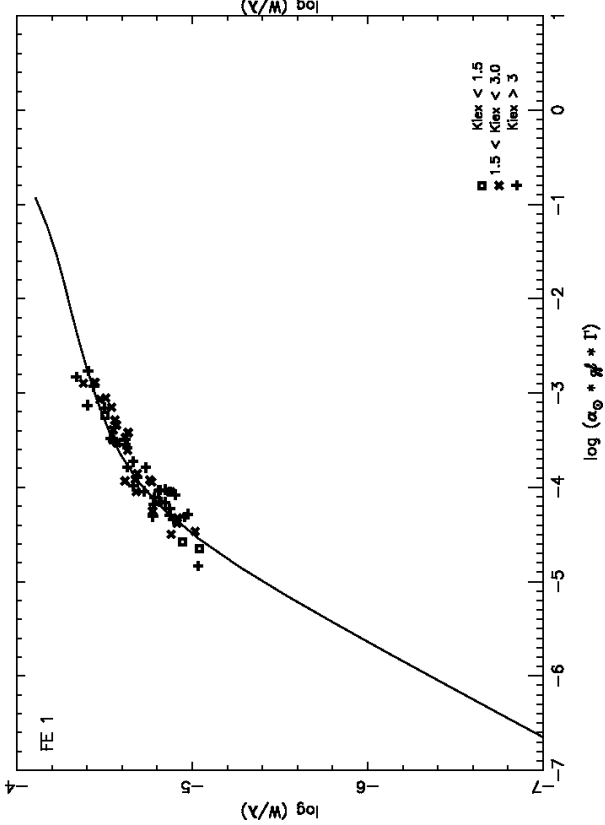

ङ

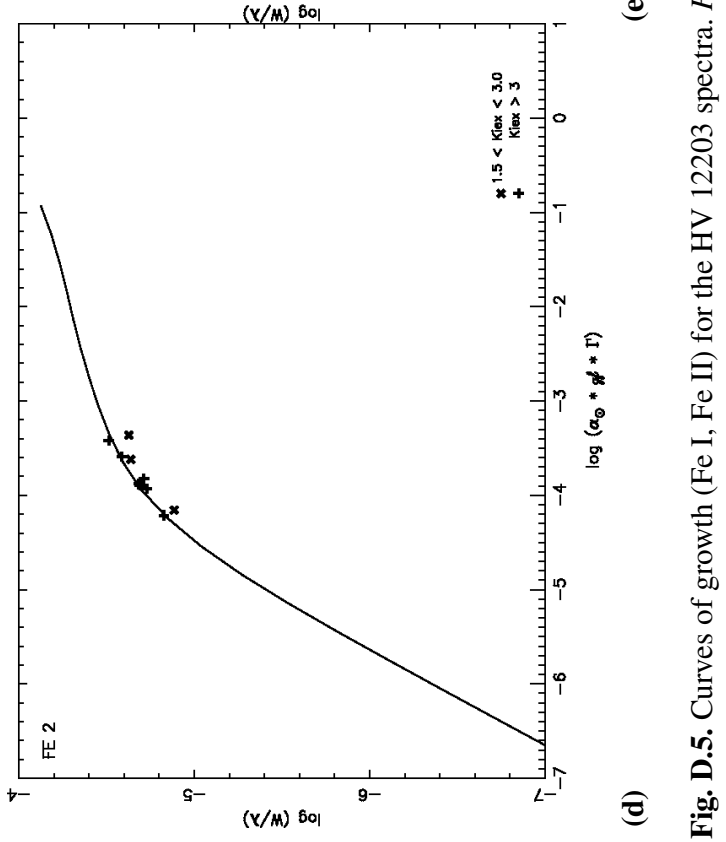

A85, page 31 of 36 
A\&A 608, A85 (2017)

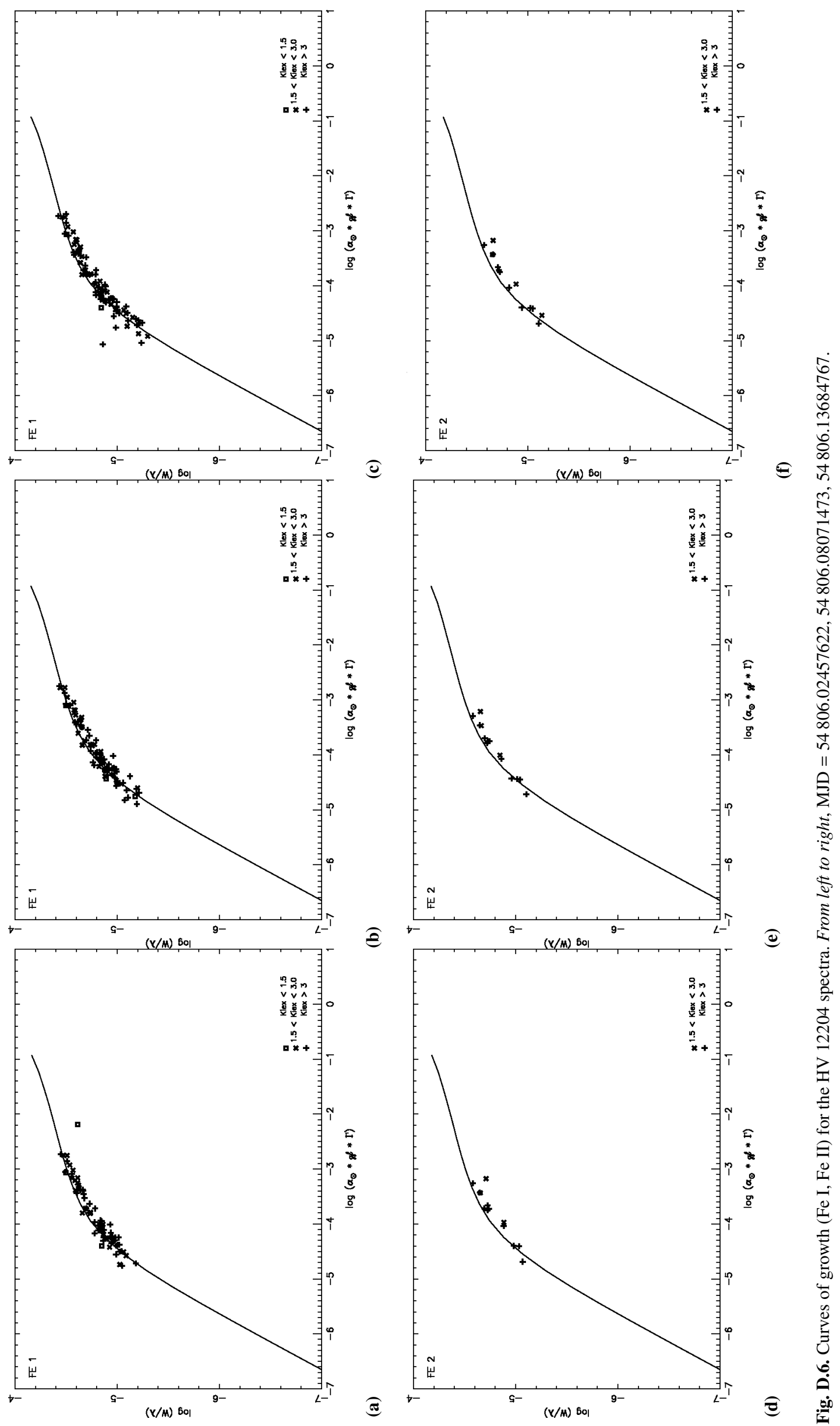


B. Lemasle et al.: Detailed chemical composition of classical Cepheids in the Magellanic Clouds
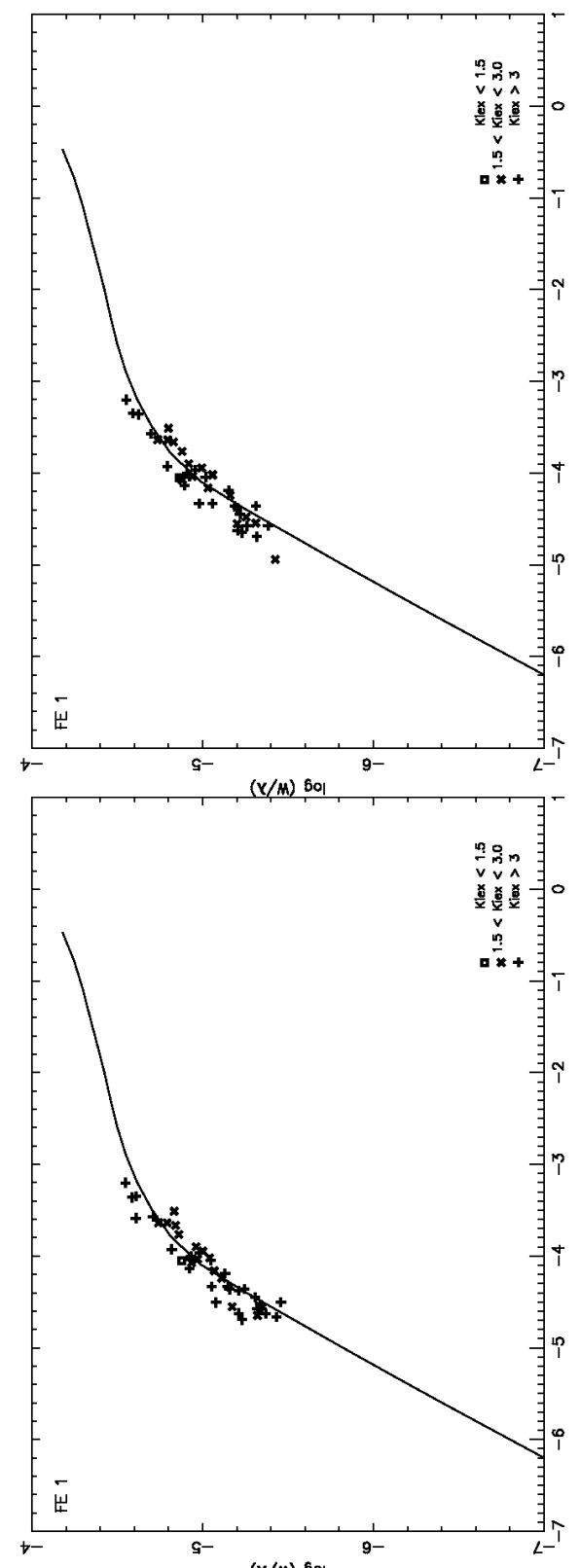

e

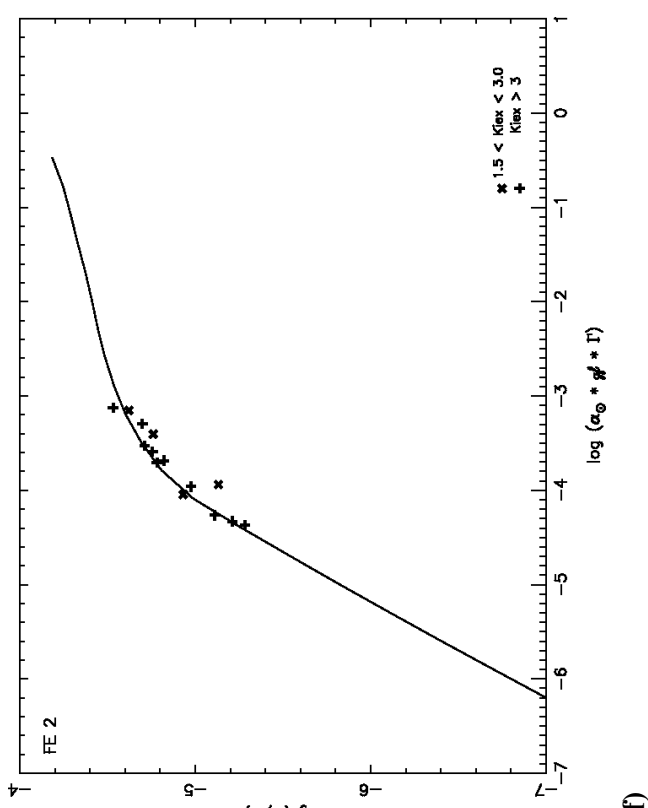

$\infty$
$n$
$n$
$n$
0
0
0
0
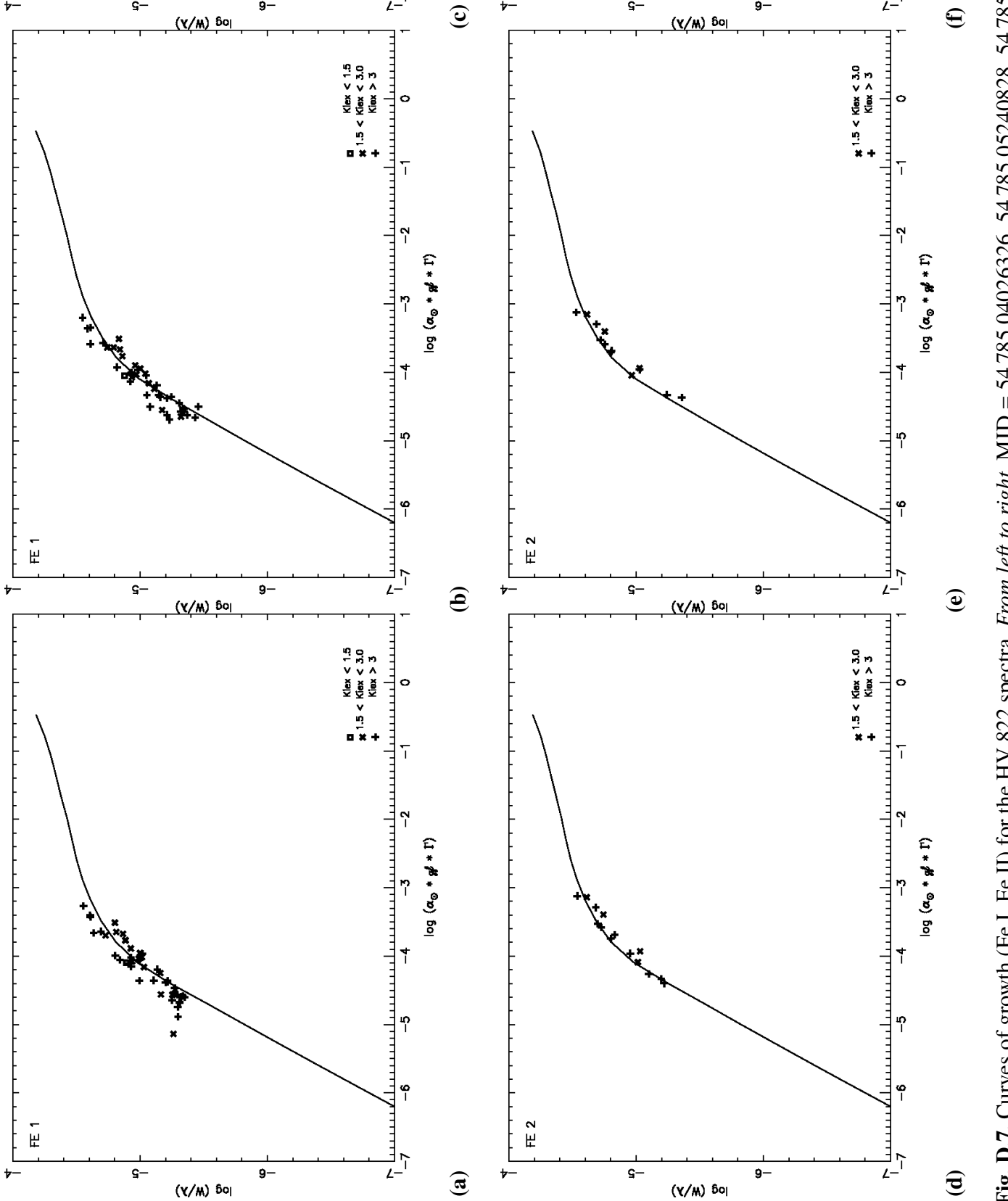

$\hat{\mathrm{e}}$

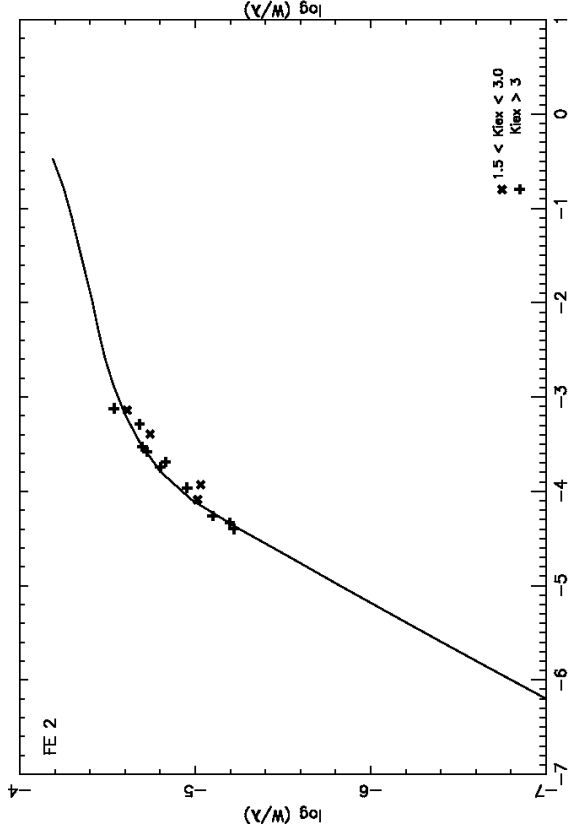


A\&A 608, A85 (2017)

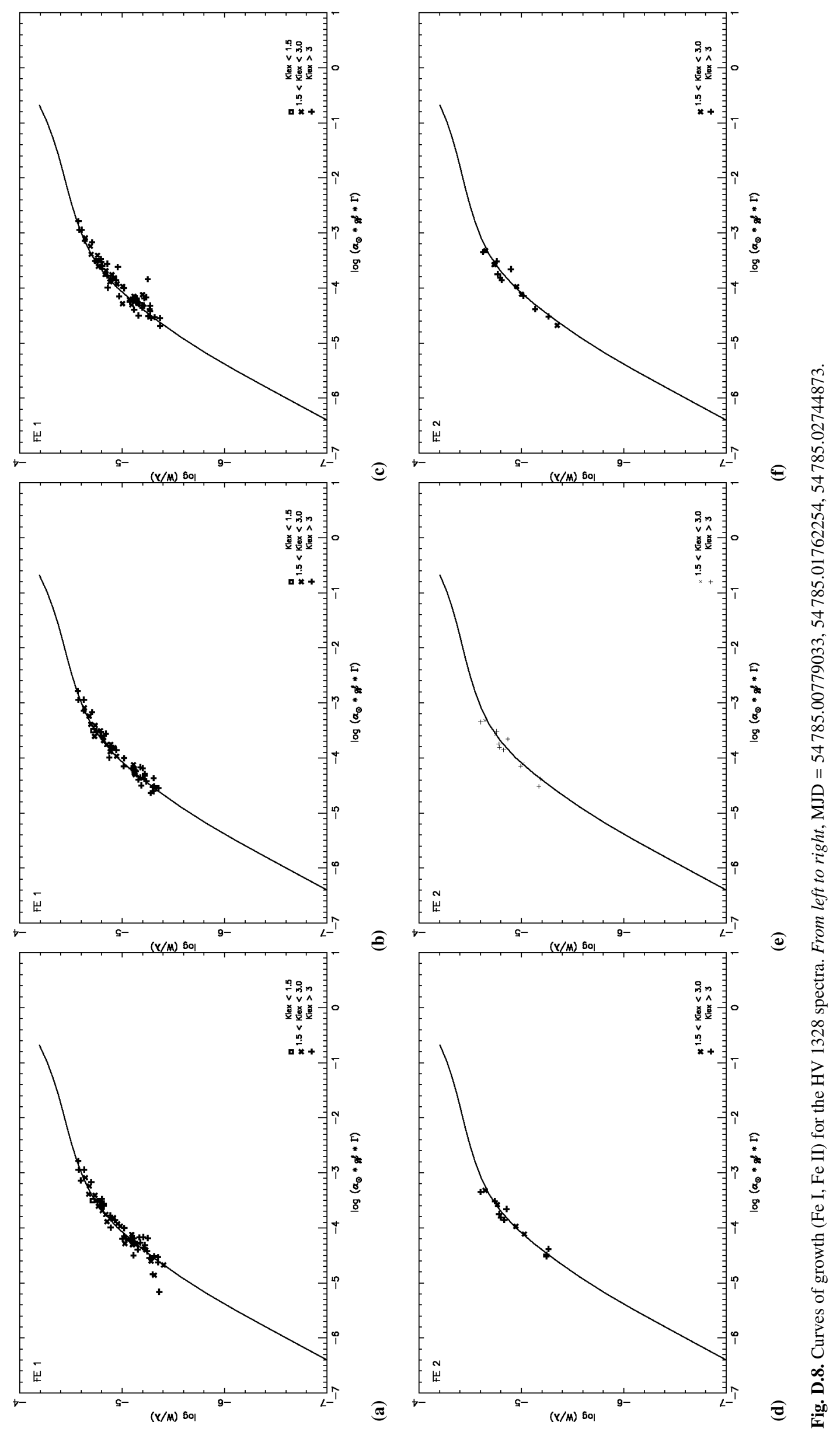


B. Lemasle et al.: Detailed chemical composition of classical Cepheids in the Magellanic Clouds
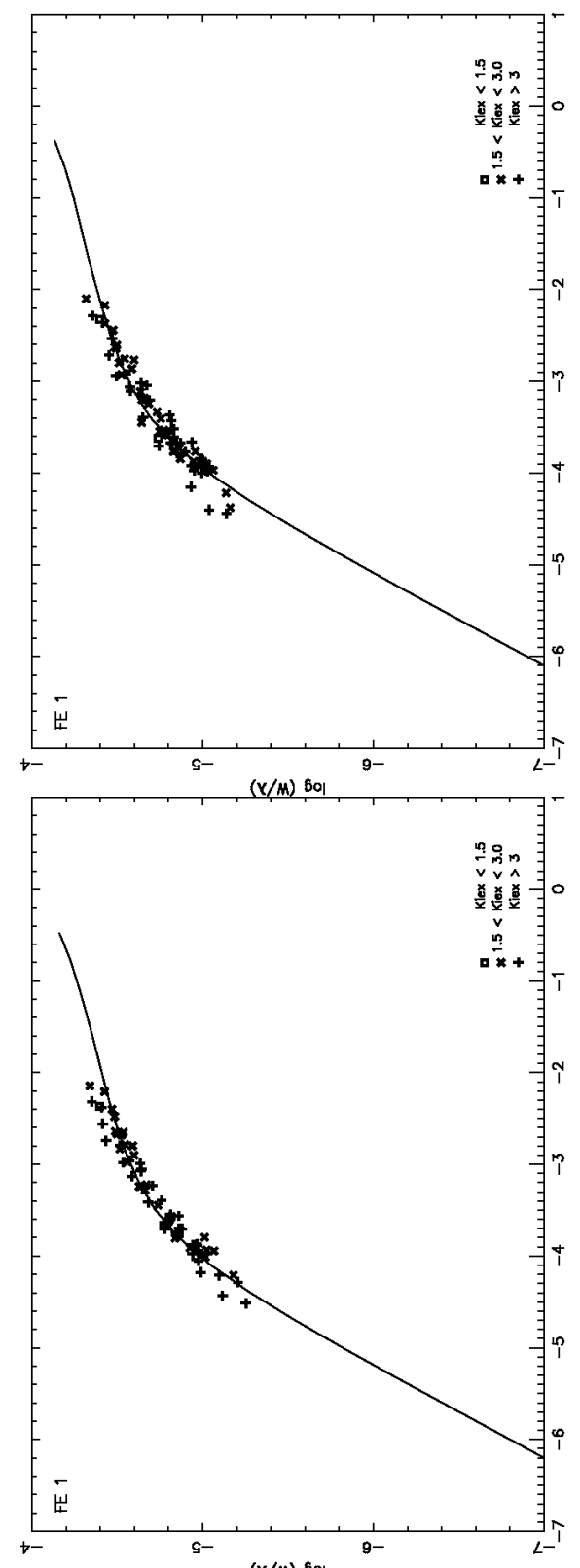

e

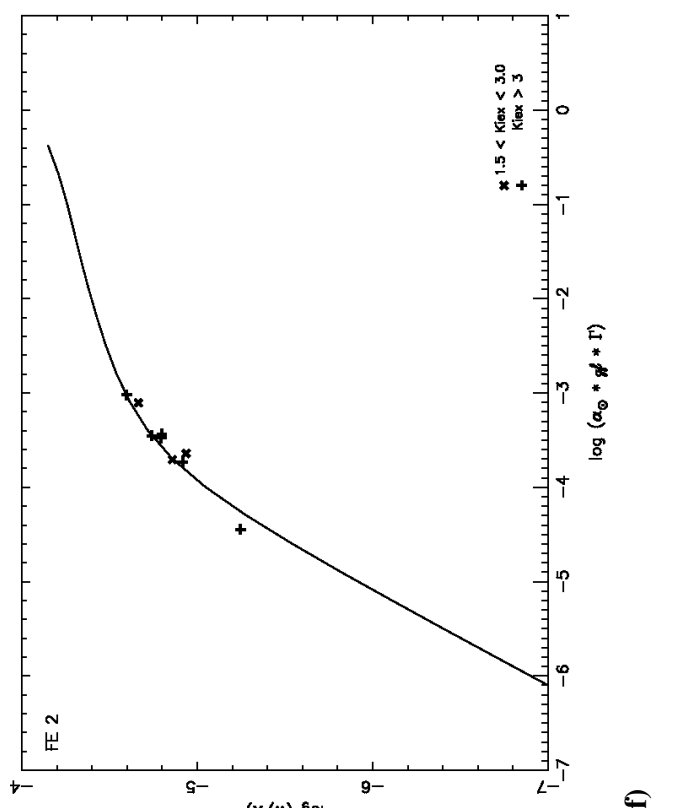

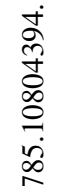
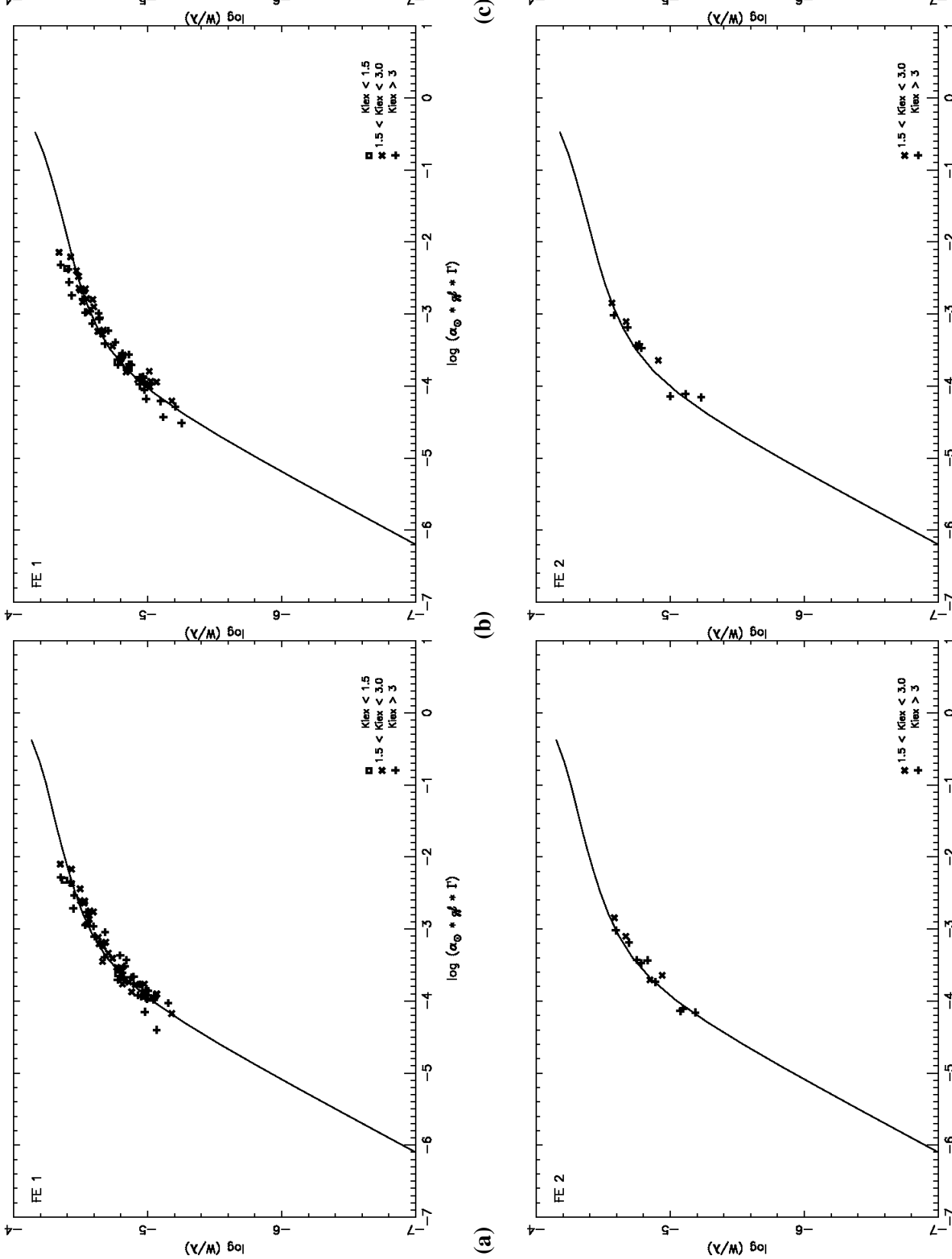

a

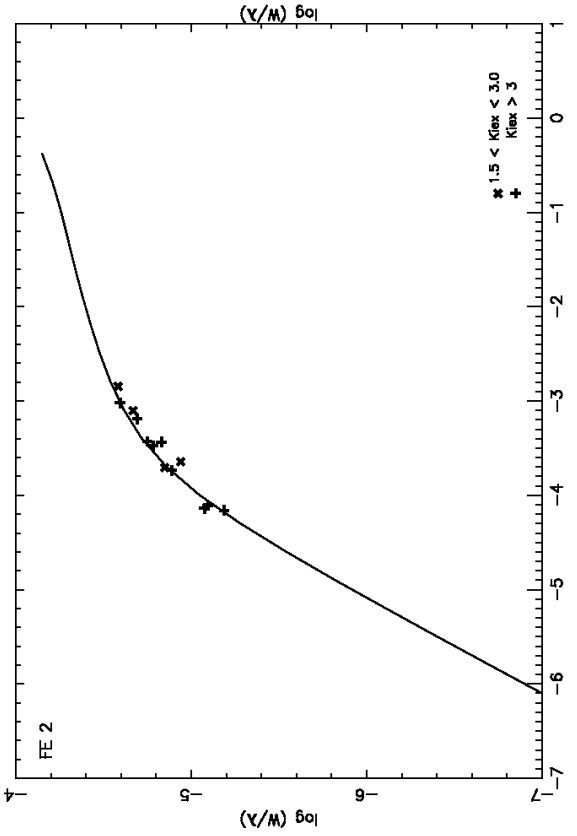

(2)

in

in 
A\&A 608, A85 (2017)
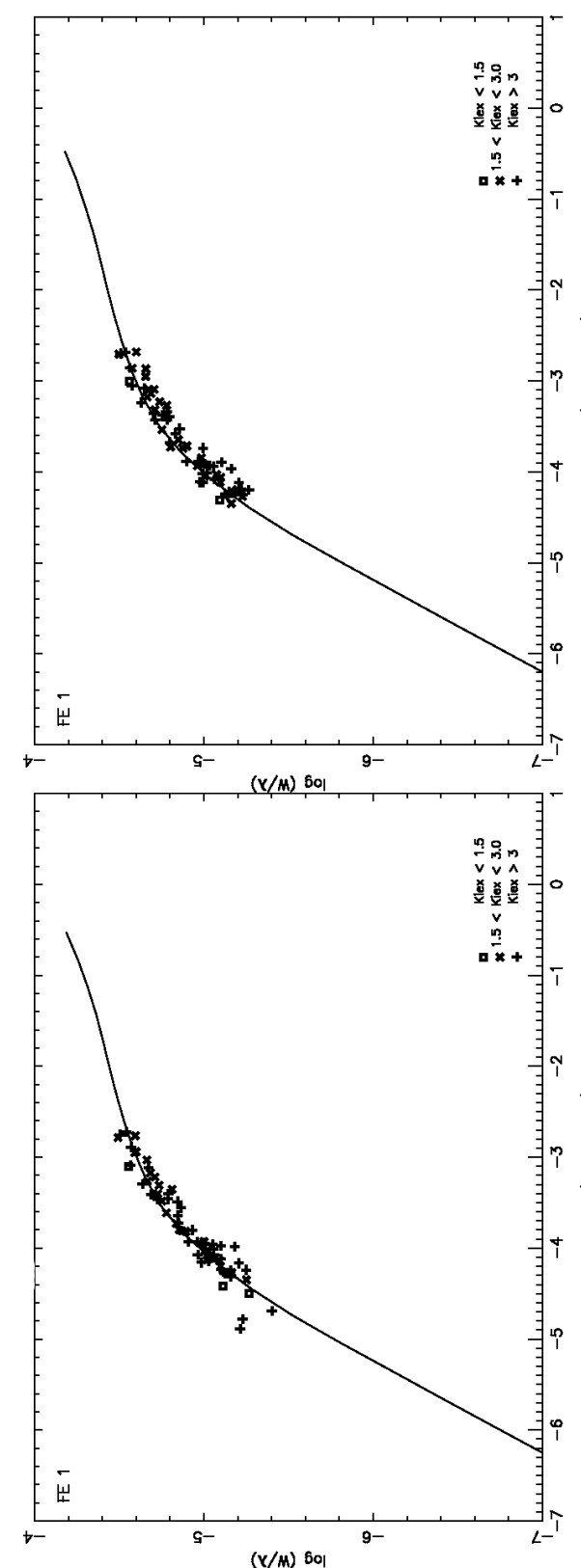

e

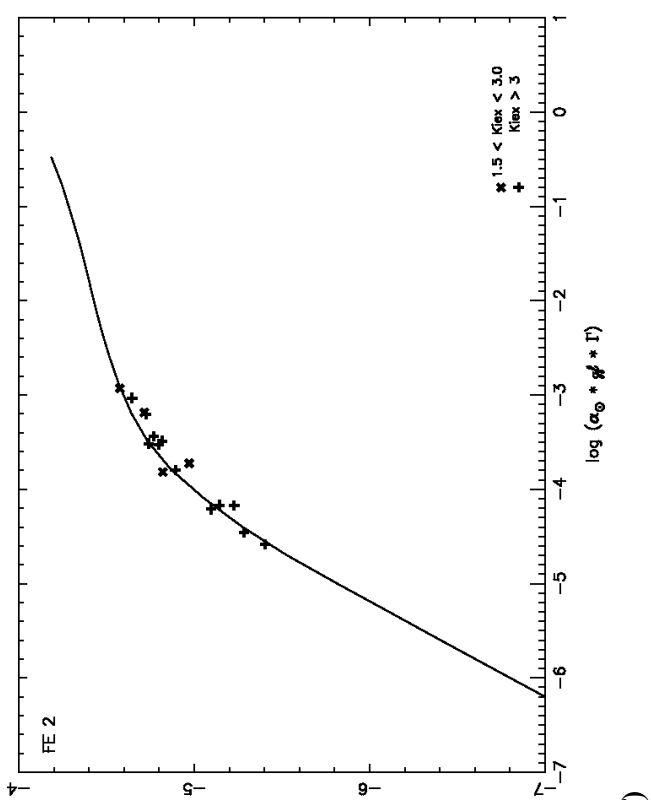

$\infty$
$\infty$
$\infty$
$\infty$
$\infty$
$\infty$
0
$n$
$\infty$
1
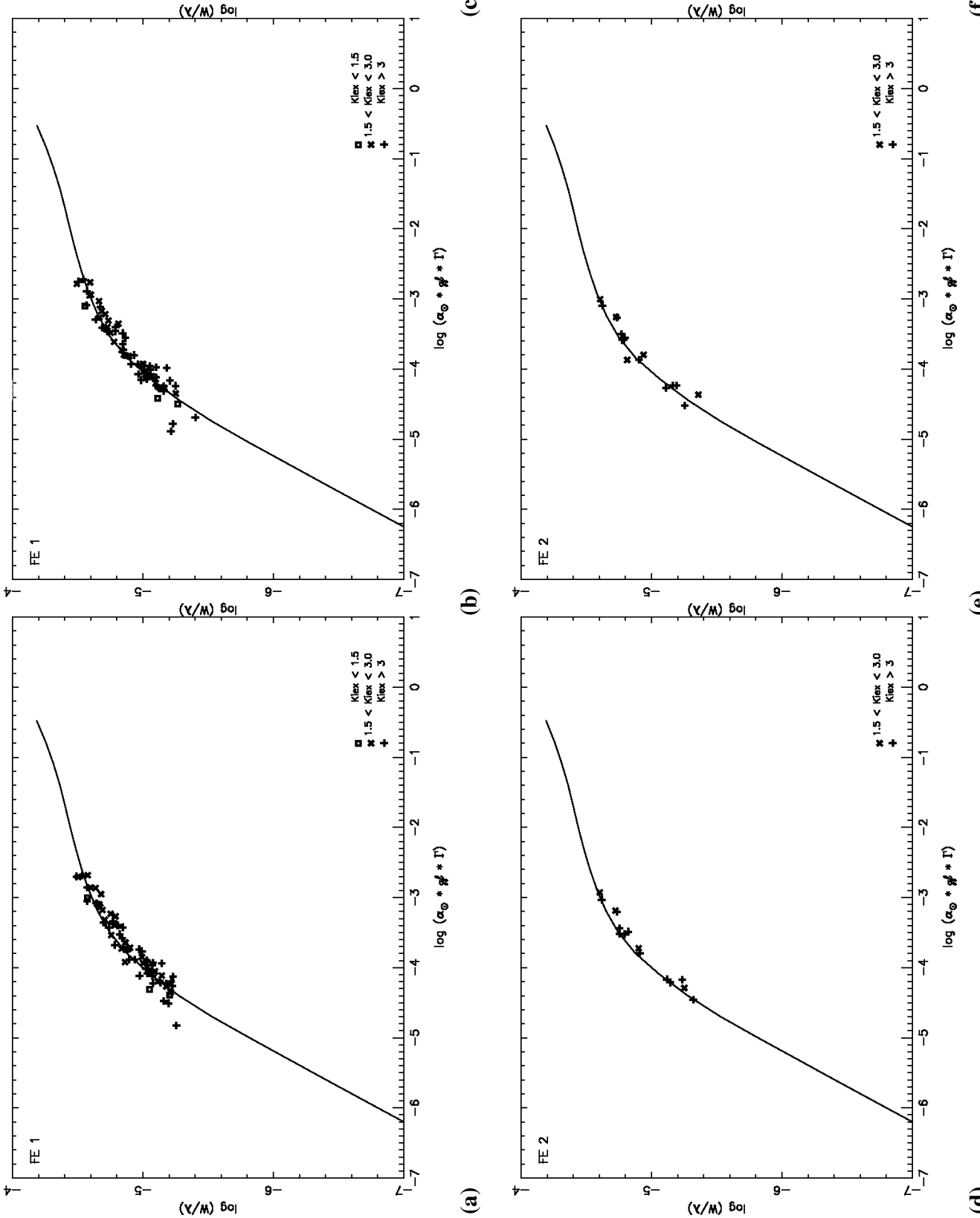

in

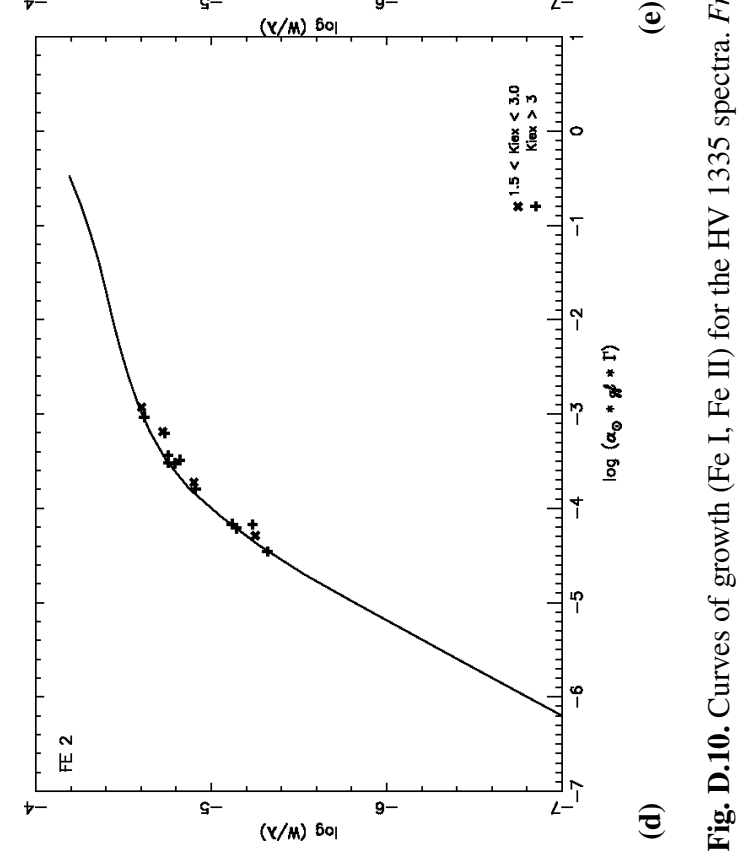

